\title{
INVARIANTS OF THE COX RINGS OF LOW-COMPLEXITY DOUBLE FLAG VARIETIES FOR CLASSICAL GROUPS
}

\author{
E. V. PONOMAREVA
}

\begin{abstract}
We find the algebras of unipotent invariants of Cox rings for all double flag varieties of complexity 0 and 1 for the classical groups; namely, we obtain presentations of these algebras. It is well known that such an algebra is simple in the case of complexity 0 . We show that, in the case of complexity 1 , the algebra in question is either a free algebra or a hypersurface. Knowing the structure of this algebra permits one to effectively decompose tensor products of irreducible representations into direct sums of irreducible representations.
\end{abstract}

\section{INTRODUCTION}

The problem of describing unipotent invariants of the Cox rings of double flag varieties is closely related to the problem of decomposing the tensor product of two irreducible representations of a semisimple complex algebraic group $G$ into a direct sum of irreducible representations. A geometric realization of irreducible representations is given by the Borel-Weil theorem, which states that every irreducible $G$-module can be realized as the space of sections of some $G$-line bundle $\mathcal{L}$ over the generalized flag variety $G / P$, where $P \subset G$ is a parabolic subgroup. The direct sum of spaces of sections of various line bundles over $G / P$ can be equipped with the structure of a graded ring, which is called the Cox ring and is denoted by $R(G / P)$. The tensor product of section spaces $H^{0}(G / P, \mathcal{L})$ and $H^{0}(G / Q, \mathcal{M})$ can be realized as the section space of the tensor product bundle $\mathcal{N}=\mathcal{L} \otimes \mathcal{M}$ over the double flag variety $X=G / P \times G / Q$. Thus, the sum of tensor products of representations realized in the spaces of sections of line bundles over $G / P$ and $G / Q$ is the Cox ring of the double flag variety. Knowing the structure of $R(X)$ or, more precisely, that of the algebra $R(X)^{U}$ of unipotent invariants (where $U \subset G$ is a maximal unipotent subgroup) permits one to decompose tensor products of representations into direct sums of irreducible representations. The algebra $R(X)^{U}$ is convenient to compute if one knows simple divisors on $X$ invariant under a Borel subgroup $B \subseteq G$. If $X$ is of complexity 0 or 1 , then the simple $B$-invariant divisors have a fairly simple structure.

The algebras of unipotent invariants for the Cox rings of double flag varieties of complexity 0 and 1 were found for the case of maximal parabolic subgroups by Littelmann [3] and Panyushev [4], respectively. In the present paper, we find the algebras of unipotent invariants for the Cox rings of all double flag varieties of complexity 0 and 1 for the classical groups. We also obtain a theorem on the structure of these algebras.

Let us briefly outline the structure of the paper. Section 2 introduces some notation and recalls some definitions and results. Section 3 deals with the structure of simple $B$-invariant divisors on varieties of complexity 0 and 1 . In Section 4 , we recall the definition of Cox rings and some results about them. We also explain how knowing the algebra $R(G / P \times G / Q)^{U}$ permits one to decompose tensor products of irreducible

2010 Mathematics Subject Classification. Primary 14L35; Secondary 14M17.

Key words and phrases. Double flag variety, Cox ring, complexity, linear representation, tensor product of representations, branching problem. 
representations realized in section spaces of line bundles over $G / P$ and $G / Q$. Section 5 presents theorems on the structure of algebras of unipotent invariants of Cox rings for low-complexity varieties. In Section 6, we discuss a method for computing these algebras of invariants. In the subsequent sections, we compute the generators and relations of these algebras for all double flag varieties of complexity 0 and 1 for the classical groups.

\section{MAin DEFinitions}

Let us present the main definitions, notation, and results to be used in the paper. After that, we explain what some of the definitions mean for the classical groups.

The ground field is the field of complex numbers, $G$ is a simple algebraic group, $B$ is a Borel subgroup containing a maximal torus $T, U \subseteq B$ is a maximal unipotent subgroup, and $P$ and $Q$ are parabolic subgroups.

The simple root system corresponding to the chosen Borel subgroup $B$ will be denoted by $\Pi$. We denote the simple roots by $\alpha_{i}$ (the numbering being in accordance with 1 , Table 1]) and the fundamental weights by $\omega_{i}$. The irreducible $G$-module with highest weight $\lambda$ will be denoted by $V(\lambda)$. Next, $V(\lambda)^{*}$ stands for the dual module and $\lambda^{*}$ for its highest weight. Thus, $V(\lambda)^{*} \simeq V\left(\lambda^{*}\right)$. The operation $*$ can be extended to all weights by linearity.

Let $X$ be a smooth irreducible algebraic $G$-variety.

Definition 1. The codimension of a generic $B$-orbit is called the complexity of the action of $G$ on $X$.

Varieties of complexity 0 are also said to be spherical.

By $H^{0}(X, \mathcal{L})$ we denote the space of sections of a line bundle $\mathcal{L}$ over $X$. Let $\mathcal{O}(D)$ be the line bundle corresponding to a divisor $D$. By $s_{D} \in H^{0}(X, \mathcal{O}(D))$ we denote the canonical section of $\mathcal{O}(D)$ satisfying the condition $\operatorname{div} s_{D}=D$. Here and in what follows, the word "divisor" is used to mean a Cartier (or locally principal) divisor. The section space $H^{0}(X, \mathcal{O}(D))$ can be identified with the set $\left\{f \in \mathbb{C}(X)^{\times} \mid \operatorname{div} f+D \geq 0\right\} \cup\{0\}$; this identification takes each function $f$ to the section $f \cdot s_{D}$.

The action of $G$ on $X$ lifts to an action on $\mathcal{L}$ linear on the fibers provided that $G$ is simply connected. By replacing $G$ with a simply connected covering, we can assume where necessary, without loss of generality, that $G$ is simply connected. If the divisor $D$ is $B$-invariant, then the canonical section $s_{D}$ is $B$-semi-invariant.

A parabolic subgroup $P \supseteq B$ can be specified by specifying a set of simple roots. To this end, let us decompose the parabolic subgroup into a semidirect product of a Levi subgroup by the unipotent radical, $P=L \curlywedge U_{P}$. The similar decomposition $\mathfrak{p}=\mathfrak{l} \oplus \mathfrak{u}_{P}$ holds for the tangent algebras. We say that the parabolic subgroup is defined by a set $I \subseteq \Pi$ of simple roots and denote it $P_{I}$ if the following decomposition holds for its tangent algebra:

$$
\mathfrak{p}_{I}=\mathfrak{t} \oplus \underset{\{\alpha>0\} \cup\{\alpha \in \mathbb{Z}(\Pi \backslash I)\}}{\mathfrak{g}_{\alpha},}
$$

where $\mathfrak{t}$ is the tangent algebra of the torus and $\mathfrak{g}_{\alpha}$ is the tangent algebra of the onedimensional unipotent subgroup corresponding to the root $\alpha$. Then $\mathfrak{l}$ and $\mathfrak{u}_{P}$ have the following decompositions:

$$
\mathfrak{l}=\mathfrak{t} \oplus \bigoplus_{\alpha \in \mathbb{Z}(\Pi \backslash I)} \mathfrak{g}_{\alpha}, \quad \mathfrak{u}_{P}=\bigoplus_{\{\alpha>0 \mid \alpha \notin \mathbb{Z}(\Pi \backslash I)\}} \mathfrak{g}_{\alpha} .
$$

With this definition, maximal parabolic subgroups correspond to subsets consisting of one simple root. 
By $P^{-}$we denote the negative parabolic subgroup containing the Borel subgroup $B^{-}$ and opposite to $P$. The tangent algebra $P_{I}^{-}$has the decomposition

$$
\mathfrak{p}_{I}=\mathfrak{t} \oplus \bigoplus_{\{\alpha<0\} \cup\{\alpha \in \mathbb{Z}(\Pi \backslash I)\}} \mathfrak{g}_{\alpha} .
$$

Recall that varieties of the form $G / P$ are called (partial) flag varieties.

Definition 2. A double flag variety is a variety of the form $G / P \times G / Q$, where $P$ and $Q$ are parabolic subgroups of $G$.

Now let us explain the above-introduced notions for the case of classical groups $(G=$ $\left.\mathrm{SL}_{n}, \mathrm{SO}_{n}, \mathrm{Sp}_{n}\right)$. We assume that $\mathrm{SO}_{n}$ preserves the bilinear form whose matrix has entries 1 on the secondary diagonal and zeros in all other positions, while $\mathrm{Sp}_{n}$ preserves the bilinear form whose matrix has entries 1 (starting from the upper right corner) followed by the same number of entries -1 on the secondary diagonal and zeros outside the secondary diagonal. Then $T$ is the subgroup of diagonal matrices, $B$ is the subgroup of upper triangular matrices, and $U$ is the subgroup of unipotent upper triangular matrices in $G$.

Let $\left\{e_{i}\right\}_{i=1, \ldots, n}$ be the standard basis in $\mathbb{C}^{n}$, and let $\varepsilon_{i}$ be the weight of $e_{i}$ with respect to the torus $T$. Then $\sum \varepsilon_{i}=0$ for $\mathrm{SL}_{n}$ and $\varepsilon_{k}=-\varepsilon_{n-k+1}$ for $\mathrm{Sp}_{n}$ and $\mathrm{SO}_{n}$. The expressions for $\alpha_{i}$ and $\omega_{i}$ via $\varepsilon_{i}$ are given in the following table.

\begin{tabular}{|c|l|l|}
\hline$G$ & \multicolumn{1}{|c|}{ Simple roots } & \multicolumn{1}{c|}{ Fundamental weights } \\
\hline $\mathrm{SL}_{n}$ & $\alpha_{i}=\varepsilon_{i}-\varepsilon_{i+1}, i<n$ & $\omega_{i}=\varepsilon_{1}+\cdots+\varepsilon_{i}, i<n$ \\
\hline $\mathrm{Sp}_{2 l}$ & $\alpha_{i}=\varepsilon_{i}-\varepsilon_{i+1}, i<l, \alpha_{l}=2 \varepsilon_{l}$ & $\omega_{i}=\varepsilon_{1}+\cdots+\varepsilon_{i}, i \leq l$ \\
\hline $\mathrm{SO}_{2 l+1}$ & $\alpha_{i}=\varepsilon_{i}-\varepsilon_{i+1}, i<l, \alpha_{l}=\varepsilon_{l}$ & $\omega_{i}=\varepsilon_{1}+\cdots+\varepsilon_{i}, i<l, \omega_{l}=\frac{1}{2}\left(\varepsilon_{1}+\cdots+\varepsilon_{l}\right)$ \\
\hline $\mathrm{SO}_{2 l}$ & $\alpha_{i}=\varepsilon_{i}-\varepsilon_{i+1}, i<l$, & $\omega_{i}=\varepsilon_{1}+\cdots+\varepsilon_{i}, i \leq l-2$, \\
& $\alpha_{l}=\varepsilon_{l-1}+\varepsilon_{l}$ & $\begin{array}{l}\omega_{l-1}=\frac{1}{2}\left(\varepsilon_{1}+\cdots+\varepsilon_{l-1}-\varepsilon_{l}\right), \\
\omega_{l}=\frac{1}{2}\left(\varepsilon_{1}+\cdots+\varepsilon_{l-1}+\varepsilon_{l}\right)\end{array}$ \\
& &
\end{tabular}

The operation $*$ acts identically on the weights for the groups $\mathrm{Sp}_{n}, \mathrm{SO}_{2 l+1}$, and $\mathrm{SO}_{4 m}$. One has $\varepsilon_{i}^{*}=-\varepsilon_{n-i+1}$ for $\mathrm{SL}_{n}$. Finally, $\varepsilon_{l}^{*}=-\varepsilon_{l}(l=2 m+1)$, and the operation $*$ acts identically on the remaining $\varepsilon_{i}$ for the group $\mathrm{SO}_{4 m+2}$.

For $G=\mathrm{SL}_{n}, \mathrm{Sp}_{n}, \mathrm{SO}_{2 l+1}$, the parabolic subgroups containing $B$ have a block upper triangular form. Hence the parabolic subgroups can be specified by the sizes of diagonal blocks; it will be convenient for us to start from the lower right block. There exists yet another class of parabolic subgroups for $G=\mathrm{SO}_{2 l}$; we will refer to the subgroups in this class as exceptional. These parabolic subgroups correspond to subsets $I$ such that $\alpha_{l-1} \in I$ and $\alpha_{l} \notin I$. The conjugation with the transposition of two middle basis vectors gives the subgroup $P_{I^{\prime}}$, where $I^{\prime}=I \cup\left\{\alpha_{l}\right\} \backslash\left\{\alpha_{l-1}\right\}$, which already has a block upper triangular form. The exceptional parabolic subgroup $P_{I}$ will be specified by the sizes of blocks of $P_{I^{\prime}}$ and will be indicated by a prime.

Let $I=\left\{\alpha_{i_{1}}, \ldots, \alpha_{i_{r}}\right\}, i_{1}<\cdots<i_{r}$. The following table describes the relationship between $I \subseteq \Pi$ and the sizes of blocks of $P_{I}$.

Note that the central block size is zero (i.e., the block is merely lacking) for $\mathrm{Sp}_{2 l}$ and $\mathrm{SO}_{2 l}$ if $i_{r}=l$. Note also that the subgroup of $\mathrm{SO}_{2 l}$ with central block of size 2 coincides with the subgroup with two central blocks of size 1 instead of the one central block.

The subgroup $P_{I}^{-}$is symmetric to $P_{I}$ with respect to the diagonal. 


\begin{tabular}{|c|c|}
\hline$G$ & \multicolumn{1}{|c|}{ Block sizes } \\
\hline $\mathrm{SL}_{n}$ & $\left(n-i_{r}, i_{r}-i_{r-1}, \ldots, i_{2}-i_{1}, i_{1}\right)$ \\
\hline $\mathrm{Sp}_{2 l}$ & $\left(i_{1}, i_{2}-i_{1}, \ldots, i_{r}-i_{r-1}, 2\left(l-i_{r}\right), i_{r}-i_{r-1}, \ldots, i_{2}-i_{1}, i_{1}\right)$ \\
\hline $\mathrm{SO}_{2 l+1}$ & $\left(i_{1}, i_{2}-i_{1}, \ldots, i_{r}-i_{r-1}, 2\left(l-i_{r}\right)+1, i_{r}-i_{r-1}, \ldots, i_{2}-i_{1}, i_{1}\right)$ \\
\hline $\mathrm{SO}_{2 l}$ & $\begin{array}{l}\left(i_{1}, i_{2}-i_{1}, \ldots, i_{r}-i_{r-1}, 2\left(l-i_{r}\right), i_{r}-i_{r-1}, \ldots, i_{2}-i_{1}, i_{1}\right) \text { if } \alpha_{l-1} \notin I \text { or } \alpha_{l} \in I, \\
\left(i_{1}, i_{2}-i_{1}, \ldots, l-i_{r-1}, l-i_{r-1}, \ldots, i_{2}-i_{1}, i_{1}\right)^{\prime} \text { if } \alpha_{l-1} \in I \text { and } \alpha_{l} \notin I\end{array}$ \\
\hline
\end{tabular}

Let $P$ be specified by the block sizes $\left(k_{1}, \ldots, k_{s}\right)$ of $\left(k_{1}, \ldots, k_{s}\right)^{\prime}$. For technical reasons, we prefer considering the flag variety corresponding to the negative parabolic subgroup $P^{-}$. The points of the flag variety $G / P^{-}$are flags of nested subspaces of dimensions $k_{1}, k_{1}+k_{2}, \ldots$ in $\mathbb{C}^{n}$. For $G=\mathrm{SO}_{n}, \mathrm{Sp}_{n}$, in addition, the subspaces should be isotropic or coisotropic, and if a flag contains a subspace $W$, then the flag should also contain the subspace $W^{\perp}$. If the subgroup is not exceptional, then the subspaces in a flag in $G / P^{-}$will be denoted by $R_{i}$, where the subscript stands for dimension. If the subgroup is exceptional, then the subspace of dimension $l$ will be denoted by $R_{l}^{\prime}$ and the subspaces of other dimensions will be denoted in the same way as above.

For the double flag varieties $X=G / P^{-} \times G / Q^{-}$, the subspaces in the flags corresponding to points in $G / Q^{-}$will be denoted in a similar way by $S_{j}$ or $S_{l}^{\prime}$.

As was mentioned in the Introduction, we are interested in double flag varieties of complexity 0 and 1. Such varieties were classified by Littelmann [3], Stembridge [6], Panyushev 44, and the author [5. The list of double flag varieties of complexity $\leq 1$ for the classical groups is given in Table 1. The corresponding pairs of parabolic subgroups are specified by the sizes of the respective diagonal blocks (starting from the lower right) with prime added in the case of an exceptional subgroup. The classification is up to a permutation of subgroups; for the group $\mathrm{SL}_{n}$, also up to a diagram automorphism (i.e., simultaneous transposition with respect to the secondary diagonal); for the group $\mathrm{SO}_{2 l}$, also up to a diagram automorphism transposing the "whiskers" of the Dynkin diagram (i.e., simultaneous conjugation with the transposition of two middle basis vectors).

\section{Geometry of LOW-COMPlexity varieties}

In this section, we discuss the structure of $B$-invariant divisors on varieties of complexity 0 and 1. More details on the structure of divisors can be found, e.g., in [7, $16.2]$.

There are finitely many simple $B$-invariant divisors on a spherical variety, because they are components of the complement of an open $B$-orbit.

Now let $X$ be a rational variety of complexity 1 . By the Lüroth theorem, $\mathbb{C}(X)^{B} \simeq$ $\mathbb{C}\left(\mathbb{P}^{1}\right)$. The field embedding $\mathbb{C}(X) \supseteq \mathbb{C}(X)^{B}$ determined a rational mapping $X \rightarrow \mathbb{P}^{1}$ whose generic fibers are the closures of generic $B$-orbits. Hence the simple $B$-invariant divisors have the following structure: all but finitely many of them form a family. The divisors in this family will be referred to as parametric, and the remaining divisors, which are finitely many, as exceptional.

By $\operatorname{ord}_{D}(f)$ we denote the order of a function $f$ along a simple divisor $D$. By restricting $\operatorname{ord}_{D}$ to $\mathbb{C}(X)^{B} \simeq \mathbb{C}\left(\mathbb{P}^{1}\right)$, we obtain a valuation on the field $\mathbb{C}\left(\mathbb{P}^{1}\right)$ with some center $z_{D} \in \mathbb{P}^{1}$ and order $h_{D} \in \mathbb{Z}_{+}$of the local coordinate in $z_{D}$. (For $h_{D}=0$, one can take any point of $\mathbb{P}^{1}$ for $z_{D}$.). The divisors $D$ with $h_{D}>0$ are taken to $z_{D}$ by the mapping $X \rightarrow \mathbb{P}^{1}$. The remaining divisors are mapped onto $\mathbb{P}^{1}$ dominantly. The parametric 
TABLE 1. Double flag varieties of complexity 0 and 1 for the classical groups

\begin{tabular}{|c|c|c|c|c|}
\hline & \multicolumn{2}{|c|}{ Complexity 0} & \multicolumn{2}{|c|}{ Complexity 1} \\
\hline$G$ & $P$ & $Q$ & $P$ & $Q$ \\
\hline $\mathrm{SL}_{n}$ & $\begin{array}{l}\left(p_{1}, p_{2}\right) \\
\left(p_{1}, p_{2}\right) \\
\left(p_{1}, p_{2}\right) \\
(2, p) \\
(1, p)\end{array}$ & $\begin{array}{l}\left(q_{1}, q_{2}\right) \\
\left(1, q_{2}, q_{3}\right) \\
\left(q_{1}, 1, q_{3}\right) \\
\left(q_{1}, q_{2}, q_{3}\right) \\
\left(q_{1}, q_{2}, \ldots, q_{s}\right)\end{array}$ & $\begin{array}{l}(2, p), p \geq 2 \\
\left(p_{1}, p_{2}\right), p_{1}, p_{2} \geq 2 \\
\left(p_{1}, p_{2}\right), p_{1}, p_{2} \geq 2 \\
(3, p), p \geq 3 \\
\left(p_{1}, p_{2}\right), p_{1}, p_{2} \geq 3 \\
\left(p_{1}, p_{2}\right), p_{1}, p_{2} \geq 3 \\
\left(p_{1}, p_{2}, p_{3}\right) \\
\left(p_{1}, p_{2}, p_{3}\right)\end{array}$ & $\begin{array}{l}\left(q_{1}, q_{2}, q_{3}, q_{4}\right) \\
(1,1,1, q) \\
(1,1, q, 1) \\
\left(q_{1}, q_{2}, q_{3}\right), q_{1}, q_{2}, q_{3} \geq 2 \\
(2,2, q), q \geq 2 \\
(2, q, 2), q \geq 2 \\
(1,1, q) \\
(1, q, 1)\end{array}$ \\
\hline $\mathrm{Sp}_{n}$ & $\begin{array}{l}(p, p) \\
(p, p) \\
(1, p, 1)\end{array}$ & $\begin{array}{l}(p, p) \\
(1, q, 1) \\
\left(q_{1}, q_{2}, q_{1}\right)\end{array}$ & $\begin{array}{l}(p, p) \\
(1, p, 1) \\
(1, p, 1) \\
(2,2)\end{array}$ & $\begin{array}{l}(2, q, 2) \\
\left(q_{1}, q_{2}, q_{2}, q_{1}\right) \\
\left(q_{1}, q_{2}, q_{3}, q_{2}, q_{1}\right) \\
(1,1,1,1)\end{array}$ \\
\hline $\mathrm{SO}_{n}$ & $\begin{array}{l}(p, p) \\
(p, p) \\
(p, p) \\
(p, p) \\
(p, p) \\
(p, p) \\
(p, p) \\
(p, 1, p) \\
(1, p, 1) \\
(1, p, 1) \\
(4,4)\end{array}$ & $\begin{array}{l}(p, p) \\
(p, p)^{\prime} \\
\left(q_{1}, q_{2}, q_{1}\right), q_{1} \leq 3 \\
(q, 2, q) \\
(1, q, q, 1) \\
(1, q, q, 1)^{\prime} \\
(1,1, q, 1,1) \\
(p, 1, p) \\
\left(q_{1}, q_{2}, q_{1}\right) \\
\left(q_{1}, q_{2}, q_{2}, q_{1}\right) \\
(2,2,2,2)^{\prime}\end{array}$ & $\begin{array}{l}(2, p, 2), p \geq 2 \\
(1, p, 1) \\
(1, p, 1) \\
(4,4) \\
(4,4) \\
(4,4) \\
(4,4) \\
(4,4) \\
(5,5) \\
(5,5) \\
(5,5) \\
(5,5) \\
(6,6) \\
(2,2,2) \\
(2,2,2) \\
(2,1,2) \\
(1,2,2,1) \\
(1,2,2,1)\end{array}$ & $\begin{array}{l}(q, 1, q) \\
\left(q_{1}, q_{2}, q_{3}, q_{2}, q_{1}\right) \\
\left(q_{1}, q_{2}, q_{3}, q_{3}, q_{2}, q_{1}\right) \\
(2,2,2,2) \\
(1,2,2,2,1) \\
(2,1,2,1,2) \\
(1,1,2,2,1,1) \\
(1,1,2,2,1,1)^{\prime} \\
(2,3,3,2) \\
(2,3,3,2)^{\prime} \\
(3,2,2,3) \\
(3,2,2,3)^{\prime} \\
(4,4,4) \\
(2,2,2) \\
(1,2,2,1) \\
(1,1,1,1,1) \\
(1,2,2,1) \\
(1,2,2,1)^{\prime}\end{array}$ \\
\hline
\end{tabular}

divisors are characterized by the fact that $h_{D}=1$ and there does not exist a different $B$-invariant simple divisor $D^{\prime}$ with $z_{D}=z_{D^{\prime}}$ and $h_{D^{\prime}}>0$. The points $z$ on the projective line for which there does not exist a parametric divisor $D$ with $z_{D}=z$ are said to be 
exceptional. There exist finitely many exceptional points. The other points of $\mathbb{P}^{1}$ are said to be ordinary.

For the rational mapping $X \rightarrow \mathbb{P}^{1}$ considered above, there exist sections $F$ and $F^{\prime}$ of some $G$-line bundle $\mathcal{L} \rightarrow X$ such that the mapping is given by the formula $x \mapsto(F(x)$ : $F^{\prime}(x)$ ). Since the mapping is constant on the $B$-orbits, it follows that these sections are $B$-semi-invariant of one and the same weight. Thus, we have determined the mapping with the use of the linear divisor system $\left\{D \mid D=\operatorname{div} s, s \in\left\langle F, F^{\prime}\right\rangle\right\}$. The parametric divisors are exactly the simple divisors of the linear system. For an ordinary point $z=(p: q) \in \mathbb{P}^{1}$, the parametric divisor $D$ with $z_{D}=z$ has the form $D=\operatorname{div}\left(q F-p F^{\prime}\right)$. For an exceptional point $z=(p: q) \in \mathbb{P}^{1}$, the $\operatorname{divisor} D=\operatorname{div}\left(q F-p F^{\prime}\right)$ is not simple, and one has the following decomposition:

$$
\operatorname{div}\left(q F-p F^{\prime}\right)=\sum_{z_{D_{i}}=z} h_{D_{i}} D_{i}
$$

where the $D_{i}$ are all simple exceptional divisors lying in the preimage of $z$.

\section{Cox Rings AND TEnsor PRODUCTS OF MODUleS}

Now let us describe a method for decomposing a tensor product of irreducible $G$ modules in terms of $U$-invariants of the Cox rings of double flag varieties.

Consider the space $R(X)=\bigoplus H^{0}(X, \mathcal{L})$, where the sum is over pairwise nonisomorphic line bundles $\mathcal{L}$. We wish to equip $R(X)$ with the structure of a ring. Assume that the Picard group $\operatorname{Pic}(X)$ is free. Then $R(X)$ is a ring with respect to the tensor multiplication of sections and is naturally graded by the Picard group. The resulting ring is called the Cox ring of the variety $X$. The grading determines the multidegree of homogeneous elements, and the sections of line bundles are exactly the multihomogeneous elements of $R(X)$. A more detailed definition of the Cox ring can be found, e.g., in [2].

First, we describe the Picard group of a flag variety. Let $P=P_{\left\{\alpha_{i}\right\}}$ be a maximal parabolic subgroup. The variety $G / P^{-}$is embedded in $\mathbb{P}\left(V\left(\omega_{i}^{*}\right)\right)$ as the projectivization of the orbit of the lowest vector. The highest vector $f_{\omega_{i}} \in V\left(\omega_{i}^{*}\right)^{*} \simeq V\left(\omega_{i}\right)$ of the dual module is a linear form on $\mathbb{P}\left(V\left(\omega_{i}^{*}\right)\right)$ and determines a $P$-invariant simple divisor on $G / P^{-}$, which is called a Schubert divisor. We denote it by $\Delta_{P, i}$. The Picard group of the variety $G / P^{-}$is generated by the class of the divisor $\Delta_{P, i}$. The canonical section $s_{\Delta_{P, i}}$ is the restriction to $G / P^{-}$of the linear form $f_{\omega_{i}}$; its weight is equal to $\omega_{i}$.

Now let $X=G / P_{I}^{-}, I=\left\{\alpha_{i_{1}}, \ldots, \alpha_{i_{r}}\right\}$. Let $\alpha \in I$. There exists a $G$-equivariant surjective morphism $G / P_{I}^{-} \rightarrow G / P_{\{\alpha\}}^{-}$. The preimages of Schubert divisors on $G / P_{\{\alpha\}}^{-}$ under such morphisms (for various $\alpha \in I$ ) are $P_{I}$-invariant divisors on $G / P_{I}^{-}$, which will also be called Schubert divisors and denoted in the same way. The Schubert divisors are components of the complement of an open $B$-orbit and exhaust all simple $B$-invariant divisors on $G / P_{I}^{-}$. The Picard group is freely generated by the classes of Schubert divisors. The weights of the canonical solutions corresponding to Schubert divisors are equal to $\omega_{i_{1}}, \ldots, \omega_{i_{r}}$.

Now we can find the Cox ring of the flag variety. Since $\operatorname{Pic}\left(G / P_{I}^{-}\right) \simeq \mathbb{Z}^{r}$, we see that the multidegree of a section is given by an $r$-vector. Let

$$
D=\sum m_{i_{k}} \Delta_{P, i_{k}}, \quad \lambda=\sum m_{i_{k}} \omega_{i_{k}} .
$$

Then the sections in $H^{0}(X, \mathcal{O}(D))$ have multidegree $\left(m_{i_{1}}, \ldots, m_{i_{r}}\right)$ in the ring $R\left(G / P_{I}^{-}\right)$. In view of the isomorphism $H^{0}(X, \mathcal{O}(D)) \simeq V(\lambda)$, we obtain

$$
R\left(G / P_{I}^{-}\right) \simeq \underset{\substack{\lambda=m_{i_{1}} \omega_{i_{1}}+\cdots+m_{i_{r}} \omega_{i_{r}} \\ m_{i_{1}}, \ldots, m_{i_{r}} \geq 0}}{\bigoplus} V(\lambda) .
$$


Now consider the double flag variety $X=G / P_{I}^{-} \times G / P_{J}^{-}$, where $I=\left\{\alpha_{i_{1}}, \ldots, \alpha_{i_{r}}\right\}$ and $J=\left\{\alpha_{j_{1}}, \ldots, \alpha_{j_{t}}\right\}$. The Picard group is freely generated by the preimages of Schubert divisors on $G / P_{I}^{-}$and $G / P_{J}^{-}$under the canonical projections $X \rightarrow G / P_{I}^{-}$and $X \rightarrow$ $G / P_{J}^{-}$, respectively. By abuse of speech, such preimages of Schubert divisors will be called Schubert divisors as well. The Cox ring of the double flag variety can be represented in the form

$$
R(X)=R\left(G / P_{I}^{-}\right) \otimes R\left(G / P_{J}^{-}\right) \simeq \bigoplus_{\substack{\lambda=m_{i_{1}} \omega_{i_{1}}+\cdots+m_{i_{r}} \omega_{i_{r}}, m_{i_{1}}, \ldots, m_{i_{r}} \geq 0 \\ \mu=n_{j_{1}} \omega_{j_{1}}+\cdots+n_{j_{t}} \omega_{j_{t}}, n_{j_{1}}, \ldots, n_{j_{t}} \geq 0}} V(\lambda) \otimes V(\mu) .
$$

The multidegree is given by an $(r+t)$-vector. Let

$$
\lambda=\sum m_{i_{k}} \omega_{i_{k}}, \quad \mu=\sum n_{j_{l}} \omega_{j_{l}} .
$$

Then the submodule $V(\lambda) \otimes V(\mu) \subset R(X)$ consists exactly of multihomogeneous elements of multidegree

$$
\left(m_{i_{1}}, \ldots, m_{i_{r}}, n_{j_{1}}, \ldots, n_{j_{t}}\right)=:(\bar{m}, \bar{n}) .
$$

The multiplicity of $V(\nu)$ in $V(\lambda) \otimes V(\mu)$ is equal to the dimension of the space $R(X)_{(\bar{m}, \bar{n}), \nu}^{U}$, where $R(X)_{(\bar{m}, \bar{n}), \nu}^{U}$ is the space of $U$-invariants of $B$-weight $\nu$ and multidegree $(\bar{m}, \bar{n})$. These dimensions can readily be computed for the following cases.

Theorem 1. Let $R(X)^{U}$ be free, and let the elements of its minimal system of homogeneous generators have weights $\nu_{1}, \ldots, \nu_{d}$ and multidegrees $\left(\bar{m}_{1}, \bar{n}_{1}\right), \ldots,\left(\bar{m}_{d}, \bar{n}_{d}\right)$. Then the following decomposition holds:

$$
V(\lambda) \otimes V(\mu) \simeq \bigoplus_{k_{1}\left(\bar{m}_{1}, \bar{n}_{1}\right)+\cdots+k_{d}\left(\bar{m}_{d}, \bar{n}_{d}\right)=(\bar{m}, \bar{n})} V\left(k_{1} \nu_{1}+\cdots+k_{d} \nu_{d}\right) .
$$

Proof. The assertion obviously follows from the preceding.

Theorem 2. Assume that $R(X)^{U}$ is a hypersurface, the elements of its minimal system of homogeneous generators have weights $\nu_{1}, \ldots, \nu_{d}$ and multidegrees $\left(\bar{m}_{1}, \bar{n}_{1}\right), \ldots,\left(\bar{m}_{d}, \bar{n}_{d}\right)$, and the determining relation has weight $\nu_{0}$ and multidegree $\left(\bar{m}_{0}, \bar{n}_{0}\right)$. Then the following decomposition holds:

$$
\begin{aligned}
& V(\lambda) \otimes V(\mu)
\end{aligned}
$$

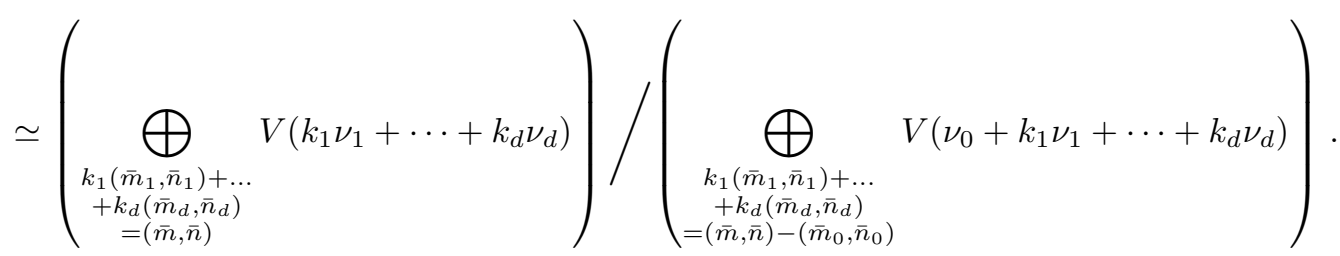

One can take an arbitrary embedding of the subrepresentation (by which we take the quotient) in the ambient representation, because the $G$-module structure of the quotient representation is independent of the embedding. Thus, the multiplicity of each irreducible representation $V(\nu)$ in $V(\lambda) \otimes V(\mu)$ is the difference of multiplicities of $V(\nu)$ in the ambient representation and the subrepresentation.

Proof. We denote a minimal system of homogeneous generators by $f_{1}, \ldots, f_{d}$ and the determining relation by $F\left(f_{1}, \ldots, f_{d}\right)=0$. Consider the exact sequence

$$
0 \rightarrow(F) \longrightarrow \mathbb{C}\left[t_{1}, \ldots, t_{d}\right] \stackrel{\varphi}{\longrightarrow} R(X)^{U} \rightarrow 0,
$$


where $\varphi\left(t_{i}\right)=f_{i}$. We equip the algebra $\mathbb{C}\left[t_{1}, \ldots, t_{d}\right]$ of polynomials with a $\mathbb{Z}^{r+t+l_{-}}$ grading, where $l=\operatorname{rk} G$, by assigning multidegree $\left(\bar{m}_{i}, \bar{n}_{i}\right)$ and weight $\nu_{i}$ to each variable $t_{i}$. Then

$$
\operatorname{dim} R(X)_{(\bar{m}, \bar{n}), \nu}^{U}=\operatorname{dim} \mathbb{C}\left[t_{1}, \ldots, t_{d}\right]_{(\bar{m}, \bar{n}), \nu}-\operatorname{dim}(F)_{(\bar{m}, \bar{n}), \nu} .
$$

The multiplicity of $V(\nu)$ in $V(\lambda) \otimes V(\mu)$ is $\operatorname{dim} R(X)_{(\bar{m}, \bar{n}), \nu}^{U}$, the multiplicity in the ambient representation is $\operatorname{dim} \mathbb{C}\left[t_{1}, \ldots, t_{d}\right]_{(\bar{m}, \bar{n}), \nu}$, and the multiplicity in the subrepresentation is $\operatorname{dim}(F)_{(\bar{m}, \bar{n}), \nu}$.

Now let us explain the above-introduced notions for the case of classical groups. Let $I=\left\{\alpha_{k}\right\}$. The cases of $G=\mathrm{SO}_{2 l+1}$ with $k=l$ and $G=\mathrm{SO}_{2 l}$ with $k=l-1, l$ will be considered later. In the other cases, $G / P_{I}^{-}$is the Grassmannian of $(n-k)$-dimensional subspaces for $G=\mathrm{SL}_{n}$ and the isotropic Grassmannian of $k$-dimensional subspaces for $G=\mathrm{Sp}_{n}, \mathrm{SO}_{n}$. The Plücker coordinate (the coefficient of the basis polyvector $e_{i_{1}} \wedge$ $\cdots \wedge e_{i_{m}}$ ) of the $m$-dimensional space in a flag belonging to $G / P^{-}$will be denoted by $r_{i_{1}, \ldots, i_{m}}$ (and by $s_{i_{1}, \ldots, i_{m}}$ for the points of $G / Q^{-}$). The sections of line bundles over $G / P_{I}^{-}$are homogeneous forms of the Plücker coordinates. The linear forms of the Plücker coordinates are sections of the bundle $\mathcal{O}\left(\Delta_{P, k}\right)$. The Schubert divisor $\Delta_{P, k}$ is the complement of the $B$-orbit of the point $e_{n} \wedge \cdots \wedge e_{k+1}$ for the case of $\mathrm{SL}_{n}$ and of the point $e_{n} \wedge \cdots \wedge e_{n-k+1}$ for the case of $G=\mathrm{Sp}_{n}, \mathrm{SO}_{n}$. The canonical section of the Schubert divisor is $r_{n, \ldots, k+1}$ for the case of $\mathrm{SL}_{n}$ and $r_{n, \ldots, n-k+1}$ for the case of $G=\mathrm{Sp}_{n}, \mathrm{SO}_{n}$. The weight of this section is equal to $\omega_{k}$.

Now consider the remaining cases. For $G=\mathrm{SO}_{2 l+1}$ with $k=l$, the variety $G / P_{I}^{-}$ is the isotropic Grassmannian of $l$-dimensional subspaces. For $G=\mathrm{SO}_{2 l}$ with $k=$ $l-1, l$, the flag variety $G / P_{I}^{-}$is the $G$-orbit of the points $\left\langle e_{n} \wedge \cdots \wedge e_{l+2} \wedge e_{l}\right\rangle$ and $\left\langle e_{n} \wedge \cdots \wedge e_{l+2} \wedge e_{l+1}\right\rangle$, respectively, in $\mathbb{P}\left(\wedge^{l} \mathbb{C}^{n}\right)$. The union of these orbits is the isotropic Grassmannian. In contrast to the cases considered above, it is not for all line bundles over $G / P_{I}^{-}$that the sections are homogeneous forms of the Plücker coordinates, because the Plücker coordinates are sections of the bundle $\mathcal{O}\left(2 \Delta_{P, k}\right)$. The squares of the sections corresponding to the Schubert divisors are equal to $r_{n, \ldots, l+2, l}$ for $k=l-1$ and $r_{n, \ldots, l+1}$ for $k=l$. The weights of the squared sections are $2 \omega_{k}$.

Note that for $G=\mathrm{SO}_{2 l}$ the Grassmannian of $(l-1)$-dimensional isotropic subspaces is not the flag variety corresponding to a maximal parabolic subgroup. Subspaces of dimension $l-1$ occur whenever $\alpha_{l-1}, \alpha_{l} \in I$. The Plücker coordinates of a subspace of dimension $l-1$ for $G=\mathrm{SO}_{2 l}$ are sections of the bundle $\mathcal{O}\left(\Delta_{P, l-1}+\Delta_{P, l}\right)$.

For $G=\mathrm{SO}_{n}, \mathrm{Sp}_{n}$, the Plücker coordinates of the subspaces $W$ and $W^{\perp}$ are sections of one and the same line bundle.

For the group $\mathrm{SO}_{n}$, the following notation will be convenient for us: $\pi_{i}:=\varepsilon_{1}+\cdots+\varepsilon_{i}$; $\pi_{l}^{\prime}:=\varepsilon_{1}+\cdots+\varepsilon_{l-1}-\varepsilon_{l}$. Then the weight of the Plücker coordinate $r_{n, \ldots, n-k+1}$ is $\pi_{k}$ for every $k$, and the weight of $r_{n, \ldots, l+2, l}$ is $\pi_{l}^{\prime}$. These weights can be expressed via the fundamental weights as follows: for $\mathrm{SO}_{2 l+1}$ one has $\pi_{i}=\omega_{i}, i<l$, and $\pi_{l}=2 \omega_{l}$; for $\mathrm{SO}_{2 l}$, one has $\pi_{i}=\omega_{i}, i<l-1, \pi_{l-1}=\omega_{l-1}+\omega_{l}, \pi_{l}=2 \omega_{l}$, and $\pi_{l}^{\prime}=2 \omega_{l-1}$.

\section{U-INVARIANTS OF COX RINGS}

Now let us describe the structure of algebras of $U$-invariants for the Cox rings of low-complexity varieties. Note that the group $\operatorname{Pic}(X)$ of a smooth complete rational $G$-variety $X$ is finitely generated and free [7, Section 17], and so the Cox ring $R(X)$ is well defined. 
Theorem 3. Let $X$ be a smooth complete variety of complexity 0 , and let $D_{1}, \ldots, D_{d}$ be all simple $B$-invariant divisors on $X$. Then the algebra $R(X)^{U}$ is freely generated by the canonical sections $s_{D_{i}}$.

Proof. Let us prove that the $s_{D_{i}}$ generate $R(X)^{U}$. Every element of $R(X)^{U}$ can be represented as a sum of sections of pairwise nonisomorphic bundles; these sections are $U$ invariant as well. Let us decompose a $U$-invariant section into a sum of $B$-semi-invariant sections. Hence it suffices to prove the assertion for an arbitrary $B$-semi-invariant section $s$ of a line bundle over $X$. Its divisor is a linear combination of the form $\operatorname{div} s=\sum a_{i} D_{i}$. Then $s$ is proportional to the product $\prod s_{D_{i}}^{a_{i}}$ over all $i=1, \ldots, d$.

It remains to prove that the $s_{D_{i}}$ are algebraically independent. First, note that $\mathbb{C}(X)^{B}=\mathbb{C}$. Indeed, each $f \in \mathbb{C}(X)^{B}$ is constant on the open orbit and hence on the entire $X$. If there exists a nontrivial relation between $s_{D_{i}}$, then there exist two nonproportional monomials in $s_{D_{i}}$ of the same weight and the same multidegree. The ratio of these monomials specifies a nonconstant $B$-invariant rational function on $X$, which contradicts the relation $\mathbb{C}(X)^{B}=\mathbb{C}$.

A similar theorem holds for varieties of complexity 1. Recall that we have introduced the notion of an exceptional point on $\mathbb{P}^{1}$ in Section 3. One definition is that a point $z=(p: q) \in \mathbb{P}^{1}$ is exceptional if $\operatorname{div}\left(q F-p F^{\prime}\right)$ is not simple.

Theorem 4. Let $X$ be a smooth complete rational variety of complexity 1 , let $D_{i}$ be all exceptional B-invariant divisors, and let $F$ and $F^{\prime}$ be sections as in Section 3. Then $R(X)^{U}$ is generated by the canonical sections $s_{D_{i}}, F$, and $F^{\prime}$. If there exist at least two exceptional points in $\mathbb{P}^{1}$, then one can remove $F$ and $F^{\prime}$ from the set of generators. The ideal of relations is generated by relations of the form

$$
q F-p F^{\prime}=\prod_{z_{D_{i}}=(p: q)} s_{D_{i}}^{h_{D_{i}}},
$$

where $(p: q)$ is an exceptional point of $\mathbb{P}^{1}$.

Proof. The fact that $R(X)^{U}$ is finitely generated can be shown by analogy with the preceding proof. Let $s$ be an arbitrary $B$-semi-invariant section. We represent the divisor of the section in the form of a linear combination of simple divisors,

$$
\operatorname{div} s=\sum a_{i} D_{i}+\sum a_{(p: q)} \operatorname{div}\left(q F-p F^{\prime}\right)
$$

then $s$ is proportional to

$$
\prod s_{D_{i}}^{a_{i}} \cdot \prod\left(q F-p F^{\prime}\right)^{a_{(p: q)}}
$$

If there exist at least two exceptional points, then $F$ and $F^{\prime}$ can be expressed via the $s_{D_{i}}$; this is easily seen from the relations described in the theorem.

Now let us find the relations in the algebra $R(X)^{U}$. We know that, for an appropriate normalization of the sections $s_{D_{i}}$, one has the relations

$$
q F-p F^{\prime}=\prod_{z_{D_{i}}=(p: q)} s_{D_{i}}^{h_{D_{i}}},
$$

where $(p: q)$ is an exceptional point. Let us prove that these relations generate all relations. Consider an arbitrary multidegree- and weight-homogeneous relation

$$
\prod s_{D_{i}}^{a_{i}} f\left(F, F^{\prime}\right)+\prod s_{D_{i}}^{b_{i}} g\left(F, F^{\prime}\right)+\cdots=0
$$

where $f, g, \ldots$ are homogeneous polynomials in $F$ and $F^{\prime}$. Somewhat later, we will show that, in view of the relations described above,

$$
\prod s_{D_{i}}^{a_{i}} f\left(F, F^{\prime}\right)+\prod s_{D_{i}}^{b_{i}} g\left(F, F^{\prime}\right)
$$


can be replaced by

$$
\prod s_{D_{i}}^{c_{i}} h\left(F, F^{\prime}\right)
$$

Thus, we can step by step reduce the number of terms of the form

$$
\prod s_{D_{i}}^{a_{i}} f\left(F, F^{\prime}\right)
$$

in the original relation. Eventually, we reduce the original relation to a relation of the form

$$
\prod s_{D_{i}}^{a_{i}} f\left(F, F^{\prime}\right)=0
$$

which only holds for $f=0$.

It remains to show that

$$
\prod s_{D_{i}}^{a_{i}} f\left(F, F^{\prime}\right)+\prod s_{D_{i}}^{b_{i}} g\left(F, F^{\prime}\right)
$$

can be replaced by

$$
\prod s_{D_{i}}^{c_{i}} h\left(F, F^{\prime}\right)
$$

Set

$$
s_{a}=\prod s_{D_{i}}^{a_{i}} f\left(F, F^{\prime}\right), \quad s_{b}=\prod s_{D_{i}}^{b_{i}} g\left(F, F^{\prime}\right) .
$$

Since $s_{a}$ and $s_{b}$ have the same weights and multidegrees, it follows that their ratio is some function in $\mathbb{C}(X)^{B} \simeq \mathbb{C}\left(\mathbb{P}^{1}\right)$; the homogeneous coordinates on $\mathbb{P}^{1}$ are $F$ and $F^{\prime}$. Then $\operatorname{div} s_{a}$ and $\operatorname{div} s_{b} \operatorname{differ}$ by the divisor of a rational function of $F$ and $F^{\prime}$, and one has the relation

$$
\sum_{i} a_{i} D_{i}+\operatorname{div} g_{a}\left(F, F^{\prime}\right)=\sum_{i} b_{i} D_{i}+\operatorname{div} g_{b}\left(F, F^{\prime}\right)
$$

where $g_{a}$ and $g_{b}$ are homogeneous polynomials. Consider the multiplicities of the exceptional divisors in this relation. If $D_{i}$ is an exceptional divisor with $h_{D_{i}}=0$, then $a_{i}=b_{i}$. Let $z$ be an exceptional point of $\mathbb{P}^{1}$. In the relation, let us single out the divisors $D$ with $z_{D}=z\left(\right.$ and $\left.h_{D}>0\right)$. By rearranging the terms, we obtain

$$
\sum_{\substack{z_{D_{i}}=z \\ h_{D_{i}}>0}}\left(a_{i}-b_{i}\right) D_{i}=\operatorname{div}\left(q F-p F^{\prime}\right)^{k}=k \sum_{\substack{z_{D_{i}}=z \\ h_{D_{i}}>0}} h_{D_{i}} D_{i},
$$

where $(p: q)=z$ and $k \in \mathbb{Z}$.

We can assume that $k \geq 0$. Then $a_{i} \geq b_{i}$ for all $i$ such that $z_{D_{i}}=z$ and $h_{D_{i}}>0$. In $s_{a}$, let us replace the product

$$
\prod_{z_{D_{i}}=z} s_{D_{i}}^{a_{i}} \quad \text { by } \prod_{z_{D_{i}}=z} s_{D_{i}}^{b_{i}} \cdot\left(q F-p F^{\prime}\right)^{k} .
$$

Then we see that the degrees of the sections $s_{D_{i}}$ in $s_{a}$ and $s_{b}$ coincide for $i$ such that $z_{D_{i}}=z$ and $h_{D_{i}}>0$. By carrying out this replacement for all exceptional points of $\mathbb{P}^{1}$, we see that from $s_{a}$ and $s_{b}$ one can extract a factor of the form $\prod s_{D_{i}}^{c_{i}}$ such that the remaining factors are polynomials in $F$ and $F^{\prime}$.

The following theorem holds for double flag varieties of complexity 1 .

Theorem 5. Let the complexity of a double flag variety $X$ of a classical group $G$ be equal to 1 . Then the algebra $R(X)^{U}$ is either free or is a hypersurface.

Proof. This result follows from the explicit description (Sections 7-9) of $B$-invariant divisors for each of these varieties. We find that there exist two or three exceptional points in each of these cases. By the preceding theorem, $R(X)^{U}$ is free if there are two exceptional points and is a hypersurface if there are three exceptional points. 
Remark 1. A special case of this theorem follows from a result earlier obtained by Panyushev [4, 6.5]. He proved that $R(X)^{U}$ is a hypersurface for the case of maximal parabolic subgroups.

\section{Method For COMputing invariants of the Cox Rings FOR THE CLASSICAL GROUPS}

In the preceding section, we have shown that to find generators and relations in $R(X)^{U}$ it suffices to know the structure of simple $B$-invariant divisors. In Section 6.1, we present a method for finding simple $B$-invariant divisors. We specify $B$-invariant divisors by geometric conditions. In Section 6.2, we explain how these conditions should be understood and how, starting from the geometric setting, one can find the canonical section corresponding to the divisor or, in other words, find the equation of the divisor in the Plücker coordinates. In Section 6.3, we show how to verify that a section constructed according to a geometric form specifies a simple divisor or its multiplicity. Section 6.4 gives a more detailed discussion of the structure of simple divisors on double flag varieties of complexity 1 . We show how to determine which of the simple divisors constructed by the method in Section 6.1 are exceptional and which are parametric and explain how to specify parametric divisors. Section 6.5 clarifies the form in which we will present the result for each of the cases (in Sections 7-9).

6.1. Method for finding $B$-invariant divisors. The main idea underlying the search for $B$-invariant divisors is to try to reduce an arbitrary point in $X=G / P^{-} \times G / Q^{-}$by the action of $B$ to a canonical form and find conditions under which this is impossible. It is these conditions that specify $B$-invariant divisors in the complement of the open $B$-orbit in the case of complexity 0 and $B$-invariant divisors in the complement of some parametric family of $B$-orbits in the case of complexity 1.

Recall that if the subgroup $P$ is not exceptional, then the subspaces in a flag in $G / P^{-}$are denoted by $R_{i}$, where the subscript stands for dimension. If the subgroup is exceptional (which is possible in the case of $G=\mathrm{SO}_{2 l}$ ), then the subspace of dimension $l$ is denoted by $R_{l}^{\prime}$, while the notation for the subspaces of other dimensions is preserved. For the double flag varieties $X=G / P^{-} \times G / Q^{-}$, the subspaces in the flags corresponding to points in $G / Q^{-}$are denoted in a similar way by $S_{j}$ or $S_{l}^{\prime}$. We assume that the point does not lie in Schubert divisors. Then the point can be reduced to a form such that $R_{i}=\left\langle e_{n}, \ldots, e_{n-i+1}\right\rangle$ for all $R_{i}$ in the flag and $R_{l}^{\prime}=\left\langle e_{n}, \ldots, e_{l+2}, e_{l}\right\rangle$ (if the subspace $R_{l}^{\prime}$ occurs in the flag) or to a form such that $S_{j}=\left\langle e_{n}, \ldots, e_{n-j+1}\right\rangle$ for all $S_{j}$ in the flag and $S_{l}^{\prime}=\left\langle e_{n}, \ldots, e_{l+2}, e_{l}\right\rangle$ (if this subspace occurs in the flag). We reduce the point to one of the two forms; to be definite, assume that this is the first form. We carry out the subsequent transformations in such a way that the subspaces $R_{i}$ (and $R_{l}^{\prime}$ ) do not change any more.

If the flag does not contain the subspace $S_{l}^{\prime}$, then we choose the bases in the subspaces $S_{j}$ as shown in Figure 6.1. In this figure, the columns of the matrices depict the basis vectors in the subspaces, and the vertical lines bound the subspaces in the flag on the right. If the subspace $S_{l}^{\prime}$ occurs in the flag, then we reduce the point to the form obtained from Figure 6.1 (with an $l$-dimensional subspace present in the flag) by the transposition of two middle rows. The coordinates of the basis vectors in the positions shown by the asterisks are uniquely determined.

Next, we use the action of the group $B$ to zero the coordinates shown by the asterisks. Having zeroed some of the coordinates, we can encounter a situation in which we cannot always zero one of the coordinates. The resulting points will be referred to as points of form 1 . The coordinate that cannot always be zeroed will be denoted by $x_{1}$. Note that the position of this coordinate is not uniquely determined; it depends on the choice of 


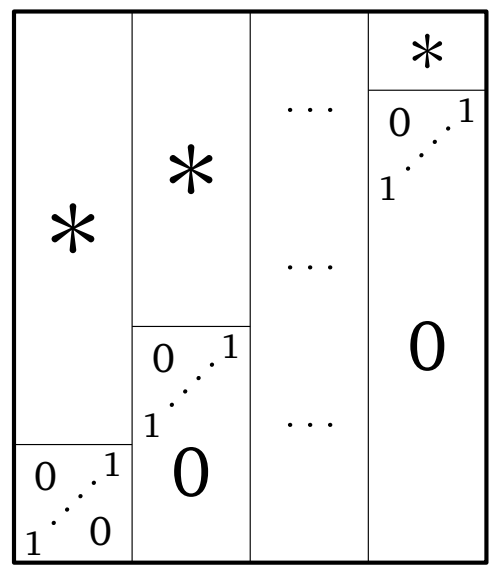

FiguRE 6.1

the sequence of coordinates to be zeroed consecutively. The equation $x_{1}=0$ determines a $B$-invariant divisor $D_{1}$ in the following sense: the $B$-invariant divisor is defined as the closure of the union of $B$-orbits of form 1 points such that $x_{1}=0$. We define this divisor by a $B$-invariant geometric condition (on the open set obtained by deleting the Schubert divisors); see Section 6.2.

In what follows, we assume that the point does not lie in the divisor $D_{1}$. For such points, one has $x_{1} \neq 0$. If the complexity is 0 , the one can ensure that $x_{1}=1$. If the complexity is 1 , then this is not always the case; we zero the coordinates in an order such that the coordinate that cannot be made equal to 1 has the maximum possible number. We can further zero some coordinates (in such a way that the point still has form 1). We again find a coordinate that we cannot always zero and denote it by $x_{2}$. The resulting points will be called points of form 2 . Note that every point of form 2 is also a point of form 1 by definition. The equation $x_{2}=0$ again determines a divisor, $D_{2}$, which is the closure of the union of orbits of form 2 points such that $x_{2}=0$.

The subsequent procedure is completely similar: we specify the divisor by a $B$ invariant geometric condition on the open set obtained by deleting the Schubert divisors and the divisor $D_{1}$, delete the points of the divisor $D_{2}$, ensure that $x_{2}=1$ on the resulting set (if this is possible), zero the coordinates that we can zero, etc. It will sometimes be convenient for us to denote the "nonzeroable" coordinates by $x_{j}^{\prime}$ and $x_{j}^{\prime \prime}$ rather than $x_{i}$ (and the corresponding divisors by $D_{j}^{\prime}$ and $D_{j}^{\prime \prime}$ rather than $D_{i}$ ).

If the complexity of $X$ is 0 , then this method gives all simple $B$-invariant divisors. Indeed, we have deleted some divisors and reduced the point outside them to a single canonical form. In other words, the complement of these divisors is an open $B$-orbit, and hence there exist no other $B$-invariant divisors.

If the complexity of $X$ is 1 , then the divisors that we have found do not necessarily exhaust all exceptional divisors, and some of them cam be parametric. Nevertheless, we have found divisors such that the $B$-orbits in their complement are parametrized by the numbers $z:=x_{m} \in \mathbb{C} \backslash\{0\} \simeq \mathbb{P}^{1} \backslash\{0, \infty\}$, where $x_{m}$ is the coordinate that we cannot always make equal to unity. It is the closures of these orbits that are the remaining simple $B$-invariant divisors.

This algorithm is illustrated in detail for case 1 in Section 7.1.

We postpone the method for a more detailed study of divisors in the case of complexity 1 until Section 6.4. 
6.2. On the geometric form of notation. We will represent the conditions specifying the divisors in geometric form, i.e., in terms of the arrangement of subspaces. This notation is convenient: first, it is more concise and visual, and second, this setting readily implies $B$-invariance.

Let us describe what the geometric conditions look like. Assume momentarily that $G=\mathrm{SL}_{n}, \mathrm{Sp}_{n}$, or $\mathrm{SO}_{2 l+1}$. Let $E_{k}=\left\langle e_{1}, \ldots, e_{k}\right\rangle$ be a $B$-invariant subspace. In what follows, we define admissible expressions recursively. The subspaces $R_{p}$ and $S_{q}$ play the role of variables and are elementary admissible expressions. If $W$ and $W^{\prime}$ are admissible expressions, then so are $W+W^{\prime}$ and $W \cap E_{k}$. The substitution of specific subspaces $R_{p}$ and $S_{q}$ into admissible expressions gives some subspaces in $\mathbb{C}^{n}$. A geometric condition for a divisor has one of the following forms: $W \neq 0$ or $W \perp W^{\prime}$, where $W$ and $W^{\prime}$ are admissible expressions.

For $G=\mathrm{SO}_{2 l}$, we use $E_{k}$ as well as the $B$-invariant subspace $E_{l}^{\prime}=\left\langle e_{1}, \ldots, e_{l-1}, e_{l+1}\right\rangle$ in the geometric conditions. The subspaces $R_{l}^{\prime}$ (and $S_{l}^{\prime}$ ) occur for the exceptional parabolic subgroups. The geometric specification of a flag that contains the subspaces $R_{l-1}$ and $R_{l}$ will use some subspace also denoted by $R_{l}^{\prime}$ and defined as the unique isotropic subspace of dimension $l$ other than $R_{l}$ for which $R_{l-1} \subset R_{l}^{\prime} \subset R_{l-1}^{\perp}=R_{l+1}$. (Similar notation is used for flags in $G / Q^{-}$.)

Now let us explain how to obtain an equation in the Plücker coordinates from the geometric notation. To the admissible expressions occurring in the geometric notation, we assign some polyvectors depending on the Plücker coordinates of $R_{p}$ and $S_{q}$ by the following rule. Momentarily, let $G=\mathrm{SL}_{n}, \mathrm{Sp}_{n}$, or $\mathrm{SO}_{2 l+1}$. To the subspaces $R_{p}$ and $S_{q}$, we assign the polyvectors $\sum r_{i_{1}, \ldots, i_{p}} e_{i_{1}} \wedge \cdots \wedge e_{i_{p}}$ and $\sum s_{j_{1}, \ldots, j_{q}} e_{j_{1}} \wedge \cdots \wedge e_{j_{q}}$ of degree $p$ and $q$, respectively, determining these subspaces. Let a polyvector $\sum w_{i_{1}, \ldots, i_{r}} e_{i_{1}} \wedge \cdots \wedge e_{i_{r}}$ correspond to an admissible expression $W$, and let $E_{m}$ be a minimal $B$-invariant subspace (of the $E_{k}, k \leq n$ ) such that $W \subseteq E_{m}$ for any values of the variables $R_{p}$ and $S_{q}$ substituted into the expression $W$. Then to the expression $W \cap E_{k}$ (we write out the geometric conditions in such a way that $k<m$ ) we assign the polyvector $\sum w_{m, m-1, \ldots, k+1, j_{1}, \ldots, j_{r-m+k}} e_{j_{1}} \wedge \cdots \wedge e_{j_{r-m+k}}$, and to the expression $W+W^{\prime}$ we assign the exterior product of the polyvectors corresponding to $W$ and $W^{\prime}$. If the subspaces $R_{p}$ and $S_{q}$ are in a sufficiently general position, then these polyvectors correspond to the intersection and sum of subspaces in the sense of Plücker embeddings of the corresponding Grassmannians. Let the geometric condition have the form $W \neq 0$. The conditions are chosen in such a way that the polyvector corresponding to $W$ has degree 0; i.e., it is a number depending on the Plücker coordinates. By equating this number with 0 , we obtain the equation of the divisor in the Plücker coordinates. Now consider the other form of the condition, $W \perp W^{\prime}$. The geometric conditions are chosen in such a way that some polyvectors of degree 1 , i.e., vectors in $\mathbb{C}^{n}$, correspond to the expressions $W$ and $W^{\prime}$. By writing out the orthogonality condition for these vectors, we obtain the equation of the divisor.

Now let $G=\mathrm{SO}_{2 l}$. The algorithm is similar to that in the case of $G=\mathrm{SL}_{n}, \mathrm{Sp}_{n}, \mathrm{SO}_{2 l+1}$ but has a few additions. If $E_{m}$ is a minimal $B$-invariant subspace (now of $E_{k}, k \leq n$, and $E_{l}^{\prime}$ ) such that $W \subseteq E_{m}$, then we assign a polyvector to the expression $W \cap E_{k}$ just as before, and to the expression $W \cap E_{l}^{\prime}$ we assign $\sum w_{m, m-1, \ldots, l+2, l, j_{1}, \ldots, j_{r-m+l}} e_{j_{1}} \wedge \cdots \wedge$ $e_{j_{r-m+l}}$. If $E_{l}^{\prime}$ is a minimal $B$-invariant subspace (of $E_{k}, k \leq n$, and $E_{l}^{\prime}$ ) such that $W \subseteq E_{l}^{\prime}$, then to the expression $W \cap E_{k}$ we assign the polyvector $\sum w_{l+1, l-1, l-2, \ldots, k+1, j_{1}, \ldots, j_{r-l+k}} e_{j_{1}}$ $\wedge \cdots \wedge e_{j_{r-l+k}}$.

The method for constructing the equation from the geometric condition is also analyzed in case 1 in Section 7.1. 
Now let us explain how to find the multidegree of the corresponding equation from the geometric condition. To find the degree in the Plücker coordinates of the subspace $R_{p}$, one should count the number of occurrences of $R_{p}$ (and of $R_{p}^{\perp}$ in the orthogonal and symplectic cases) in the geometric condition. The degree in the Plücker coordinates of $S_{q}$ can be determined in a similar way. The multidegree in the Plücker coordinates will be denoted by $(\ldots)_{P l}$. Let us describe the relationship between the multidegree in the Plücker coordinates and the multidegree in the Picard group. Let $m_{i}$ be the degree in $\Delta_{P, i}$ in $\operatorname{Pic}(X)$, let $p_{i}$ be the degree in the Plücker coordinates of the space $R_{i}$, and let $p_{l}^{\prime}$ be the degree in the Plücker coordinates of the space $R_{l}^{\prime}$. Then $m_{i}=p_{n-i}$ for the group $\mathrm{SL}_{n}, m_{i}=p_{i}$ for the group $\mathrm{Sp}_{n}$, and the following formulas hold for the group $\mathrm{SO}_{2 l+1}$ : $m_{i}=p_{i}, i<l$, and $m_{l}=2 p_{l}$. For $\mathrm{SO}_{2 l}$, one has $m_{i}=p_{i}, i \leq l-2, m_{l-1}=p_{l-1}+2 p_{l}^{\prime}$, and $m_{l}=p_{l-1}+2 p_{l}$.

6.3. Methods for verifying the simplicity of divisors. We have described at the beginning of this section how to seek simple $B$-invariant divisors. We have sought a geometric condition on $D_{i}$ such that the set of zeros of the corresponding form $f_{i}$ in the Plücker coordinates coincides with $D_{i}$ on

$$
U_{i}:=X \backslash\left(\Delta_{P, i_{1}} \cup \cdots \cup \Delta_{P, i_{r}} \cup \Delta_{Q, j_{1}} \cup \cdots \cup \Delta_{Q, j_{t}} \cup D_{1} \cup \cdots \cup D_{i-1}\right) ;
$$

i.e., $\operatorname{div} f_{i}$ restricted to $U_{i}$ is the multiplicity of $D_{i}$. We can find the weight and multidegree of $f_{i}$, but to find the weight and multidegree of $s_{D_{i}}$ correctly, one has to know $\operatorname{div} f_{i}$ on the entire $X$. We proceed as follows: first, we make sure that $\operatorname{div} f_{i}$ does not contain the Schubert divisors and $D_{1}, \ldots, D_{i-1}$, and then we find the multiplicity of $D_{i}$ in $\operatorname{div} f_{i}$.

Let us explain how to verify that $\operatorname{div} f_{i}$ does not contain the Schubert divisors and $D_{1}, \ldots, D_{i-1}$. First, we use a "weight-and-degree" argument: if $\operatorname{div} f_{i}$ contains $D$, then the multidegree of $f_{i}$ is greater than or equal to the multidegree of $s_{D}$ (componentwise), and the difference between the weights of $f_{i}$ and $s_{D}$ is a dominant weight. Second, we can exhaust cases in an order such that the $B$-invariant divisor on "smaller" double flag varieties will already be known. (The "smaller" varieties for $X=G / P_{I}^{-} \times G / P_{J}^{-}$ are varieties $X^{\prime}=G / P_{I^{\prime}}^{-} \times G / P_{J^{\prime}}^{-}$different from $X$ such that $I^{\prime} \subseteq I$ and $J^{\prime} \subseteq J$; i.e., there exists a $G$-equivariant surjective morphism $X \rightarrow X^{\prime}$.) Thus, we know the weights and multidegrees of the canonical sections corresponding to $B$-invariant divisors whose multidegree has at least one zero component.

Using these two ideas, we obtain the following algorithm for verifying that $\operatorname{div} f_{i}$ does not contain $D$, where $D$ is a Schubert divisor or one of the $D_{j}, j<i$. Let $\nu$ be the difference of weights of $f_{i}$ and $s_{D}$, and let $(\bar{m}, \bar{n})$ be the difference of their multidegrees. If there is a negative component in $(\bar{m}, \bar{n})$ or $\nu$ is not a dominant weight, then the verification is terminated. Now assume that there are no negative components in $(\bar{m}, \bar{n})$ and $\nu$ is a dominant weight. Further, assume that there is a zero component in $(\bar{m}, \bar{n})$. Then $\operatorname{div} f_{i}-D$ is the preimage of a divisor on a "smaller" double flag variety. One should verify that there are no divisors with this multidegree and weight of the canonical section on the corresponding smaller variety. This argument suffices in a majority of cases. This method is in particular used in case 1 in Section 7.1.

For some divisors $D_{i}$, one can prove that the multidegrees of the corresponding canonical sections do not have zero components. The proof is based on the knowledge of the divisors on smaller flag varieties. Thus, if we have already found all simple divisors such that the multidegrees of their sections have zero components, then the sections corresponding to the remaining divisors do not have zero components. Then the verification in the preceding algorithm can be terminated once $(\bar{m}, \bar{n})$ has zero components. This method is used, e.g., in case 5(a) in Section 8.2. 
If this argument does not suffice, then we find a point $x \in D_{j}$ such that $f_{i}(x) \neq 0$. This method is used, e.g., in case 5(b) in Section 8.2.

Now let us explain how to find the multiplicity of $D_{i}$ in $\operatorname{div} f_{i}$. Assume that we have already verified that the Schubert divisors and the $D_{j}, j<i$, do not occur in $\operatorname{div} f_{i}$. Here we can also use a "weight-and-degree" argument. If $\operatorname{div} f_{i}=c D_{i}$, then the components of the multidegree should be multiples of $c$, and the weight should be the $c$-multiple of a dominant weight. In a majority of cases, this method permits one to find that $c=1$.

Assume that the method has failed to find $c$. Let us restrict everything to the set $U_{i}$. Find a subgroup $H \subseteq B$ and a section $Z$ that transversally intersects each $H$-orbit at a single point and is invariant under some subgroup $H^{\prime} \subseteq B$ such that $B=H H^{\prime}$. Then $U_{i} \simeq H \times Z$, and $B$-invariant divisors on $U_{i}$ have the form $H \times D$, where $D$ is an $H^{\prime}$ invariant divisor on $Z$. Find a section such that the form $f_{i}$ has the simplest expression in the local coordinates on this section. Then we can find $c$ by analyzing the restriction $\left.f_{i}\right|_{Z}$. This method is illustrated by case 4 in Section 8.2 and case 1 in Section 9.1.

6.4. Determining the structure of divisors in the case of complexity 1 . Let $D_{1}, \ldots, D_{m}$ be all simple $B$-invariant divisors except for the Schubert divisors on a double flag variety of complexity 1 found by the algorithm in Section 6.1. Let us find two coprime monomials of one and the same weight and multidegree in the canonical sections of the Schubert divisor and $D_{1}, \ldots, D_{m}$ such that the parameter $z$ (viewed as a $B$-invariant rational function on $X$ ) is the ratio of these monomials. The existence of such monomials can be shown as follows. Since the $B$-orbits in the complement of the Schubert divisors and $D_{1}, \ldots, D_{m}$ are parametrized by numbers $z \in \mathbb{C} \backslash\{0\}$, it follows that the set consisting of the Schubert divisors and $D_{1}, \ldots, D_{m}$ contains all simple $B$ invariant divisors $D$ such that $z_{D}=0$ or $z_{D}=\infty$. Then we can take these monomials for $F$ and $F^{\prime}$.

Now we can determine which of the divisors $D_{1}, \ldots, D_{m}$ are parametric; these are exactly the divisors whose weight and multidegree coincide with $F$ and $F^{\prime}$. The divisor of the section $F-z F^{\prime}$ restricted to

$$
U_{m+1}:=X \backslash\left(\Delta_{P, i_{1}} \cup \cdots \cup \Delta_{P, i_{r}} \cup \Delta_{Q, j_{1}} \cup \cdots \cup \Delta_{Q, j_{t}} \cup D_{1} \cup \cdots \cup D_{m}\right)
$$

is the closure of the orbit corresponding to the parameter value $z$. The closure of such an orbit is an exceptional divisor if $\operatorname{div}\left(F-z F^{\prime}\right)$ considered on the entire $X$ contains one of the Schubert divisors or one of $D_{1}, \ldots, D_{m}$ or if $F-z F^{\prime}$ has order $\geq 2$ along this orbit. Otherwise, the closure of the orbit is a parametric divisor.

Instead of verifying this directly, we either show that all the closures of orbits in $U_{m+1}$ are parametric divisors or find an additional simple $B$-invariant divisor $D_{m+1}$ (which naturally coincides with the closure of one of the orbits of the parametric family) such that all closures of $B$-orbits in $U_{m+1} \backslash D_{m+1}$ are parametric divisors.

Let $\mathcal{D}_{1}$ be the set of those divisors in the set of Schubert divisors and $D_{1}, \ldots, D_{m}$ which do not occur in $\operatorname{div} F$ and $\operatorname{div} F^{\prime}$. For $D \in \mathcal{D}_{1}$, we wish to find out whether there exists a $z$ such that $\operatorname{div}\left(F-z F^{\prime}\right)$ contains $D$. By applying the "weight-and-degree" argument, we find that the desired $z$ fails to exist for some $D \in \mathcal{D}_{1}$.

Let $\mathcal{D}_{2} \subseteq \mathcal{D}_{1}$ be the set of remaining divisors. If $\mathcal{D}_{2}=\varnothing$, then all closures of orbits in $U_{m+1}$ are simple divisors that are either parametric or some multiple of the corresponding section is proportional to $F-z F^{\prime}$ for some $z$. Let $\mathcal{D}_{2} \neq \varnothing$. Let us find a new $B$ invariant divisor $D_{m+1}$ such that some monomial of the form $s_{D_{m+1}}^{a_{D_{m+1}}} \prod_{D \in \mathcal{D}_{2}} s_{D}^{a_{D}}$, where $a_{D_{m+1}}, a_{D}>0$, has the same multidegree and weight as $F$ and $F^{\prime}$. There is no general explanation why this is possible, but there exists a divisor with these properties in each of the cases considered in what follows. This monomial will be proportional to $F-z_{0} F^{\prime}$ for some $z_{0}$, and we have $z_{D}=z_{0}$ and $h_{D}=a_{D}>0$ for the divisors $D \in \mathcal{D}_{2} \cup\left\{D_{m+1}\right\}$. 
The simple divisors that are closures of orbits in $U_{m+1} \backslash D_{m+1}$ are either parametric or some multiple of the corresponding section is proportional to $F-z F^{\prime}$ for some $z$.

It remains to verify that $F-z F^{\prime}$ cannot have order $\geq 2$ along the closure of one of the orbits in $U_{m+1} \backslash D_{m+1}$. This again follows from the "weight-and-degree" argument: in the cases considered here (in Sections 7-9), the multidegree of $F$ and $F^{\prime}$ is not a multiple of another multidegree. This method is illustrated in case 6 in Section 7.2.

6.5. Form of representation of the result. The figures show a point in $G / P^{-} \times$ $G / Q^{-}$. The left figures correspond to the projection onto $G / P^{-}$, and the right figures correspond to the projection onto $G / Q^{-}$. The columns denote the basis vectors of the flag subspaces. Bold lines divide the subspaces; thus, the columns before the first bold line form a basis in the flag subspace of the least dimension, the columns before the second bold line form a basis in the flag subspace of the second least dimension, etc. The variables $x_{i}$ are arranged according to the algorithm given in Section 6.1. For the case of complexity 0 , the replacement of all $x_{i}$ in a figure by 1 gives a point of the canonical form. For the case of complexity 1, the replacement of all but the last $x_{i}$ in a figure by 1 and the last $x_{i}$ by $z$ gives a point of the canonical form in the $B$-orbit corresponding to the parameter $z$.

In each case, information about divisors is given in a table. For convenience, by $D_{P, i}$ and $D_{P, l}^{\prime}$ we denote the Schubert divisors of the flag variety whose points are the subspaces $R_{i}$ and $R_{l}^{\prime}$. Thus, $\Delta_{P, i}=D_{P, n-i}$ for the group $\mathrm{SL}_{n}$ and $\Delta_{P, i}=D_{P, i}$ for the groups $\mathrm{Sp}_{n}$ and $\mathrm{SO}_{2 l+1}$. For $\mathrm{SO}_{2 l}$, we have $\Delta_{P, i}=D_{P, i}, i \neq l-1$, and $\Delta_{P, l-1}=D_{P, l}^{\prime}$. For brevity, the sections $s_{D_{i}}$ will be denoted by $F_{i}$, and the sections $s_{D_{P, i}}$ and $s_{D_{P, l}^{\prime}}$ will be denoted by $F_{P, i}$ and $F_{P, l}^{\prime}$, respectively. The symbols $D_{Q, i}, D_{Q, l}^{\prime}, F_{Q, i}$, and $F_{Q, l}^{\prime}$ are defined in a similar way. For the cases of complexity 0 , the table includes the geometric conditions on divisors as well as the weights and multidegrees of the corresponding canonical sections for all simple $B$-invariant divisors except for the Schubert divisors, for which this information is already known from Section 4. For the cases of complexity 1, the table includes information about all exceptional divisors but the Schubert divisors and also the products of sections corresponding to exceptional divisors that have the same multidegree and weight as $F$ and $F^{\prime}$. Recall that if there are two products, then there exist two exceptional points and $R(X)^{U}$ is free. If there are three products, then there exist three exceptional points; these products satisfy a linear relation, and $R(X)^{U}$ is a hypersurface.

Note that, in the case of $\mathrm{SO}_{n}$, the geometric condition interpreted as an equation in Plücker coordinates sometimes specifies the squared canonical section. If the geometric condition does not specify a simple divisor, then we first write out the multidegree in the Plücker coordinates and the weight of the section corresponding to the geometric condition and then indicate the multidegree in the Picard group and the weight of the section corresponding to the simple divisor. Since the geometric condition additionally uses the subspaces $R_{l}^{\prime}$ and $S_{l}^{\prime}$, we see that the multidegree in the Plücker coordinates has an additional component in this case. The degree in the Plücker coordinates of these additional subspaces will be written after the degree in the Plücker coordinates of the subspaces $R_{l-1}$ and $R_{l}$ (or $S_{l-1}$ and $S_{l}$ ).

\section{THE GROUP $\mathrm{SL}_{n}$}

\subsection{Complexity 0.}

1. $\left(p_{1}, p_{2}\right),\left(q_{1}, q_{2}\right)$. Let $p=p_{1}$ and $q=q_{1}$. Without loss of generality, we can assume that $p \leq q$. 

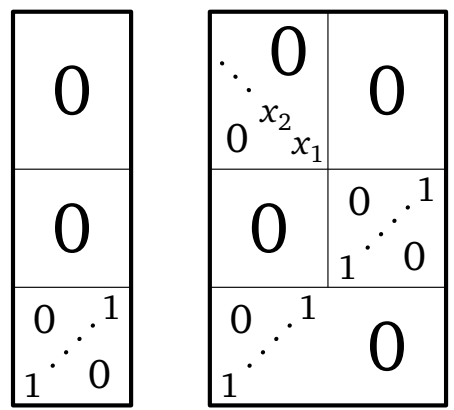

FiguRE 7.1.1

\begin{tabular}{|c|c|c|r|}
\hline & deg & Weight & Geometric condition \\
\hline$F_{k}$ & $(1,1)$ & $\omega_{p-k}^{*}+\omega_{q+k}^{*}$ & $\left\langle R_{p} \cap E_{n-p+k}+S_{q} \cap E_{n-p+k}\right\rangle \cap E_{n-q-k} \neq 0$, \\
& & & $k=1, \ldots, \min (p, n-q)$ \\
\hline
\end{tabular}

Let us explain why these geometric conditions determine all simple $B$-invariant divisors except for the Schubert divisors. Consider an arbitrary point $\left(R_{p}, S_{q}\right)$ in $G / P^{-} \times G / Q^{-}$. We will try to reduce it by the action of the group $B$ to the canonical form of points in the open orbit. We are interested in conditions under which this is impossible, i.e., conditions under which the point does not lie in the open orbit.

If $r_{n, \ldots, n-p+1} \neq 0$, then there exists a basis in $R_{p}$ such that the space $R_{p}$ has the form as shown in Figure 7.1.2a i.e., the columns of the matrix shown in the figure are the basis vectors in $R_{p}$. The condition $r_{n, \ldots, n-p+1}=0$ specifies the Schubert divisor; geometrically, this condition means that $R_{p} \cap E_{n-p} \neq 0$. In what follows, we assume that $r_{n, \ldots, n-p+1} \neq 0$. We can use the action of the Borel subgroup to reduce $R_{p}$ to the form shown in Figure 7.1.2b. We will ensure that $R_{p}$ does not change further under the subsequent transformations.

If $s_{n, \ldots, n-q+1} \neq 0$, i.e., if $S_{q}$ lies outside the corresponding Schubert divisor, then we choose a basis in $S_{q}$ such that $S_{q}$ has the form shown in Figure 7.1.2c. The action of $B$ can be used to reduce $S_{q}$ to the form shown in Figure 7.1.2d, If $x_{1} \neq 0$ (see Figure 7.1.2d), then $S_{q}$ can be reduced to the form shown in Figure 7.1.2e. Next, we look at the element $x_{2}$ (Figure 7.1.2e), etc. If all such elements are nonzero, then $S_{q}$ can be reduced to a canonical form (Figure 7.1.2f). Now let us find conditions under which the elements $x_{1}, x_{2}, \ldots$ are zero.

If $x_{k}=0$, then the determinant formed by the subcolumns highlighted in Figure $7.1 .2 \mathrm{~g}$ is zero. The subspaces spanned by the columns obtained from the highlighted ones by extension up and down coincide with $R_{p} \cap E_{n-p+k}$ and $S_{q} \cap E_{n-p+k}$, respectively. Since the determinant is zero, it follows that so is the Plücker coordinate multiplying $e_{n-p+k} \wedge \cdots \wedge e_{n-q-k+1}$ in the sum of these two subspaces, which implies that the intersection is nontrivial:

$$
\left\langle R_{p} \cap E_{n-p+k}+S_{q} \cap E_{n-p+k}\right\rangle \cap E_{n-q-k} \neq 0 .
$$

The polyvectors corresponding to the subspaces $R_{p}$ and $S_{q}$ are $\sum r_{i_{1}, \ldots, i_{p}} e_{i_{1}} \wedge \cdots \wedge e_{i_{p}}$ and $\sum s_{j_{1}, \ldots, j_{q}} e_{j_{1}} \wedge \cdots \wedge e_{j_{q}}$, respectively. Then the polyvector corresponding to the subspace $R_{p} \cap E_{n-p+k}$ is $\sum r_{n, \ldots, n-p+k+1, i_{p-k+1}, \ldots, i_{p}} e_{i_{p-k+1}} \wedge \cdots \wedge e_{i_{p}}$, and the polyvector corresponding to the subspace $S_{q} \cap E_{n-p+k}$ is $\sum s_{n, \ldots, n-p+k+1, j_{p-k+1}, \ldots, j_{q}} e_{j_{p-k+1}} \wedge \cdots \wedge e_{j_{q}}$; 


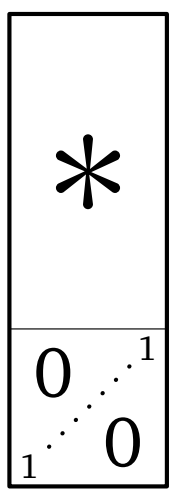

(a)

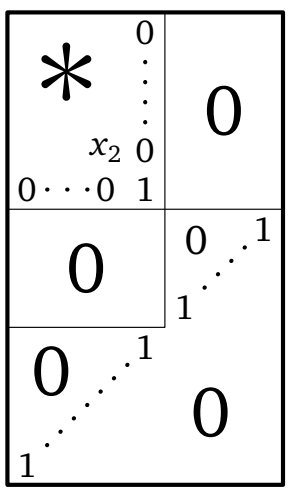

(e)

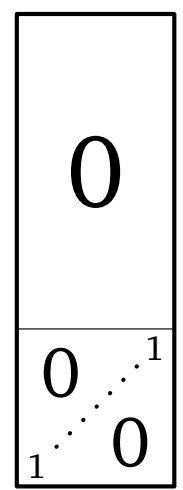

(b)

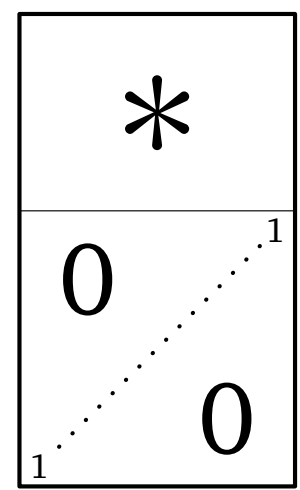

(c)

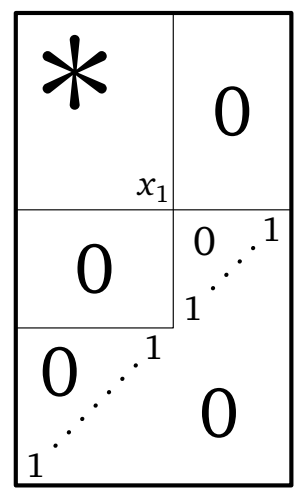

(d)

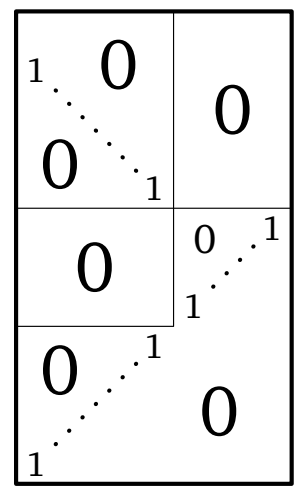

(f)
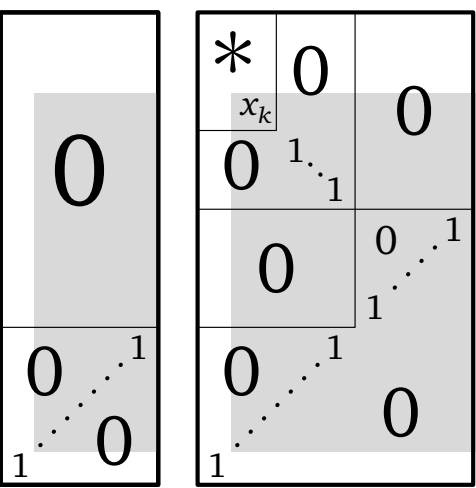

$(\mathrm{g})$

FiguRE 7.1.2.

the polyvector corresponding to the sum of these subspaces is

$\sum r_{n, \ldots, n-p+k+1, i_{p-k+1}, \ldots, i_{p}} s_{n, \ldots, n-p+k+1, j_{p-k+1}, \ldots, j_{q}} e_{i_{p-k+1}} \wedge \cdots \wedge e_{i_{p}} \wedge e_{j_{p-k+1}} \wedge \cdots \wedge e_{j_{q}}$.

By equating the Plücker coordinate multiplying $e_{n-q-k+1} \wedge \cdots \wedge e_{n-p+k}$ with zero, we obtain the following equation for the divisor:

$$
\begin{aligned}
\sum \operatorname{sgn}\left(\begin{array}{cccccc}
i_{p-k+1} & \ldots & i_{p} & j_{p-k+1} & \ldots & j_{q} \\
n-q-k+1 & \ldots & n-q & n-q+1 & \ldots & n-p-k
\end{array}\right) r_{n, \ldots, n-p+k+1, i_{p-k+1}, \ldots, i_{p}} \\
\times s_{n, \ldots, n-p+k+1, j_{p-k+1}, \ldots, j_{q}}=0,
\end{aligned}
$$

where the sum is over all index tuples such that the sets $\left\{i_{p-k+1}, \ldots, i_{p}, j_{p-k+1}, \ldots, j_{q}\right\}$ and $\{n-q-k+1, n-q-k+2, \ldots, n-p+k\}$ coincide. We see from the form of the equation that its weight is $\omega_{p-k}^{*}+\omega_{q+k}^{*}$ and the multidegree is $(1,1)$.

Now let us verify that the form $f_{k}$ constructed from the geometric condition for $D_{k}$ specifies a simple divisor. To this end, let us verify that $\operatorname{div} f_{k}$ does not contain the divisors $D_{P, p}, D_{Q, q}, D_{1}, \ldots, D_{k-1}$ and that $f_{k}$ specifies a simple divisor on $U_{k}=X \backslash$ $\left(D_{P, p} \cup D_{Q, q} \cup D_{1} \cup \cdots \cup D_{k-1}\right)$. Then it will follow that $f_{k}$ is proportional to $F_{k}$.

The first assertion follows from the fact that the differences of the weights of $f_{k}$ and of the canonical sections corresponding to these divisors are not dominant weights. The form $f_{k}$ cannot specify a multiple of $D_{k}$, because the multidegree of $f_{k}$ is not a multiple of some other multidegree.

In the other cases of complexity 0, we will omit details of the argument. 
2. $\left(p_{1}, p_{2}\right),\left(1, q_{2}, q_{3}\right)$. Let $p=p_{1}$ and $q=q_{2}$.

(a) $p<1+q$.
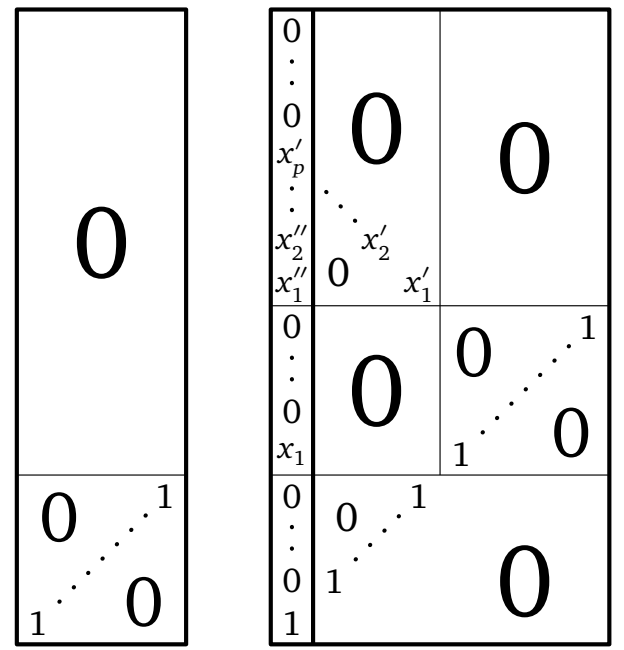

FiguRE $7.2 \mathrm{a}$

\begin{tabular}{|c|c|l|c|}
\hline & deg & \multicolumn{1}{|c|}{ Weight } & Geometric condition \\
\hline$F_{1}$ & $(1,1,0)$ & $\omega_{p+1}^{*}$ & $\left\langle R_{p}+S_{1}\right\rangle \cap E_{n-p-1} \neq 0$ \\
\hline$F_{k}^{\prime}$ & $(1,0,1)$ & $\omega_{p-k}^{*}+\omega_{1+q+k}^{*}$ & $\left\langle R_{p} \cap E_{n-p+k}+S_{1+q} \cap E_{n-p+k}\right\rangle \cap E_{n-(1+q)-k} \neq 0$, \\
& & & $k=1, \ldots, \min (p, n-q-1)$ \\
\hline$F_{k}^{\prime \prime}$ & $(1,1,1)$ & $\omega_{p-k+1}^{*}+\omega_{1+q+k}^{*}$ & $\begin{array}{r}\left\langle\left\langle R_{p}+S_{1}\right\rangle \cap E_{n-p+k-1}+S_{1+q} \cap E_{n-p+k-1}\right\rangle \\
\cap E_{n-(1+q)-k} \neq 0, k=1, \ldots, \min (p-1, n-q-1)\end{array}$ \\
\hline
\end{tabular}

(b) $p=1+q$.

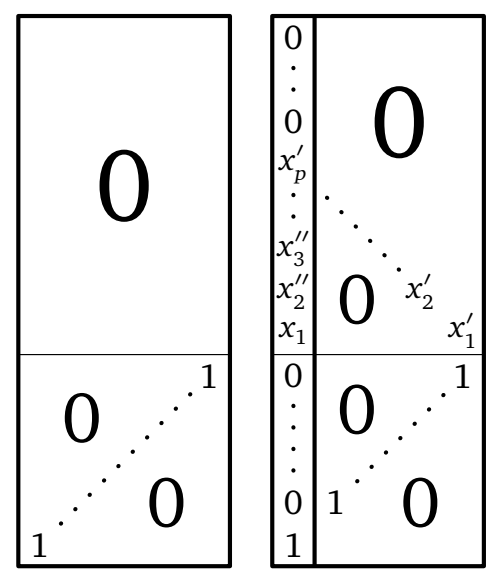

FiguRE $7.2 \mathrm{~b}$ 


\begin{tabular}{|c|c|l|c|}
\hline & $\operatorname{deg}$ & \multicolumn{1}{|c|}{ Weight } & \multicolumn{1}{c|}{ Geometric condition } \\
\hline$F_{1}$ & $(1,1,0)$ & $\omega_{p+1}^{*}$ & $\left\langle R_{p}+S_{1}\right\rangle \cap E_{n-p-1} \neq 0$ \\
\hline$F_{k}^{\prime}$ & $(1,0,1)$ & $\omega_{p-k}^{*}+\omega_{1+q+k}^{*}$ & $\left\langle R_{p} \cap E_{n-p+k}+S_{1+q} \cap E_{n-p+k}\right\rangle \cap E_{n-(1+q)-k} \neq 0$, \\
& & & $k=1, \ldots, \min (p, n-q-1)$ \\
\hline$F_{k}^{\prime \prime}$ & $(1,1,1)$ & $\omega_{p-k+1}^{*}+\omega_{1+q+k}^{*}$ & $\begin{array}{c}\left\langle\left\langle R_{p}+S_{1}\right\rangle \cap E_{n-p+k-1}+S_{1+q} \cap E_{n-p+k-1}\right\rangle \\
\cap E_{n-(1+q)-k} \neq 0, k=2, \ldots, \min (p-1, n-q-1)\end{array}$ \\
& & & \\
\hline
\end{tabular}

(c) $p>1+q$.

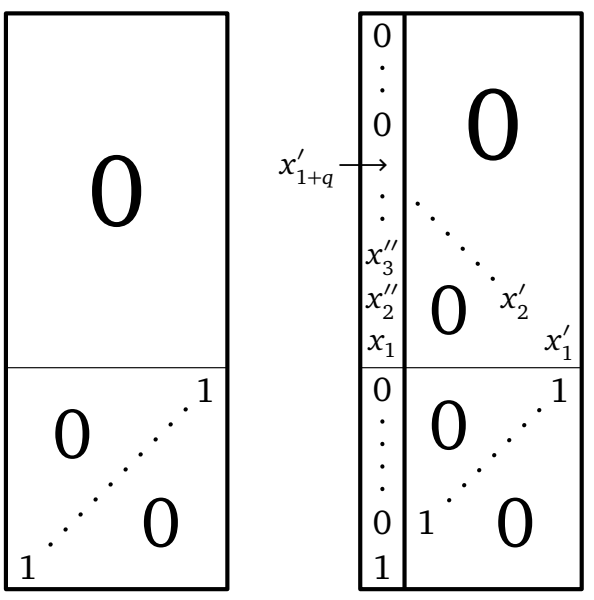

FiguRe $7.2 \mathrm{c}$

\begin{tabular}{|c|c|l|c|}
\hline & deg & Weight & Geometric condition \\
\hline$F_{1}$ & $(1,1,0)$ & $\omega_{p+1}^{*}$ & $\left\langle R_{p}+S_{1}\right\rangle \cap E_{n-p-1} \neq 0$ \\
\hline$F_{k}^{\prime}$ & $(1,0,1)$ & $\omega_{p+k}^{*}+\omega_{1+q-k}^{*}$ & $\begin{array}{r}\left\langle R_{p} \cap E_{n-(1+q)+k}+S_{1+q} \cap E_{n-(1+q)+k}\right\rangle \cap E_{n-p-k} \neq 0, \\
k=1, \ldots, \min (q+1, n-p)\end{array}$ \\
\hline$F_{k}^{\prime \prime}$ & $(1,1,1)$ & $\omega_{p+k}^{*}+\omega_{2+q-k}^{*}$ & $\begin{array}{r}\left\langle\left\langle R_{p}+S_{1}\right\rangle \cap E_{n-(1+q)+k-1}+S_{1+q} \cap E_{n-(1+q)+k-1}\right\rangle \\
\cap E_{n-p-k} \neq 0, k=2, \ldots, \min (q, n-p)\end{array}$ \\
& & & \\
\hline
\end{tabular}

3. $\left(p_{1}, p_{2}\right),\left(q_{1}, 1, q_{3}\right)$. Let $p=p_{1}$ and $q=q_{1}$. We can assume that $p \geq 1+q$.

\begin{tabular}{|c|c|c|r|}
\hline & $\operatorname{deg}$ & Weight & \multicolumn{1}{c|}{ Geometric condition } \\
\hline$F_{k}$ & $(1,1,0)$ & $\omega_{p+k}^{*}+\omega_{q-k}^{*}$ & $\left\langle R_{p} \cap E_{n-p+k}+S_{q} \cap E_{n-p+k}\right\rangle \cap E_{n-p-k} \neq 0$, \\
& & & $k=1, \ldots, \min (q, n-p)$ \\
\hline$F_{k}^{\prime}$ & $(1,0,1)$ & $\omega_{p+k}^{*}+\omega_{q+1-k}^{*}$ & $\left\langle R_{p} \cap E_{n-(q+1)+k}+S_{q+1} \cap E_{n-(q+1)+k}\right\rangle \cap E_{n-p-k} \neq 0$, \\
& & & $k=1, \ldots, \min (q+1, n-p)$ \\
\hline
\end{tabular}



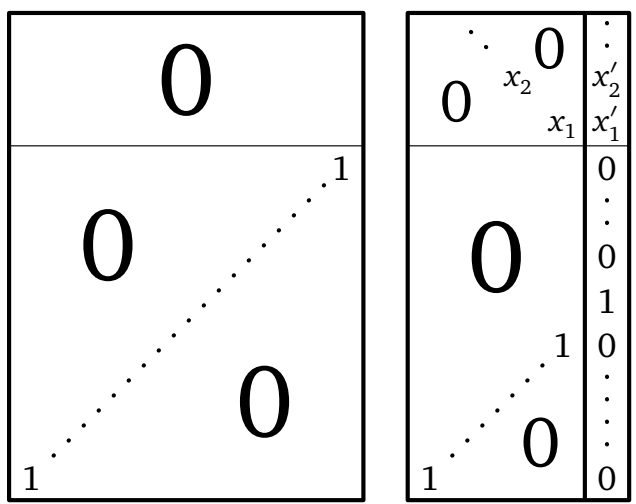

Figure 7.3

4. $(2, p),\left(q_{1}, q_{2}, q_{3}\right)$. We can assume that $q_{1}, q_{2}, q_{3} \geq 2$; otherwise, this is case 2 or 3 .
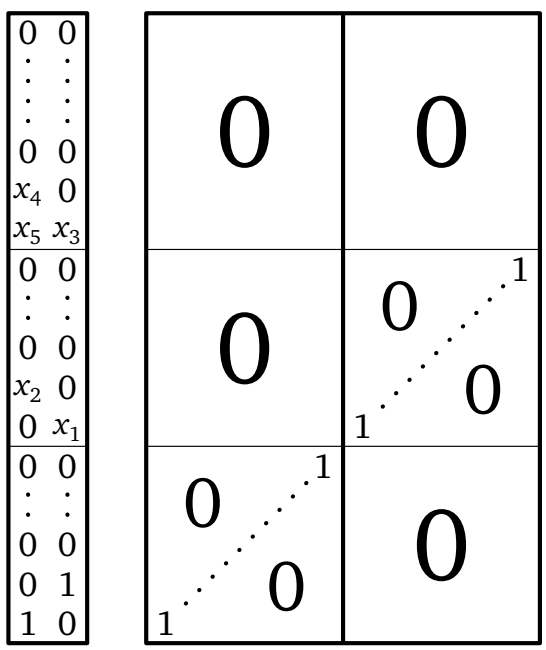

Figure 7.4

\begin{tabular}{|c|c|c|c|}
\hline & $\operatorname{deg}$ & Weight & Geometric condition \\
\hline$F_{k}$ & $(1,1,0)$ & $\omega_{2-k}^{*}+\omega_{q_{1}+k}^{*}$ & $\left\langle R_{2} \cap E_{n-2+k}+S_{q_{1}} \cap E_{n-2+k}\right\rangle \cap E_{n-q_{1}-k} \neq 0$, \\
& & & $k=1,2$ \\
\hline$F_{2+k}$ & $(1,0,1)$ & $\omega_{2-k}^{*}+\omega_{q_{1}+q_{2}+k}^{*}$ & $\left\langle R_{2} \cap E_{n-2+k}+S_{q_{1}+q_{2}} \cap E_{n-2+k}\right\rangle$ \\
$\cap E_{n-\left(q_{1}+q_{2}\right)-k} \neq 0, k=1,2$ \\
\hline$F_{5}$ & $(1,1,1)$ & $\omega_{q_{1}+1}^{*}+\omega_{q_{1}+q_{2}+1}^{*}$ & $\begin{array}{c}\left\langle\left\langle R_{2}+S_{q_{1}}\right\rangle \cap E_{n-q_{1}-1}+S_{q_{1}+q_{2}} \cap E_{n-q_{1}-1}\right\rangle \\
\cap E_{n-\left(q_{1}+q_{2}\right)-1} \neq 0\end{array}$ \\
\hline
\end{tabular}

5. $(1, p),\left(q_{1}, \ldots, q_{s}\right)$. 


\begin{tabular}{|c|c|c|c|}
\hline & $\operatorname{deg}$ & Weight & Geometric condition \\
\hline$F_{k}$ & $(1,0, \ldots, 1, \ldots, 0)$ & $\omega_{q_{1}+\cdots+q_{k}+1}^{*}$ & $\left\langle R_{1}+S_{q_{1}+\cdots+q_{k}}\right\rangle \cap E_{n-\left(q_{1}+\cdots+q_{k}\right)-1} \neq 0$, \\
& & & $k=1, \ldots, s-1$ \\
\hline
\end{tabular}
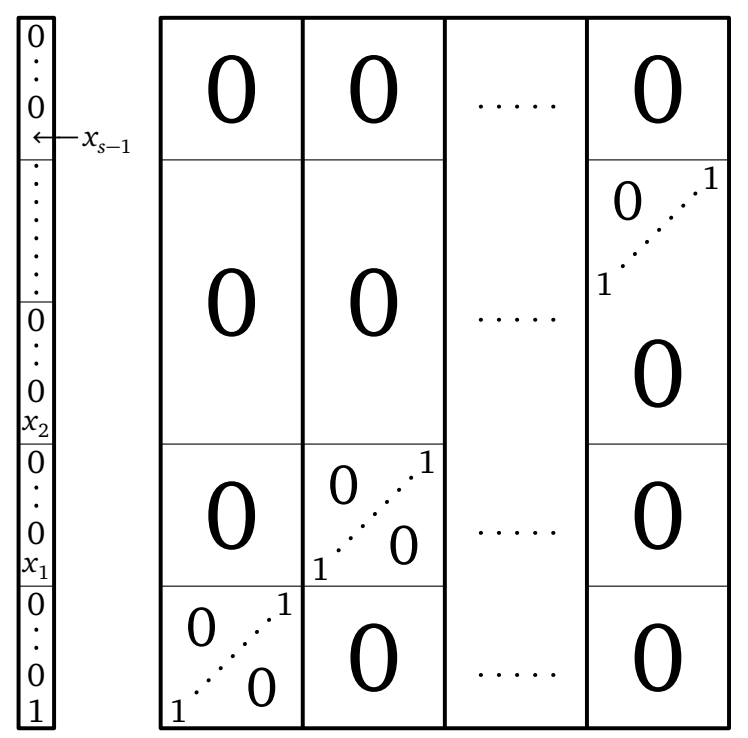

FIGURE 7.5

7.2. Complexity 1.

6. $(2, p),\left(q_{1}, q_{2}, q_{3}, q_{4}\right)$.

(a) $q_{1}, q_{2}, q_{3}, q_{4} \geq 2$.

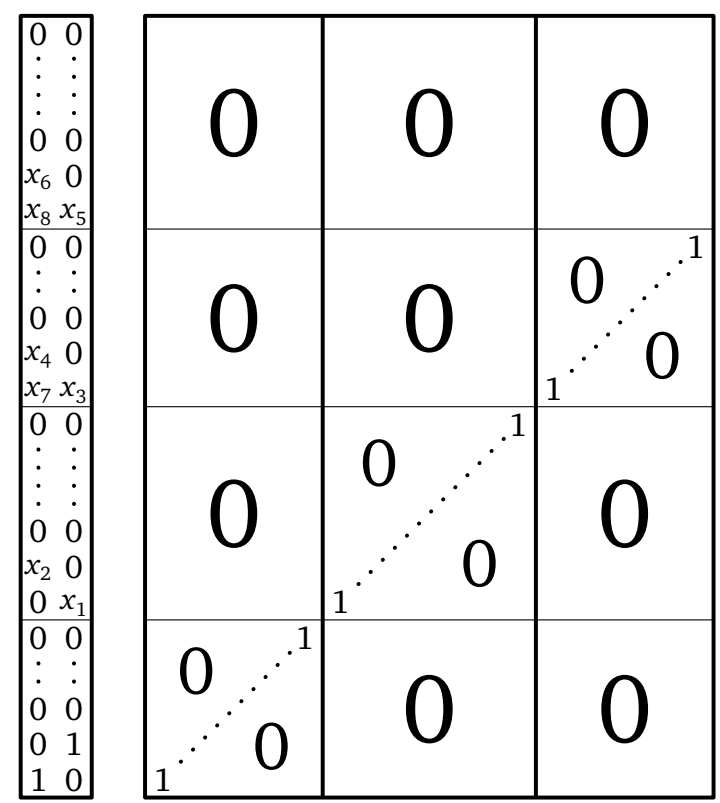

Figure 7.6 


\begin{tabular}{|c|c|c|c|}
\hline & $\operatorname{deg}$ & Weight & Geometric condition \\
\hline$F_{k}$ & $(1,1,0,0)$ & $\omega_{2-k}^{*}+\omega_{q_{1}+k}^{*}$ & $\begin{array}{r}\left\langle R_{2} \cap E_{n-2+k}+S_{q_{1}} \cap E_{n-2+k}\right\rangle \cap E_{n-q_{1}-k} \neq 0, \\
k=1,2\end{array}$ \\
\hline$F_{2+k}$ & $(1,0,1,0)$ & $\omega_{2-k}^{*}+\omega_{q_{1}+q_{2}+k}^{*}$ & $\begin{aligned}\left\langle R_{2} \cap E_{n-2+k}+\right. & \left.S_{q_{1}+q_{2}} \cap E_{n-2+k}\right\rangle \\
& \cap E_{n-\left(q_{1}+q_{2}\right)-k} \neq 0, \quad k=1,2\end{aligned}$ \\
\hline$F_{4+k}$ & $(1,0,0,1)$ & $\omega_{2-k}^{*}+\omega_{q_{1}+q_{2}+q_{3}+k}^{*}$ & $\begin{aligned}\left\langle R_{2} \cap E_{n-2+k}\right. & \left.+S_{q_{1}+q_{2}+q_{3}} \cap E_{n-2+k}\right\rangle \\
& \cap E_{n-\left(q_{1}+q_{2}+q_{3}\right)-k} \neq 0, \quad k=1,2\end{aligned}$ \\
\hline$F_{7}$ & $(1,1,1,0)$ & $\omega_{q_{1}+1}^{*}+\omega_{q_{1}+q_{2}+1}^{*}$ & $\begin{aligned}\left\langle\left(R_{2}+S_{q_{1}}\right) \cap E_{n-q_{1}-1}+S_{q_{1}+q_{2}} \cap E_{n-q_{1}-1}\right\rangle & \cap E_{n-\left(q_{1}+q_{2}\right)-1} \neq 0\end{aligned}$ \\
\hline$F_{8}$ & $(1,1,0,1)$ & $\omega_{q_{1}+1}^{*}+\omega_{q_{1}+q_{2}+q_{3}+1}^{*}$ & $\begin{array}{r}\left\langle\left(R_{2}+S_{q_{1}}\right) \cap E_{n-q_{1}-1}+S_{q_{1}+q_{2}+q_{3}} \cap E_{n-q_{1}-1}\right\rangle \\
\cap E_{n-\left(q_{1}+q_{2}+q_{3}\right)-1} \neq 0\end{array}$ \\
\hline$F_{9}$ & $(1,0,1,1)$ & $\begin{array}{l}\omega_{q_{1}+q_{2}+1}^{*} \\
\quad+\omega_{q_{1}+q_{2}+q_{3}+1}^{*}\end{array}$ & $\begin{aligned} &\left\langle\left\langle R_{2}+S_{q_{1}+q_{2}}\right\rangle \cap E_{n-\left(q_{1}+q_{2}\right)-1}+S_{q_{1}+q_{2}+q_{3}}\right. \\
&\left.\cap E_{n-\left(q_{1}+q_{2}\right)-1}\right\rangle \cap E_{n-\left(q_{1}+q_{2}+q_{3}\right)-1} \neq 0\end{aligned}$ \\
\hline $\begin{array}{l}F_{3} F_{8} \\
F_{5} F_{7} \\
F_{1} F_{9}\end{array}$ & $(2,1,1,1)$ & $\begin{array}{r}\omega_{1}^{*}+\omega_{q_{1}+1}^{*}+\omega_{q_{1}+q_{2}+1}^{*} \\
+\omega_{q_{1}+q_{2}+q_{3}+1}^{*}\end{array}$ & \\
\hline
\end{tabular}

Let us explain how we have found all exceptional divisors and monomials such that a linear relation between them generates the ideal of relations in $R(X)^{U}$. Set $U_{9}=$ $X \backslash\left\{D_{P, 2}, D_{Q, q_{1}}, D_{Q, q_{1}+q_{2}}, D_{Q, q_{1}+q_{2}+q_{3}}, D_{1}, \ldots, D_{8}\right\}$. Then each point in $U_{9}$ can be reduced to a canonical form, i.e., to the form shown in Figure 7.6 where the values of all $x_{i}$ except for $x_{8}$ are equal to 1 ; let $x_{8}=z$. This number $z$ will parametrize the orbits. The orbits in the open set $U_{9}$ are in a one-to-one correspondence with points $z \in \mathbb{C} \backslash\{0\}$. The forms $F_{3} F_{8}$ and $F_{5} F_{7}$ have the same weight and the same degree and can be normalized in such a way that the parameter $z$ can be expressed via these forms as $z=\left(F_{3} F_{8}: F_{5} F_{7}\right)$. The parametric divisors will be given by the forms $F_{3} F_{8}-z F_{5} F_{7}$. Then $z_{D_{5}}=z_{D_{7}}=\infty, z_{D_{3}}=z_{D_{8}}=0$, and $h_{D_{5}}=h_{D_{7}}=h_{D_{3}}=h_{D_{8}}=1$.

Now we should find out what values of $z$ are associated with the exceptional divisors. To this end, it suffices to verify whether the form $F_{3} F_{8}-z F_{5} F_{7}$ is divisible for some values of $z$ by the forms of the remaining divisors in $X \backslash U_{9}$, i.e., by the forms of the divisors in the set $\mathcal{D}_{1}:=\left\{D_{P, 2}, D_{Q, q_{1}}, D_{Q, q_{1}+q_{2}}, D_{Q, q_{1}+q_{2}+q_{3}}, D_{1}, D_{2}, D_{4}, D_{6}\right\}$. The weight of the form $F_{3} F_{8}-z F_{5} F_{7}$ is $\omega_{1}^{*}+\omega_{q_{4}+1}^{*}+\omega_{q_{4}+q_{3}+1}^{*}+\omega_{q_{4}+q_{3}+q_{2}+1}^{*}$, and hence this form, is not divisible by the forms of any divisors in $\mathcal{D}_{1}$ except for $D_{1}$ for any values of $z$ by the weight argument. Let us find a divisor $D_{9}$ such that $F_{1} F_{9}$ has the same weight and degree as the form determining the parametric divisor. By normalizing $F_{1} F_{9}$, we can ensure that $z-1=\left(F_{1} F_{9}: F_{5} F_{7}\right)$. Then the forms thus found satisfy the relation $F_{3} F_{8}-F_{5} F_{7}=F_{1} F_{9}$. We obtain $z_{D_{1}}=z_{D_{9}}=1$ and $h_{D_{1}}=h_{D_{9}}=1$. There exist three exceptional points in this case, 1,0 , and $\infty$. Then the algebra $R(X)^{U}$ is generated by the forms $F_{P, 2}, F_{Q, q_{1}}, F_{Q, q_{1}+q_{2}}, F_{Q, q_{1}+q_{2}+q_{3}}, F_{1}, \ldots, F_{9}$, and the determining relation is $F_{3} F_{8}-F_{5} F_{7}=F_{1} F_{9}$.

In the remaining cases of complexity 1 , we omit the similar argument.

(b) Not all $q_{1}, q_{2}, q_{3}, q_{4}$ are greater than or equal to 2 .

If $q_{1}=1$, then $f_{1}=F_{P, 2} F_{Q, 1}$. Hence $\operatorname{div} f_{1}$ is not simple, and one should delete $F_{1}$ from the generators and replace $F_{1}$ by $F_{P, 2} F_{Q, 1}$ in the relation. 
If $q_{2}=1$, then $f_{7}=F_{2} F_{Q, q_{1}+1}$. Hence $\operatorname{div} f_{7}$ is not simple, and one should delete $F_{7}$ from the generators and replace $F_{7}$ by $F_{2} F_{Q, q_{1}+1}$ in the relation.

If $q_{3}=1$, then $f_{9}=F_{4} F_{Q, q_{1}+q_{2}+1}$. Hence div $f_{9}$ is not simple, and one should delete $F_{9}$ from the generators and replace $F_{9}$ by $F_{4} F_{Q, q_{1}+q_{2}+1}$ in the relation.

If $q_{4}=1$, then the divisor $D_{6}$ is absent.

7. $\left(p_{1}, p_{2}\right),(1,1,1, q), p_{1}, p_{2} \geq 2$. We can assume that $p_{1}, p_{2} \geq 3$. Let $p=p_{1}$.
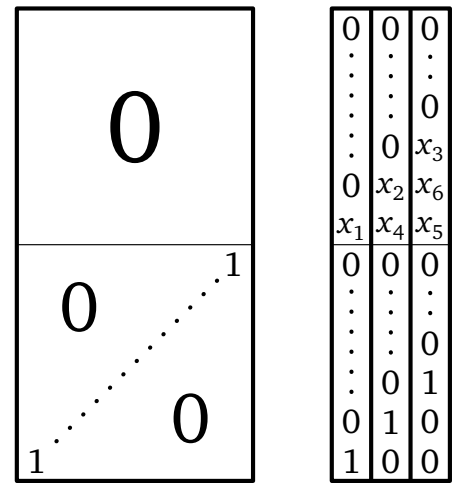

FiguRE 7.7

\begin{tabular}{|c|c|l|c|}
\hline & deg & Weight & Geometric condition \\
\hline$F_{1}$ & $(1,1,0,0)$ & $\omega_{p+1}^{*}$ & $\left\langle R_{p}+S_{1}\right\rangle \cap E_{n-p-1} \neq 0$ \\
\hline$F_{2}$ & $(1,0,1,0)$ & $\omega_{p+2}^{*}$ & $\left\langle R_{p}+S_{2}\right\rangle \cap E_{n-p-2} \neq 0$ \\
\hline
\end{tabular}

\begin{tabular}{|c|c|l|l|}
\hline & deg & \multicolumn{1}{|c|}{ Weight } & \multicolumn{1}{c|}{ Geometric condition } \\
\hline$F_{3}$ & $(1,0,0,1)$ & $\omega_{p+3}^{*}$ & $\left\langle R_{p}+S_{3}\right\rangle \cap E_{n-p-3} \neq 0$ \\
\hline$F_{4}$ & $(1,0,1,0)$ & $\omega_{1}^{*}+\omega_{p+1}^{*}$ & $\left\langle R_{p} \cap E_{n-1}+S_{2} \cap E_{n-1}\right\rangle \cap E_{n-p-1} \neq 0$ \\
\hline$F_{5}$ & $(1,0,0,1)$ & $\omega_{2}^{*}+\omega_{p+1}^{*}$ & $\left\langle R_{p} \cap E_{n-2}+S_{3} \cap E_{n-2}\right\rangle \cap E_{n-p-1} \neq 0$ \\
\hline$F_{6}$ & $(1,1,0,1)$ & $\omega_{2}^{*}+\omega_{p+2}^{*}$ & $\left\langle\left\langle R_{p}+S_{1}\right\rangle \cap E_{n-2}+S_{3} \cap E_{n-2}\right\rangle \cap E_{n-p-2} \neq 0$ \\
\hline$F_{7}$ & $(1,0,0,1)$ & $\omega_{1}^{*}+\omega_{p+2}^{*}$ & $\left\langle R_{p} \cap E_{n-1}+S_{3} \cap E_{n-1}\right\rangle \cap E_{n-p-2} \neq 0$ \\
\hline $\begin{array}{c}F_{Q, 1} F_{2} F_{5} \\
F_{Q, 2} F_{1} F_{7} \\
F_{4} F_{6}\end{array}$ & $(2,1,1,1)$ & $\omega_{1}^{*}+\omega_{2}^{*}$ & \\
\hline
\end{tabular}

8. $\left(p_{1}, p_{2}\right),(1,1, q, 1), p_{1}, p_{2} \geq 2$. We can assume that $p_{1}, p_{2} \geq 3$. Let $p=p_{1}$. 

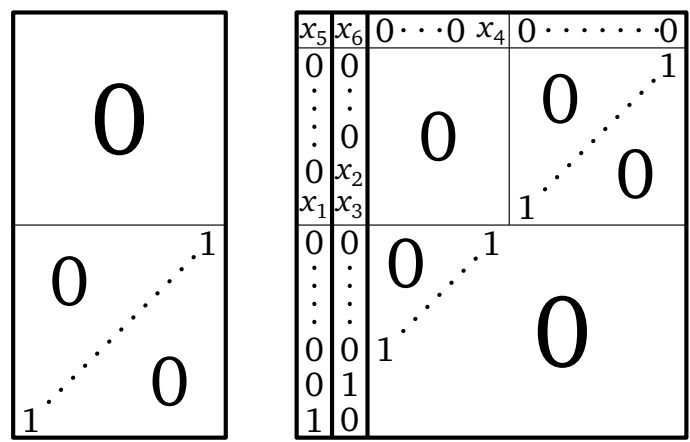

FiguRE 7.8

\begin{tabular}{|c|c|l|l|}
\hline & deg & \multicolumn{1}{|c|}{ Weight } & \multicolumn{1}{c|}{ Geometric condition } \\
\hline$F_{1}$ & $(1,1,0,0)$ & $\omega_{p+1}^{*}$ & $\left\langle R_{p}+S_{1}\right\rangle \cap E_{n-p-1} \neq 0$ \\
\hline$F_{2}$ & $(1,0,1,0)$ & $\omega_{p+2}^{*}$ & $\left\langle R_{p}+S_{2}\right\rangle \cap E_{n-p-2} \neq 0$ \\
\hline$F_{3}$ & $(1,0,1,0)$ & $\omega_{1}^{*}+\omega_{p+1}^{*}$ & $\left\langle R_{p} \cap E_{n-1}+S_{2} \cap E_{n-1}\right\rangle \cap E_{n-p-1} \neq 0$ \\
\hline$F_{4}$ & $(1,0,0,1)$ & $\omega_{p-1}^{*}$ & $\left\langle R_{p} \cap E_{n-p+1}+S_{q+2} \cap E_{n-p+1}\right\rangle \cap E_{0} \neq 0$ \\
\hline$F_{5}$ & $(1,1,0,1)$ & $\omega_{p}^{*}$ & $\left\langle\left\langle R_{p}+S_{1}\right\rangle \cap E_{n-p}+S_{q+2} \cap E_{n-p}\right\rangle \cap E_{0} \neq 0$ \\
\hline$F_{6}$ & $(1,0,1,1)$ & $\omega_{1}^{*}+\omega_{p}^{*}$ & $\left\langle\left\langle R_{p} \cap E_{n-1}+S_{2} \cap E_{n-1}\right\rangle \cap E_{n-p}+S_{q+2}\right.$ \\
\hline$F_{7}$ & $(1,0,1,1)$ & $\omega_{p+1}^{*}$ & \\
\hline$F_{1} F_{6}$ & & & \\
$F_{3} F_{5}$ & $(2,1,1,1)$ & $\omega_{1}^{*}+\omega_{p}^{*}+\omega_{p+1}^{*}$ & \\
$F_{P, p} F_{Q, 1} F_{7}$ & & & \\
\hline
\end{tabular}

9. $(3, p),\left(q_{1}, q_{2}, q_{3}\right), q_{1}, q_{2}, q_{3} \geq 2$.

(a) $q_{1}, q_{2}, q_{3} \geq 3$.
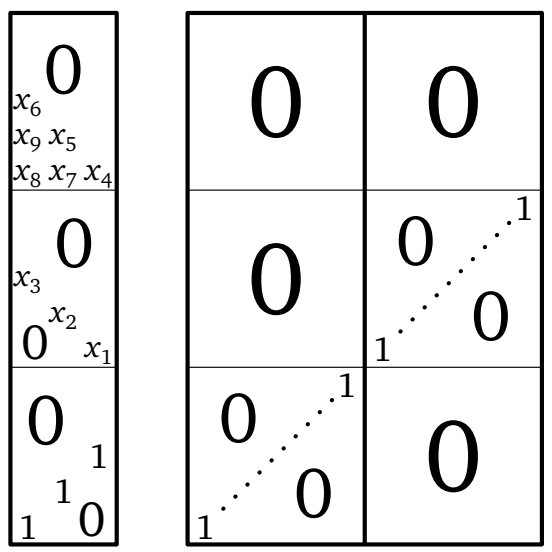

Figure 7.9 


\begin{tabular}{|c|c|c|c|}
\hline & deg & Weight & Geometric condition \\
\hline$F_{k}$ & $(1,1,0)$ & $\omega_{3-k}^{*}+\omega_{q_{1}+k}^{*}$ & $\begin{array}{r}\left\langle R_{3} \cap E_{n-3+k}+S_{q_{1}} \cap E_{n-3+k}\right\rangle \cap E_{n-q_{1}-k} \neq 0 \\
k=1,2,3\end{array}$ \\
\hline$F_{3+k}$ & $(1,0,1)$ & $\omega_{3-k}^{*}+\omega_{q_{1}+q_{2}+k}^{*}$ & $\begin{aligned}\left\langle R_{3} \cap E_{n-3+k}+\right. & \left.S_{q_{1}+q_{2}} \cap E_{n-3+k}\right\rangle \\
& \cap E_{n-\left(q_{1}+q_{2}\right)-k} \neq 0, \quad k=1,2,3\end{aligned}$ \\
\hline$F_{7}$ & $(1,1,1)$ & $\begin{aligned} \omega_{1}^{*}+\omega_{q_{1}+1}^{*} & \\
& +\omega_{q_{1}+q_{2}+1}^{*}\end{aligned}$ & $\begin{aligned}\left\langle\left\langle R_{3} \cap E_{n-1}+S_{q_{1}} \cap E_{n-1}\right\rangle \cap E_{n-q_{1}-1}+S_{q_{1}+q_{2}}\right. \\
\left.\cap E_{n-q_{1}-1}\right\rangle \cap E_{n-\left(q_{1}+q_{2}\right)-1} \neq 0\end{aligned}$ \\
\hline$F_{8}$ & $(1,1,1)$ & $\omega_{q_{1}+2}^{*}+\omega_{q_{1}+q_{2}+1}^{*}$ & $\begin{aligned} &\left\langle\left\langle R_{3}+S_{q_{1}}\right\rangle \cap E_{n-q_{1}-2}+S_{q_{1}+q_{2}} \cap E_{n-q_{1}-2}\right\rangle \\
& \cap E_{n-\left(q_{1}+q_{2}\right)-1} \neq 0\end{aligned}$ \\
\hline$F_{9}$ & $(2,1,1)$ & $\begin{aligned} \omega_{2}^{*}+\omega_{q_{1}+2}^{*} & \\
& +\omega_{q_{1}+q_{2}+2}^{*}\end{aligned}$ & $\begin{array}{l}\left\langle\left\langle R_{3} \cap E_{n-2}+S_{q_{1}+q_{2}} \cap E_{n-2}\right\rangle \cap E_{n-q_{1}-2}\right. \\
\left.\quad+\left\langle R_{3}+S_{q_{1}}\right\rangle \cap E_{n-q_{1}-2}\right\rangle \cap E_{n-\left(q_{1}+q_{2}\right)-2} \neq 0\end{array}$ \\
\hline$F_{10}$ & $(1,1,1)$ & $\omega_{q_{1}+1}^{*}+\omega_{q_{1}+q_{2}+2}^{*}$ & $\begin{aligned}\left\langle\left\langle R_{3}+S_{q_{1}}\right\rangle \cap E_{n-q_{1}-1}+S_{q_{1}+q_{2}} \cap E_{n-q_{1}-1}\right\rangle & \\
\cap E_{n-\left(q_{1}+q_{2}\right)-2} & \neq 0\end{aligned}$ \\
\hline $\begin{array}{c}F_{1} F_{5} F_{8} \\
F_{7} F_{9} \\
F_{2} F_{4} F_{10}\end{array}$ & $(3,2,2)$ & $\begin{array}{l}\omega_{1}^{*}+\omega_{2}^{*}+\omega_{q_{1}+1}^{*}+\omega_{q_{1}+2}^{*} \\
+\omega_{q_{1}+q_{2}+1}^{*}+\omega_{q_{1}+q_{2}+2}^{*}\end{array}$ & \\
\hline
\end{tabular}

(b) Not all $q_{1}, q_{2}, q_{3} \geq 3$.

If $q_{1}=2$, then $f_{1}=F_{P, 3} F_{Q, 2}$. Hence $\operatorname{div} f_{1}$ is not simple, and one should delete $F_{1}$ from the generators and replace $F_{1}$ by $F_{P, 3} F_{Q, 2}$ in the relation.

If $q_{2}=2$, then $f_{8}=F_{3} F_{Q, q_{1}+2}$. Hence $\operatorname{div} f_{8}$ is not simple, and one should delete $F_{8}$ from the generators and replace $F_{8}$ by $F_{3} F_{Q, q_{1}+2}$ in the relation.

If $q_{3}=2$, then the divisor $D_{6}$ is absent.

10. $\left(p_{1}, p_{2}\right),(2,2, q), p_{1}, p_{2} \geq 3$. We can assume that $p_{1}, p_{2} \geq 4$. Let $p=p_{1}$.
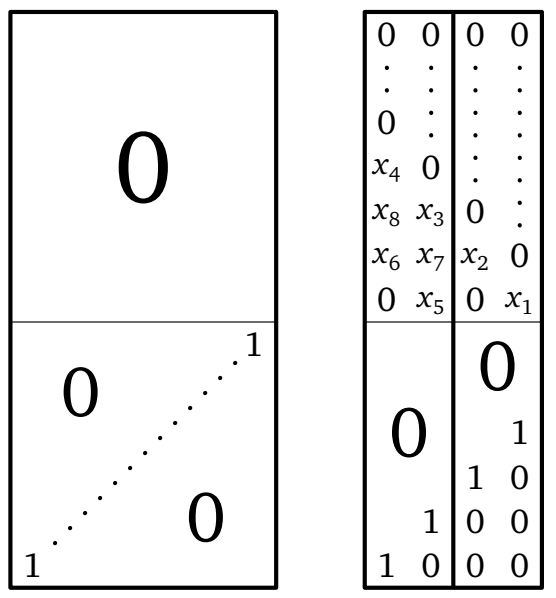

FiguRE 7.10 


\begin{tabular}{|c|c|c|c|}
\hline & $\operatorname{deg}$ & Weight & Geometric condition \\
\hline$F_{k}$ & $(1,0,1)$ & $\omega_{4-k}^{*}+\omega_{p+k}^{*}$ & $\begin{array}{r}\left\langle R_{p} \cap E_{n-4+k}+S_{4} \cap E_{n-4+k}\right\rangle \cap E_{n-p-k} \neq 0, \\
k=1, \ldots, 4\end{array}$ \\
\hline$F_{4+k}$ & $(1,1,0)$ & $\omega_{2-k}^{*}+\omega_{p+k}^{*}$ & $\begin{array}{r}\left\langle R_{p} \cap E_{n-2+k}+S_{2} \cap E_{n-2+k}\right\rangle \cap E_{n-p-k} \neq 0, \\
k=1,2\end{array}$ \\
\hline$F_{7}$ & $(1,1,1)$ & $\omega_{1}^{*}+\omega_{3}^{*}+\omega_{p+2}^{*}$ & $\begin{aligned}\left\langle\left\langle R_{p} \cap E_{n-1}+S_{2} \cap E_{n-1}\right\rangle \cap E_{n-3}+S_{4}\right. \\
\left.\cap E_{n-3}\right\rangle \cap E_{n-p-2} \neq 0\end{aligned}$ \\
\hline$F_{8}$ & $(2,1,1)$ & $\omega_{2}^{*}+\omega_{p+1}^{*}+\omega_{p+3}^{*}$ & $\begin{aligned}\left\langle\left\langle R_{p}+S_{2}\right\rangle\right. & \cap E_{n-p-1}+\left\langle R_{p} \cap E_{n-2}+S_{4}\right. \\
& \left.\left.\cap E_{n-2}\right\rangle \cap E_{n-p-1}\right\rangle \cap E_{n-p-3} \neq 0\end{aligned}$ \\
\hline$F_{9}$ & $(1,1,1)$ & $\omega_{3}^{*}+\omega_{p+3}^{*}$ & $\begin{aligned}\left\langle\left\langle R_{p}+S_{2}\right\rangle \cap E_{n-3}+S_{4} \cap E_{n-3}\right\rangle & \\
& \cap E_{n-p-3} \neq 0\end{aligned}$ \\
\hline $\begin{array}{c}F_{7} F_{8} \\
F_{Q, 2} F_{1} F_{3} F_{6} \\
F_{2} F_{5} F_{9}\end{array}$ & $(3,2,2)$ & $\begin{array}{l}\omega_{1}^{*}+\omega_{2}^{*}+\omega_{3}^{*} \\
+\omega_{p+1}^{*}+\omega_{p+2}^{*}+\omega_{p+3}^{*}\end{array}$ & \\
\hline
\end{tabular}

11. $\left(p_{1}, p_{2}\right),(2, q, 2), p_{1}, p_{2} \geq 3$. We can assume that $p_{1}, p_{2} \geq 4$. Let $p=p_{1}$.
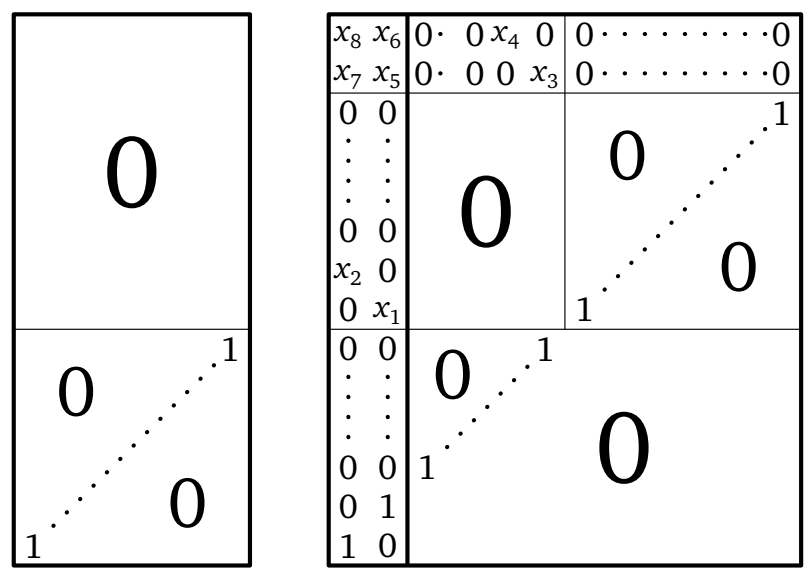

FIGURE 7.11 


\begin{tabular}{|c|c|l|l|}
\hline & deg & Weight & \multicolumn{1}{c|}{ Geometric condition } \\
\hline$F_{k}$ & $(1,1,0)$ & $\omega_{2-k}^{*}+\omega_{p+k}^{*}$ & $\left\langle R_{p} \cap E_{n-2+k}+S_{2} \cap E_{n-2+k}\right\rangle \cap E_{n-p-k} \neq 0$, \\
& & & \\
\hline$F_{2+k}$ & $(1,0,1)$ & $\omega_{p-k}^{*}+\omega_{(q+2)+k}^{*}$ & $\left\langle R_{p} \cap E_{n-p+k}+S_{2+q} \cap E_{n-p+k}\right\rangle \cap E_{n-(2+q)-k} \neq 0$, \\
& & & $k=1,2$ \\
\hline$F_{5}$ & $(1,1,1)$ & $\omega_{1}^{*}+\omega_{p}^{*}+\omega_{n-1}^{*}$ & $\left\langle\left\langle R_{p} \cap E_{n-1}+S_{2} \cap E_{n-1}\right\rangle \cap E_{n-p}+S_{2+q} \cap E_{n-p}\right\rangle$ \\
\hline$F_{6}$ & $(1,1,1)$ & $\omega_{1}^{*}+\omega_{p-1}^{*}$ & $\left\langle\left\langle R_{p} \cap E_{n-1}+S_{2} \cap E_{n-1}\right\rangle \cap E_{n-p+1}+S_{2+q}\right.$ \\
\hline$F_{7}$ & $(1,1,1)$ & $\omega_{p+1}^{*}+\omega_{n-1}^{*}$ & $\left\langle\left\langle R_{p}+S_{2}\right\rangle \cap E_{n-p-1}+S_{2+q} \cap E_{n-p-1}\right\rangle \cap E_{1} \neq 0$ \\
\hline$F_{8}$ & $(2,1,1)$ & $\omega_{p-1}^{*}+\omega_{p+1}^{*}$ & $\left\langle\left\langle R_{p}+S_{2}\right\rangle \cap E_{n-p-1}+\left\langle S_{2+q} \cap E_{n-p+1}+R_{p}\right.\right.$ \\
\hline$F_{9}$ & $(1,1,1)$ & $\omega_{p}^{*}$ & $\left.\left.\cap E_{n-p+1}\right\rangle \cap E_{n-p-1}\right\rangle \cap E_{0} \neq 0$ \\
\hline$F_{P, p} F_{6} F_{7}$ & & & $\langle\langle$ \\
$F_{5} F_{8}$ & $(3,2,2)$ & $\omega_{1}^{*}+\omega_{p-1}^{*}+\omega_{p}^{*}$ & \\
$F_{1} F_{3} F_{9}$ & & $+\omega_{p+1}^{*}+\omega_{n-1}^{*}$ & \\
\hline
\end{tabular}

12. $\left(p_{1}, p_{2}, p_{3}\right),(1,1, q)$.
(a) $p_{1}, p_{2}, p_{3} \geq 2$.
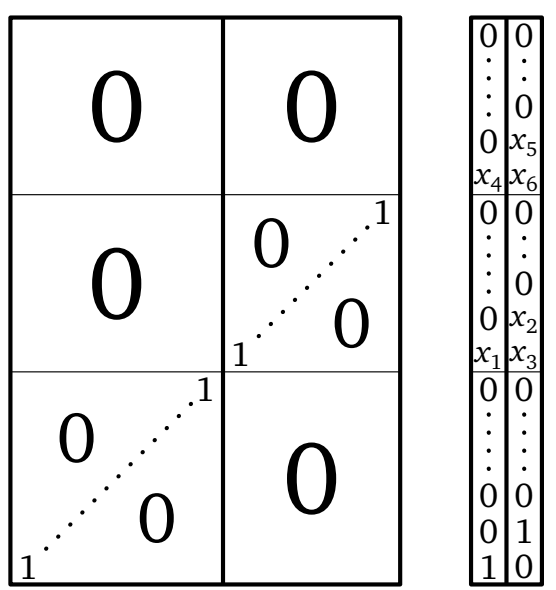

FigURE 7.12 


\begin{tabular}{|c|c|l|l|}
\hline & \multicolumn{1}{|c|}{ deg } & \multicolumn{1}{|c|}{ Weight } & \multicolumn{1}{c|}{ Geometric condition } \\
\hline$F_{1}$ & $(1,0,1,0)$ & $\omega_{p_{1}+1}^{*}$ & $\left\langle R_{p_{1}}+S_{1}\right\rangle \cap E_{n-p_{1}-1} \neq 0$ \\
\hline$F_{2}$ & $(1,0,0,1)$ & $\omega_{p_{1}+2}^{*}$ & $\left\langle R_{p_{1}}+S_{2}\right\rangle \cap E_{n-p_{1}-2} \neq 0$ \\
\hline$F_{3}$ & $(1,0,0,1)$ & $\omega_{1}^{*}+\omega_{p_{1}+1}^{*}$ & $\left\langle R_{p_{1}} \cap E_{n-1}+S_{2} \cap E_{n-1}\right\rangle \cap E_{n-p_{1}-1} \neq 0$ \\
\hline$F_{4}$ & $(0,1,1,0)$ & $\omega_{p_{1}+p_{2}+1}^{*}$ & $\left\langle R_{p_{1}+p_{2}}+S_{1}\right\rangle \cap E_{n-\left(p_{1}+p_{2}\right)-1} \neq 0$ \\
$F_{5}$ & $(0,1,0,1)$ & $\omega_{p_{1}+p_{2}+2}^{*}$ & $\left\langle R_{p_{1}+p_{2}}+S_{2}\right\rangle \cap E_{n-\left(p_{1}+p_{2}\right)-2} \neq 0$ \\
\hline$F_{6}$ & $(0,1,0,1)$ & $\omega_{1}^{*}+\omega_{p_{1}+p_{2}+1}^{*}$ & $\left\langle R_{p_{1}+p_{2}} \cap E_{n-1}+S_{2} \cap E_{n-1}\right\rangle \cap E_{n-\left(p_{1}+p_{2}\right)-1} \neq 0$ \\
\hline$F_{7}$ & $(1,1,0,1)$ & $\omega_{p_{1}+1}^{*}+\omega_{p_{1}+p_{2}+1}^{*}$ & $\left\langle\left\langle R_{p_{1}}+S_{2}\right\rangle \cap E_{n-p_{1}-1}+S_{p_{1}+p_{2}} \cap E_{n-p_{1}-1}\right\rangle$ \\
& & & \\
\hline$F_{3} F_{4}$ & & \multicolumn{2}{|}{} \\
$F_{1} F_{6}$ & $(1,1,1,1)$ & $\omega_{1}^{*}+\omega_{p_{1}+1}^{*}$ & \\
$F_{Q, 1} F_{7}$ & & $+\omega_{p_{1}+p_{2}+1}^{*}$ & \\
\hline
\end{tabular}

(b) Not all $p_{1}, p_{2}, p_{3} \geq 2$.

If $p_{1}=1$, then $f_{3}=F_{P, 1} F_{Q, 2}$. Hence $\operatorname{div} f_{3}$ is not simple, and one should delete $F_{3}$ from the generators and replace $F_{3}$ by $F_{P, 1} F_{Q, 2}$ in the relation.

If $p_{2}=1$, then $f_{7}=F_{2} F_{P, p_{1}+1}$. Hence $\operatorname{div} f_{7}$ is not simple, and one should delete $F_{7}$ from the generators and replace $F_{7}$ by $F_{2} F_{P, p_{1}+1}$ in the relation.

If $p_{3}=1$, then the divisor $D_{5}$ is absent.

13. $\left(p_{1}, p_{2}, p_{3}\right),(1, q, 1)$.

(a) $p_{1}, p_{2}, p_{3} \geq 2$.
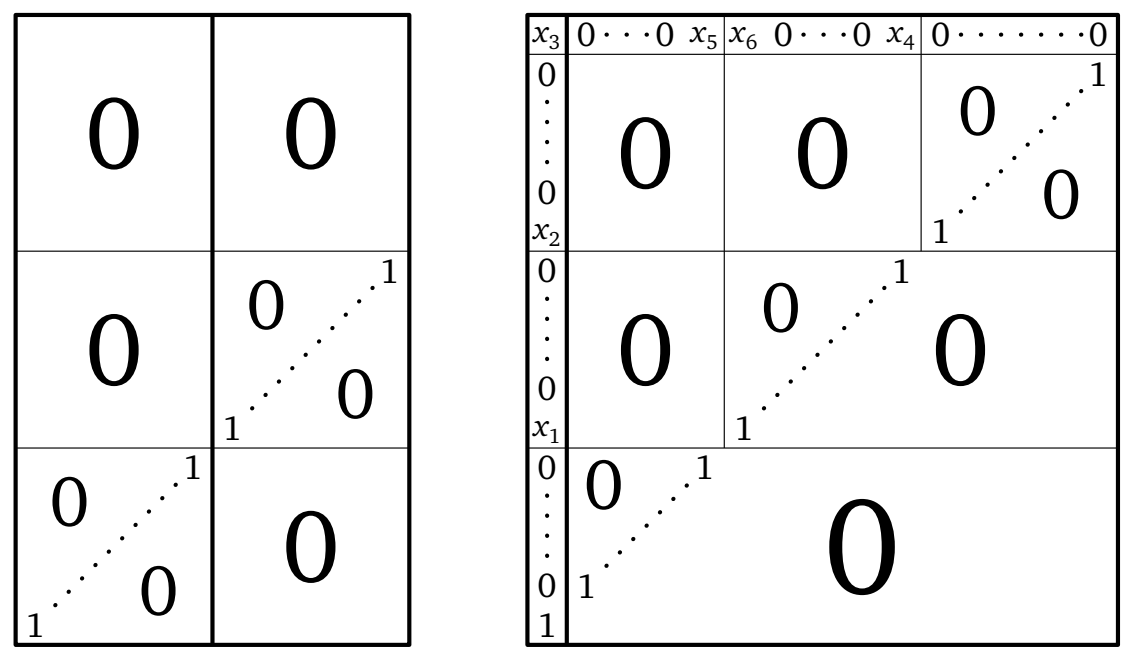

FiguRE 7.13 


\begin{tabular}{|c|c|l|l|}
\hline & deg & \multicolumn{1}{|c|}{ Weight } & \multicolumn{1}{|c|}{ Geometric condition } \\
\hline$F_{1}$ & $(1,0,1,0)$ & $\omega_{p_{1}+1}^{*}$ & $\left\langle R_{p_{1}}+S_{1}\right\rangle \cap E_{n-p_{1}-1} \neq 0$ \\
\hline$F_{2}$ & $(0,1,1,0)$ & $\omega_{p_{1}+p_{2}+1}^{*}$ & $\left\langle R_{p_{1}+p_{2}}+S_{1}\right\rangle \cap E_{n-\left(p_{1}+p_{2}\right)-1} \neq 0$ \\
\hline$F_{3}$ & $(0,1,1,1)$ & $\omega_{p_{1}+p_{2}}^{*}$ & $\left\langle\left\langle R_{p_{1}+p_{2}}+S_{1}\right\rangle \cap E_{n-\left(p_{1}+p_{2}\right)}+S_{1+q} \cap E_{n-\left(p_{1}+p_{2}\right)}\right\rangle$ \\
& & & $\cap E_{0} \neq 0$ \\
\hline$F_{4}$ & $(0,1,0,1)$ & $\omega_{p_{1}+p_{2}-1}^{*}$ & $\left\langle R_{p_{1}+p_{2}} \cap E_{n-\left(p_{1}+p_{2}\right)+1}+S_{1+q} \cap E_{n-\left(p_{1}+p_{2}\right)+1}\right\rangle$ \\
& & & $\cap E_{0} \neq 0$ \\
\hline$F_{5}$ & $(1,0,0,1)$ & $\omega_{p_{1}-1}^{*}$ & $\left\langle R_{p_{1}} \cap E_{n-p_{1}+1}+S_{1+q} \cap E_{n-p_{1}+1}\right\rangle \cap E_{0} \neq 0$ \\
\hline$F_{7}$ & $(1,0,1,1)$ & $\omega_{p_{1}}^{*}$ & $\left\langle\left\langle R_{p_{1}}+S_{1}\right\rangle \cap E_{n-p_{1}}+S_{1+q} \cap E_{n-p_{1}}\right\rangle \cap E_{0} \neq 0$ \\
\hline$F_{P, p_{1}} F_{3}$ & $(1,1,1,1)$ & $\omega_{p_{1}}^{*}+\omega_{p_{1}+p_{2}}^{*}$ & \\
$F_{P, p_{1}+p_{2}} F_{7}$ & & & \\
\hline
\end{tabular}

The divisor $D_{6}$ given in local coordinates by the equation $x_{6}=0$ is parametric.

In this case, we have not specified $D_{6}$ by a geometric condition and hence have slightly deviated from the algorithm described in Section 6. Thus, let us give a brief comment on this. Instead of the divisor given by the condition $x_{6}=0$, we have found the divisor $D_{7}$ given by the condition $x_{6}=1$. The subsequent argument is similar to that in the algorithm except that the orbits in

$$
X \backslash\left(D_{P, p_{1}} \cup D_{P, p_{1}+p_{2}} \cup D_{Q, 1} \cup D_{Q, 1+q} \cup D_{1} \cup \cdots \cup D_{5} \cup D_{7}\right)
$$

are parametrized by numbers $z \in \mathbb{C} \backslash\{1\}$ (rather than $z \in \mathbb{C} \backslash\{0\}$ ).

Note that $R(X)^{U}$ is free in this case.

(b) Not all $p_{1}, p_{2}, p_{3} \geq 2$.

If $p_{1}=1$, then $f_{7}=F_{5} F_{Q, 1}$. Hence $\operatorname{div} f_{7}$ is not simple, and one should delete $F_{7}$ from the generators and replace $F_{7}$ by $F_{5} F_{Q, 1}$ in the product.

If $p_{2}=1$, then there exist three exceptional points, yet another form product $F_{1} F_{4}$ of the same weight and multidegree as $F$ and $F^{\prime}$ occurs, and $R(X)^{U}$ proves to be a hypersurface.

If $p_{3}=1$, then $f_{3}=F_{2} F_{Q, 1+q}$. Hence $\operatorname{div} f_{3}$ is not simple, and one should delete $F_{3}$ from the generators and replace $F_{3}$ by $F_{2} F_{Q, 1+q}$ in the product. 
8. The GROUP $\mathrm{Sp}_{n}$

Set $l=\frac{n}{2}$.

\subsection{Complexity 0.}

1. $(p, p),(p, p), p=l$.
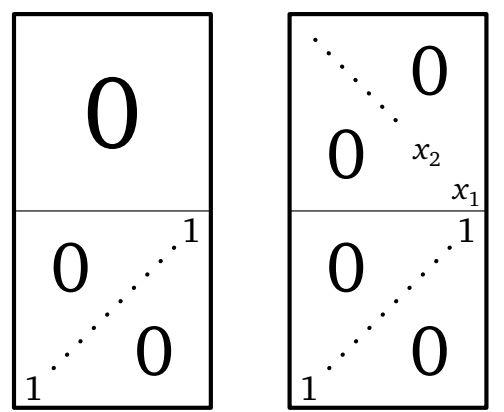

Figure 8.1

\begin{tabular}{|c|c|c|c|}
\hline & $\operatorname{deg}$ & Weight & Geometric condition \\
\hline$F_{k}$ & $(1,1)$ & $2 \omega_{l-k}$ & $\left\langle R_{l} \cap E_{l+k}+S_{l} \cap E_{l+k}\right\rangle \cap E_{l-k} \neq 0, \quad k=1, \ldots, l$ \\
\hline
\end{tabular}

2. $(p, p),(1, q, 1), p=l$.

\begin{tabular}{|l|c|l|l|}
\hline & deg & Weight & Geometric condition \\
\hline$F_{1}$ & $(1,1)$ & $\omega_{l-1}$ & $\left\langle R_{l}+S_{1}\right\rangle \cap E_{l-1} \neq 0$ \\
\hline$F_{2}$ & $(1,2)$ & $\omega_{l}$ & $\left\langle R_{l}+S_{1}\right\rangle \cap E_{l} \perp S_{1}$ \\
\hline
\end{tabular}
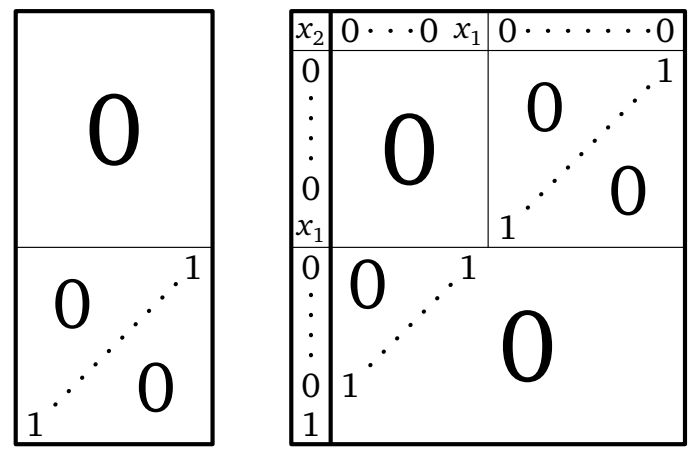

Figure 8.2

3. $(1, p, 1),\left(q_{1}, q_{2}, q_{1}\right)$.

(a) $q_{1}>1$. 

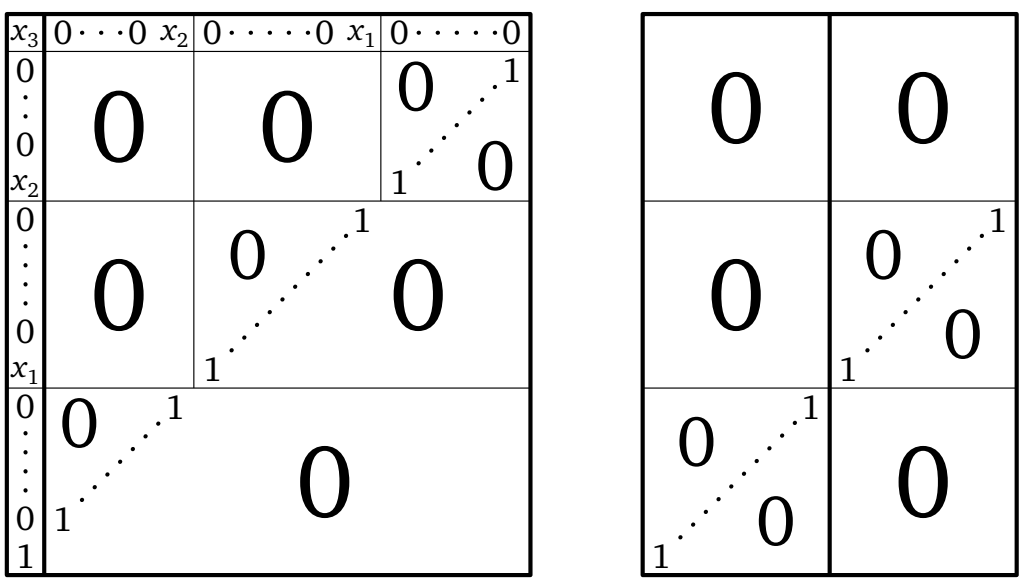

FIGURE 8.3

\begin{tabular}{|c|c|l|c|}
\hline & deg & Weight & Geometric condition \\
\hline$F_{1}$ & $(1,1)$ & $\omega_{q_{1}+1}$ & $\left\langle R_{1}+S_{q_{1}}\right\rangle \cap E_{q_{1}+q_{2}-1} \neq 0$ \\
\hline$F_{2}$ & $(1,1)$ & $\omega_{q_{1}-1}$ & $\left\langle R_{1}+S_{q_{1}+q_{2}}\right\rangle \cap E_{q_{1}-1} \neq 0$ \\
\hline$F_{3}$ & $(2,1)$ & $\omega_{q_{1}}$ & $\left\langle R_{1}+S_{q_{1}+q_{2}}\right\rangle \cap E_{q_{1}} \perp R_{1}$ \\
\hline
\end{tabular}

(b) $q_{1}=1$. In this case, $f_{3}=F_{2} F_{P, 1}$. Hence $\operatorname{div} f_{3}$ is not simple, and one should delete $F_{3}$ from the generators.

\subsection{Complexity 1.}

4. $(p, p),(2, q, 2), p=l$.

(a) $p \geq 4$.
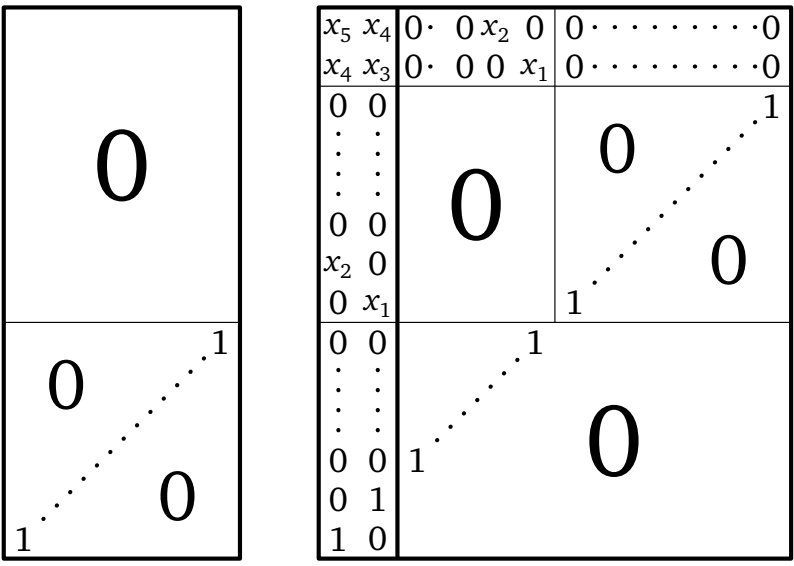

FiguRE 8.4 .1 


\begin{tabular}{|c|c|l|l|}
\hline & deg & \multicolumn{1}{|c|}{ Weight } & \multicolumn{1}{c|}{ Geometric condition } \\
\hline$F_{1}$ & $(1,1)$ & $\omega_{1}+\omega_{l-1}$ & $\left\langle R_{l} \cap E_{n-1}+S_{2} \cap E_{n-1}\right\rangle \cap E_{l-1} \neq 0$ \\
\hline$F_{2}$ & $(1,1)$ & $\omega_{l-2}$ & $\left\langle R_{l}+S_{2}\right\rangle \cap E_{l-2} \neq 0$ \\
\hline$F_{3}$ & $(1,2)$ & $2 \omega_{1}+\omega_{l}$ & $\left\langle R_{l} \cap E_{n-1}+S_{2} \cap E_{n-1}\right\rangle \cap E_{l} \perp S_{2} \cap E_{n-1}$ \\
\hline$F_{4}$ & $(1,2)$ & $\omega_{1}+\omega_{l-1}$ & $\left\langle R_{l}+S_{2}\right\rangle \cap E_{l-1} \perp S_{2} \cap E_{n-1}$ \\
\hline$F_{5}$ & $(2,2)$ & $2 \omega_{l-1}$ & $\left\langle\left\langle R_{l}+S_{2}\right\rangle \cap E_{l-1}+\left\langle R_{l} \cap E_{l+1}+S_{n-2} \cap E_{l+1}\right\rangle \cap E_{l-1}\right\rangle$ \\
\hline$F_{6}$ & $(1,2)$ & $\omega_{l}$ & \\
\hline$F_{3} F_{5}$ & & & $\left\langle\left\langle R_{l}+S_{2}\right\rangle \cap E_{l}+S_{n-2} \cap E_{l}\right\rangle \cap E_{0} \neq 0$ \\
$F_{4}^{2} F_{P, p}$ & $(3,4)$ & $2 \omega_{1}+2 \omega_{l-1}+\omega_{l}$ & \\
$F_{6} F_{1}^{2}$ & & & \\
\hline
\end{tabular}

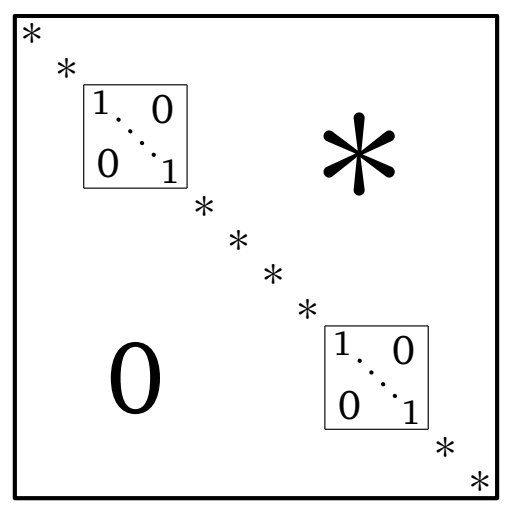

(a) The subgroup $H$

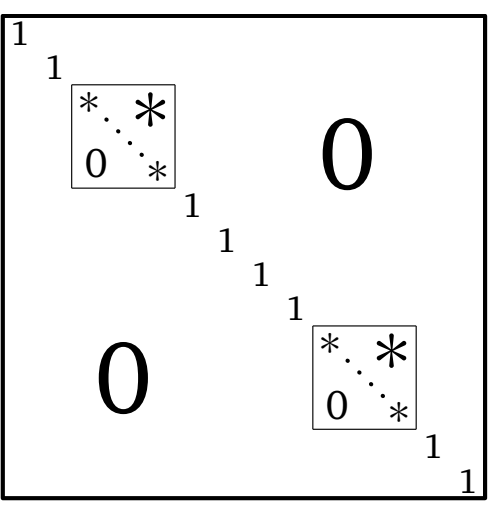

(b) The subgroup $H^{\prime}$

Figure 8.4.2.

Let us show that $\operatorname{div} f_{5}=D_{5}$ rather than $2 D_{5}$ (which could theoretically be possible by the weight-and-degree argument), where $f_{5}$ is the form constructed from the geometric condition for $D_{5}$. To this end, we show that $\operatorname{div} f_{5}$ is simple on the open set $U_{5}=$ $X \backslash\left\{D_{P, p}, D_{Q, 2}, D_{1}, \ldots, D_{4}\right\}$. Each point in $U_{5}$ can be reduced by the action of the group $H \subseteq B$ shown in Figure 8.4.2a to the form shown in Figure 8.4.1, where $x_{1}=$ $\cdots=x_{4}=1$. Consider the section $Z$ consisting of the points of this form. Then $U_{5} \simeq H \times Z$, and the $B$-invariant divisors on $U_{5}$ are the products of $H$ by $H^{\prime}$-invariant divisors on $Z$, where $H^{\prime}$ has the form shown in Figure 8.4.2b. The variable $x_{5}$ is a local coordinate on this section. In these local coordinates, $f_{5}$ is equal to $x_{5}$. Hence $\operatorname{div} f_{5}$ is simple on $U_{5}$.

(b) $p=3$. If $p=3$, then $F_{4}=F_{2} F_{Q, 2}$. Hence $D_{4}$ is not simple, and one should delete $F_{4}$ from the generators and replace $F_{4}$ by $F_{2} F_{Q, 2}$ in the relation.

5. $(1, p, 1),\left(q_{1}, q_{2}, q_{2}, q_{1}\right)$.

(a) $q_{1}, q_{2}>1$. 

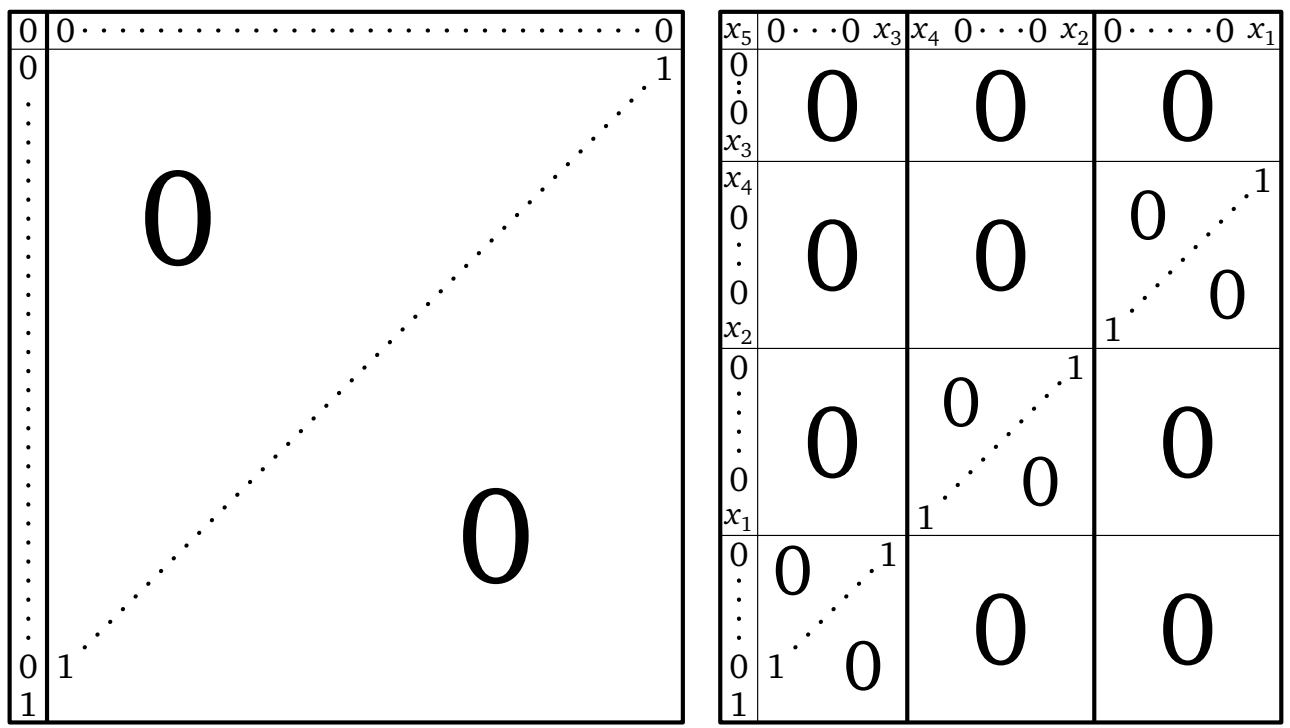

FiguRE 8.5

\begin{tabular}{|c|c|l|c|}
\hline & deg & Weight & Geometric condition \\
\hline$F_{1}$ & $(1,1,0)$ & $\omega_{q_{1}+1}$ & $\left\langle R_{1}+S_{q_{1}}\right\rangle \cap E_{n-q_{1}-1} \neq 0$ \\
\hline$F_{2}$ & $(1,0,1)$ & $\omega_{l-1}$ & $\left\langle R_{1}+S_{l}\right\rangle \cap E_{l-1} \neq 0$ \\
\hline$F_{3}$ & $(1,1,0)$ & $\omega_{q_{1}-1}$ & $\left\langle R_{1}+S_{n-q_{1}}\right\rangle \cap E_{q_{1}-1} \neq 0$ \\
\hline$F_{5}$ & $(2,1,0)$ & $\omega_{q_{1}}$ & $\left\langle R_{1}+S_{q_{1}}\right\rangle \cap E_{n-q_{1}} \perp R_{1}$ \\
\hline$F_{6}$ & $(2,0,1)$ & $\omega_{l}$ & $\left\langle R_{1}+S_{l}\right\rangle \cap E_{l} \perp R_{1}$ \\
\hline $\begin{array}{c}F_{6} F_{Q, q_{1}} \\
F_{5} F_{Q, l}\end{array}$ & $(2,1,1)$ & $\omega_{q_{1}}+\omega_{l}$ & \\
\hline
\end{tabular}

The divisor $D_{4}$ specified by the condition

$$
\left\langle R_{1}+S_{q_{1}}\right\rangle \cap E_{n-q_{1}} \perp\left\langle R_{1}+S_{l}\right\rangle \cap E_{l}
$$

is parametric. (That is why we do not put it in the table.)

Here $R(X)^{U}$ is free.

Let us explain why the form $f_{4}$ constructed from the geometric condition for $D_{4}$ specifies a simple divisor. Note that the multidegree of $F_{4}$ cannot have zero components, because the Schubert divisors and $D_{1}, D_{2}, D_{3}, D_{5}, D_{6}$ exhaust all divisors whose multidegree has zero components. (We know this from cases 2 and 3.) By the weight argument, $\operatorname{div} f_{4}$ does not contain $D_{P, 1}$. Hence $\operatorname{div} f_{4}=D_{4}$.

(b) Not both $q_{1}, q_{2}>1$.

If $q_{1}=1$, then $f_{5}=F_{3} F_{P, 1}$. Hence $\operatorname{div} f_{5}$ is not simple, and one should delete $F_{5}$ from the generators and replace $F_{5}$ by $F_{3} F_{P, 1}$ in the product.

For $q_{1}=1$, the fact that $\operatorname{div} f_{4}$ does not contain $D_{P, 1}$ no longer follows from the weight argument. To verify this fact, let us find a point $x \in D_{P, 1}$ such that $f_{4}(x) \neq 0$. For such a point, we can take $x=\left(R_{1}, S_{1}, S_{l}\right)$, where

$$
R_{1}=\left\langle e_{n-1}\right\rangle, \quad S_{1}=\left\langle e_{n}\right\rangle, \quad S_{l}=\left\langle e_{n}, e_{n-1}+e_{2}, e_{n-2}, \ldots, e_{l+1}\right\rangle .
$$


If $q_{2}=1$, then $f_{4}=F_{1} F_{2}$. Hence there exist three exceptional points, yet another form product $F_{1} F_{2}$ of the same weight and multidegree as $F$ and $F^{\prime}$ occurs, and $R(X)^{U}$ proves to be a hypersurface.

6. $(1, p, 1),\left(q_{1}, q_{2}, q_{3}, q_{2}, q_{1}\right)$.

(a) $q_{1}, q_{2}>1$.
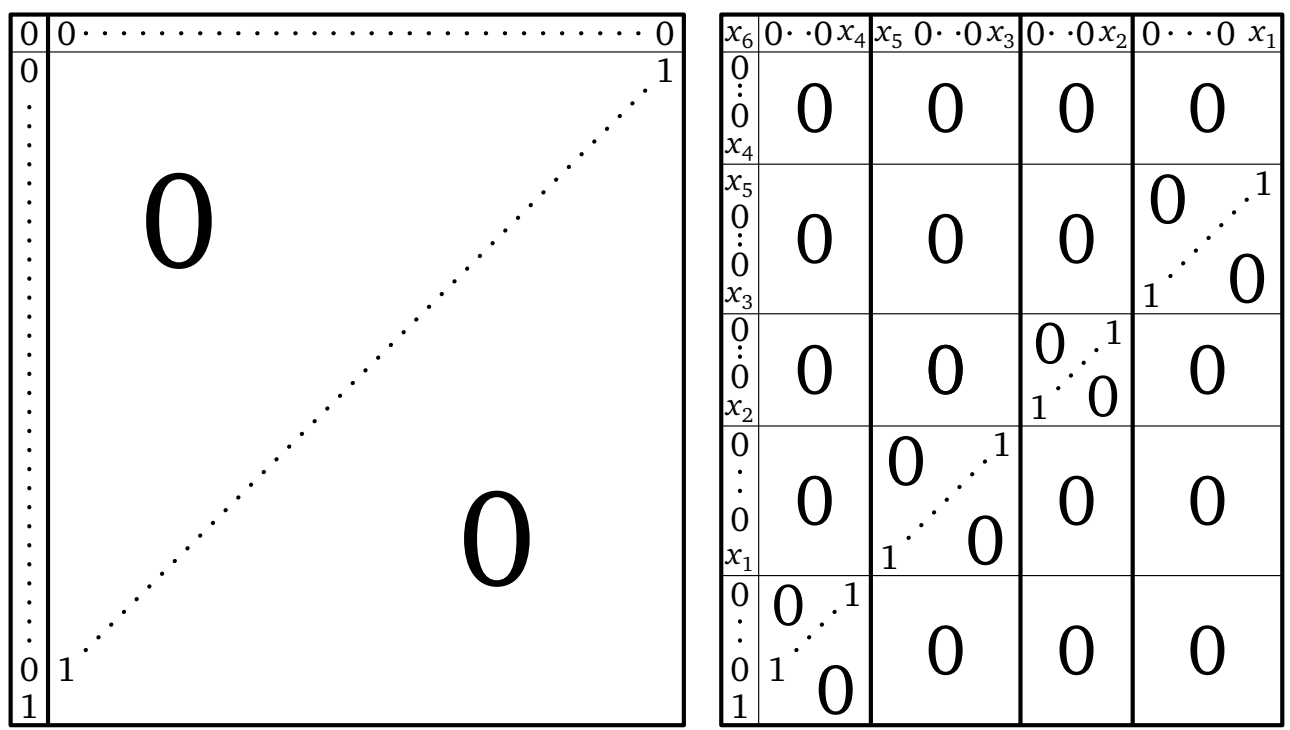

FiguRE 8.6

\begin{tabular}{|c|c|l|c|}
\hline & deg & \multicolumn{1}{|c|}{ Weight } & \multicolumn{1}{c|}{ Geometric condition } \\
\hline$F_{1}$ & $(1,1,0)$ & $\omega_{q_{1}+1}$ & $\left\langle R_{1}+S_{q_{1}}\right\rangle \cap E_{n-q_{1}-1} \neq 0$ \\
\hline$F_{2}$ & $(1,0,1)$ & $\omega_{q_{1}+q_{2}+1}$ & $\left\langle R_{1}+S_{q_{1}+q_{2}}\right\rangle \cap E_{n-\left(q_{1}+q_{2}\right)-1} \neq 0$ \\
\hline$F_{3}$ & $(1,0,1)$ & $\omega_{q_{1}+q_{2}-1}$ & $\left\langle R_{1}+S_{n-\left(q_{1}+q_{2}\right)}\right\rangle \cap E_{q_{1}+q_{2}-1} \neq 0$ \\
\hline$F_{4}$ & $(1,1,0)$ & $\omega_{q_{1}-1}$ & $\left\langle R_{1}+S_{n-q_{1}}\right\rangle \cap E_{q_{1}-1} \neq 0$ \\
\hline$F_{6}$ & $(2,1,0)$ & $\omega_{q_{1}}$ & $\left\langle R_{1}+S_{q_{1}}\right\rangle \cap E_{n-q_{1}} \perp R_{1}$ \\
\hline$F_{7}$ & $(2,0,1)$ & $\omega_{q_{1}+q_{2}}$ & $\left\langle R_{1}+S_{q_{1}+q_{2}}\right\rangle \cap E_{n-\left(q_{1}+q_{2}\right)} \perp R_{1}$ \\
\hline$F_{7} F_{Q, q_{1}}$ & $(2,1,1)$ & $\omega_{q_{1}}+\omega_{q_{1}+q_{2}}$ & \\
$F_{6} F_{Q, q_{1}+q_{2}}$ & & & \\
\hline
\end{tabular}

The divisor $D_{5}$ specified by the condition

$$
\left\langle R_{1}+S_{q_{1}}\right\rangle \cap E_{n-q_{1}} \perp\left\langle R_{1}+S_{q_{1}+q_{2}}\right\rangle \cap E_{n-\left(q_{1}+q_{2}\right)}
$$

is parametric.

Here $R(X)^{U}$ is free.

(b) Not both $q_{1}, q_{2}>1$.

If $q_{1}=1$, then $f_{6}=F_{4} F_{P, 1}$. Hence $\operatorname{div} f_{6}$ is not simple, and one should delete $F_{6}$ from the generators and replace $F_{6}$ by $F_{4} F_{P, 1}$ in the product. 
If $q_{2}=1$, then $f_{5}=F_{1} F_{3}$. Hence there exist three exceptional points, yet another form product $F_{1} F_{3}$ of the same weight and multidegree as $F$ and $F^{\prime}$ occurs, and $R(X)^{U}$ proves to be a hypersurface.

7. $(2,2),(1,1,1,1)$.

$$
\begin{array}{|ll}
\hline 0 & 0 \\
0 & 0 \\
0 & 1 \\
1 & 0
\end{array} \quad \begin{array}{c|c|c|c|}
x_{3} & x_{1} & 0 \\
x_{1} & x_{2} & 1 \\
0 & 1 & 0 \\
1 & 0 & 0 \\
\hline
\end{array}
$$

FiguRE 8.7

\begin{tabular}{|c|c|c|c|}
\hline & $\operatorname{deg}$ & Weight & Geometric condition \\
\hline$F_{1}$ & $(1,1,0)$ & $\omega_{1}$ & $\left\langle R_{2}+S_{1}\right\rangle \cap E_{1} \neq 0$ \\
\hline$F_{2}$ & $(1,0,1)$ & $2 \omega_{1}$ & $\left\langle R_{2} \cap E_{3}+S_{2} \cap E_{3}\right\rangle \cap E_{1} \neq 0$ \\
\hline$F_{3}$ & $(1,2,0)$ & $\omega_{2}$ & $\left\langle R_{2}+S_{1}\right\rangle \cap E_{2} \perp S_{1}$ \\
\hline$F_{4}$ & $(1,0,1)$ & 0 & $\left\langle R_{2}+S_{2}\right\rangle \cap E_{0} \neq 0$ \\
\hline $\begin{array}{c}F_{2} F_{3} \\
F_{1}^{2} F_{Q, 2} \\
F_{4} F_{P, 2} F_{Q, 1}^{2}\end{array}$ & $(2,1,1)$ & $2 \omega_{1}+\omega_{2}$ & \\
\hline
\end{tabular}

9. THE GROUP $\mathrm{SO}_{n}$

Set $l=\left[\frac{n}{2}\right]$.

\subsection{Complexity 0.}

1. $(p, p),(p, p), p=l$.
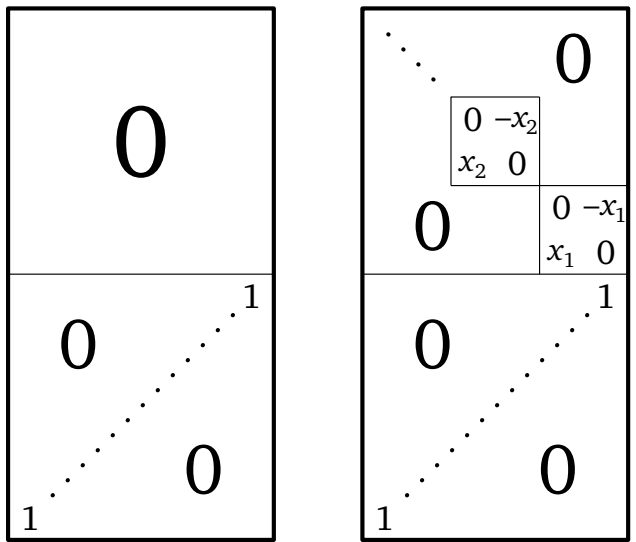

Figure 9.1.1 


\begin{tabular}{|c|l|l|c|}
\hline & \multicolumn{1}{|c|}{$\operatorname{deg}$} & Weight & Geometric condition \\
\hline$F_{k}^{2}$ & $(1,1)_{P l}$ & $2 \pi_{l-2 k}$ & $\left\langle R_{l} \cap E_{l+2 k}+S_{l} \cap E_{l+2 k}\right\rangle \cap E_{l-2 k} \neq 0, k=1, \ldots,[l / 2]$ \\
$F_{k}$ & $(1,1)$ & $\omega_{l-2 k}$ & \\
\hline
\end{tabular}

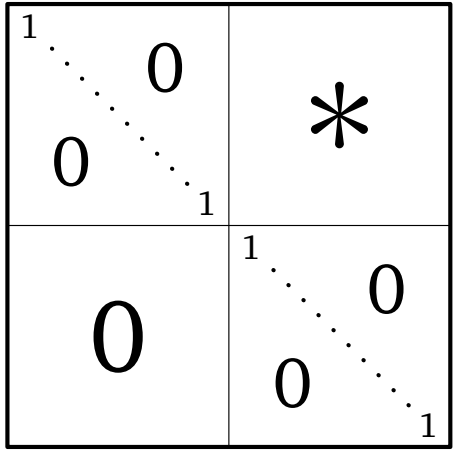

(a) The subgroup $H_{1}$

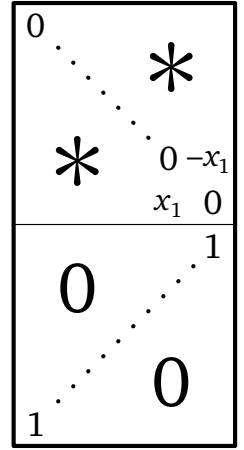

(b) The subspace $S_{p}$

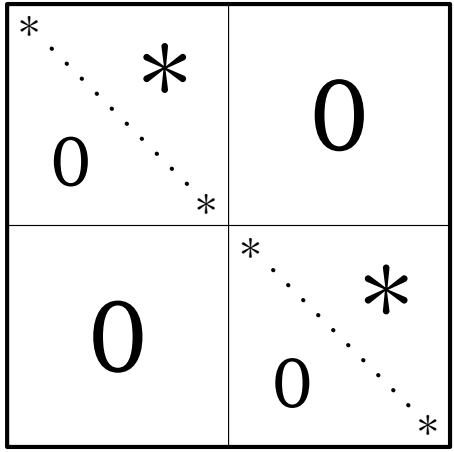

(c) The subgroup $H_{1}^{\prime}$

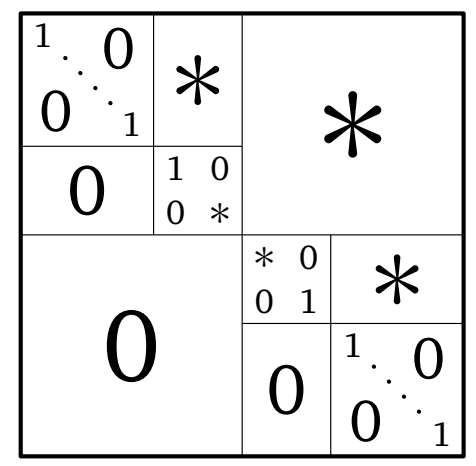

(d) The subgroup $\mathrm{H}_{2}$

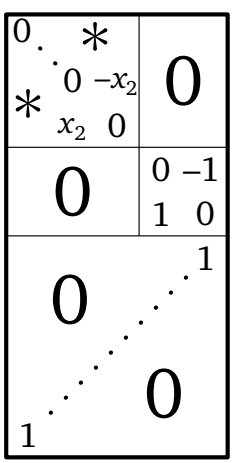

(e) The subspace $S_{p}$

Figure 9.1.2.

Let us explain why the forms obtained from the geometric conditions written out above specify double divisors. We will use the subgroup $H_{1}$ (see Figure 9.1.2a), which is smaller than $B$. Then the subspace $R_{p}$ can be reduced outside $D_{P, p}$ and $D_{Q, p}$ to the form shown in Figure 9.1.1 on the left, and the subspace $S_{p}$ can be reduced to the form shown in Figure 9.1.2b, Consider the section $Z$ consisting of the points of this form. Then $U_{1}:=X \backslash\left\{D_{P, p}, D_{Q, p}\right\} \simeq H_{1} \times Z$. Note that $B=H_{1} H_{1}^{\prime}$, where the subgroup $H_{1}^{\prime}$ is shown in Figure $9.1 .2 \mathrm{C}$. The $B$-invariant divisors on $U_{1}$ are the products of $H_{1}$ by $H_{1}^{\prime}$-invariant divisors on $Z$. The variable $x_{1}$ and the elements shown by asterisks in Figure 9.1.2b are local coordinates on the section $Z$. In these local coordinates, the form corresponding to the geometric condition for $k=1$ can be written as $x_{1}^{2}$. Hence this form specifies the double divisor $2 D_{1}$.

We proceed further in a similar way. Consider a point in $U_{1}:=X \backslash\left\{D_{P, p}, D_{Q, p}, D_{1}\right\}$. By using the action of the group $H_{2}$ (see Figure 9.1.2d), one can reduce the point to the form shown in Figure 9.1.2e In the local coordinates, the form corresponding to the geometric condition for $k=2$ is $x_{2}^{2}$ and hence specifies $2 D_{2}$. The argument for the remaining $k$ is similar. 
2. $(p, p),(p, p)^{\prime}, p=l$.
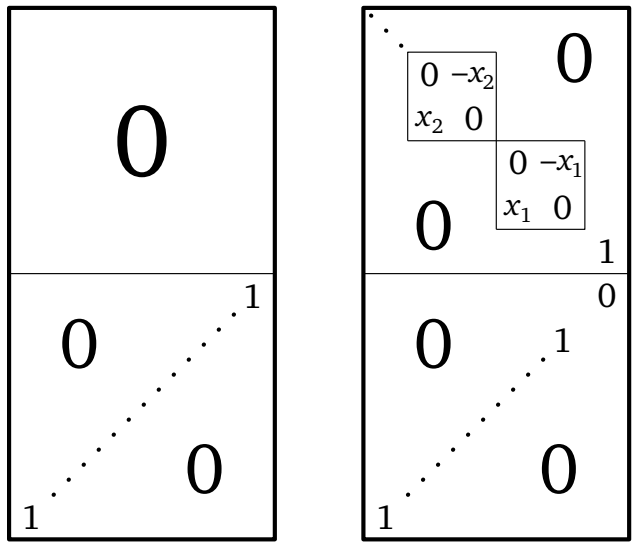

FiguRE 9.2

\begin{tabular}{|l|l|l|r|}
\hline & \multicolumn{1}{|c|}{$\operatorname{deg}$} & Weight & \multicolumn{1}{|c|}{ Geometric condition } \\
\hline$F_{k}^{2}$ & $(1,1)_{P l}$ & $2 \pi_{l-1-2 k}$ & $\left\langle R_{l} \cap E_{l+1+2 k}+S_{l}^{\prime} \cap E_{l+1+2 k}\right\rangle \cap E_{l-1-2 k} \neq 0$, \\
$F_{k}$ & $(1,1)$ & $\omega_{l-1-2 k}$ & $k=1, \ldots,[(l-1) / 2]$ \\
\hline
\end{tabular}

3. $(p, p),(1, q, 1), p=l$.
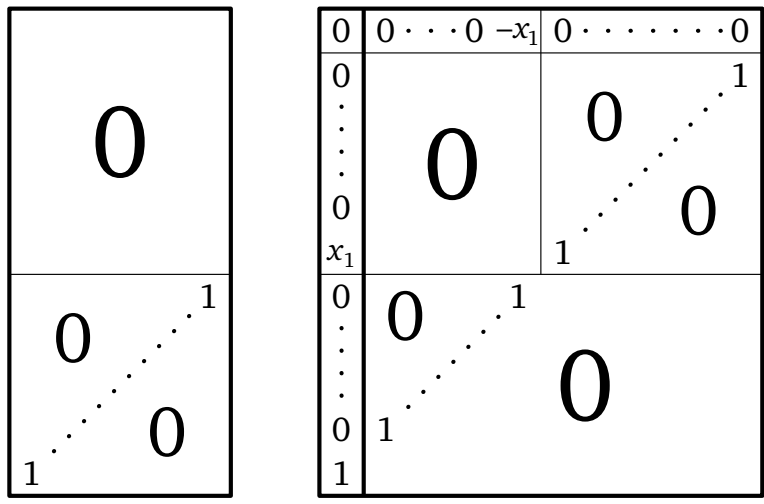

Figure 9.3

\begin{tabular}{|c|l|l|l|}
\hline & \multicolumn{1}{|c|}{$\operatorname{deg}$} & Weight & Geometric condition \\
\hline$F_{1}^{2}$ & $(1,2)_{P l}$ & $\pi_{l}^{\prime}$ & $\left\langle R_{l}+S_{1}\right\rangle \cap E_{l}^{\prime} \perp S_{1}$ \\
$F_{1}$ & $(1,1)$ & $\omega_{l-1}$ & \\
\hline
\end{tabular}


4. $(p, p),(2, q, 2), p=l$.

(a) $p \geq 4$.
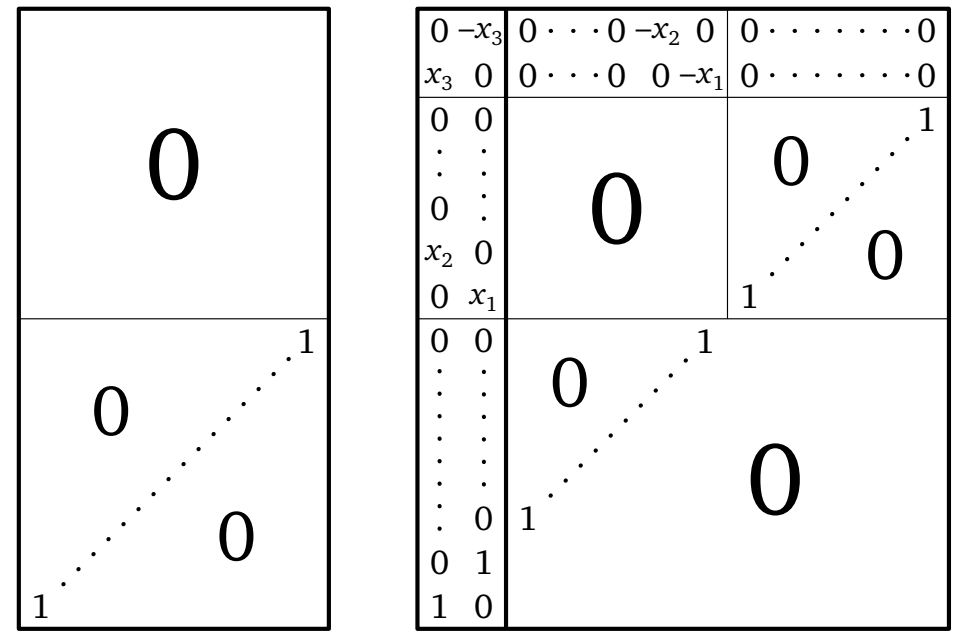

FiguRE 9.4

\begin{tabular}{|l|l|l|c|}
\hline & \multicolumn{1}{|c|}{$\operatorname{deg}$} & \multicolumn{1}{|c|}{ Weight } & \multicolumn{1}{|c|}{ Geometric condition } \\
\hline$F_{1}^{2}$ & $(1,2)_{P l}$ & $2 \pi_{1}+\pi_{l}^{\prime}$ & $\left\langle R_{l} \cap E_{n-1}+S_{2} \cap E_{n-1}\right\rangle \cap E_{l}^{\prime} \perp S_{2} \cap E_{n-1}$ \\
$F_{1}$ & $(1,1)$ & $\omega_{1}+\omega_{l-1}$ & \\
\hline$F_{2}$ & $(1,1)_{P l}$ & $\pi_{l-2}$ & $\left\langle R_{l}+S_{2}\right\rangle \cap E_{l-2} \neq 0$ \\
$F_{2}$ & $(2,1)$ & $\omega_{l-2}$ & \\
\hline$F_{3}^{2}$ & $(1,2)_{P l}$ & $\pi_{l}$ & $\left\langle\left\langle R_{l}+S_{2}\right\rangle \cap E_{l}+S_{n-2} \cap E_{l}\right\rangle \cap E_{0} \neq 0$ \\
$F_{3}$ & $(1,1)$ & $\omega_{l}$ & \\
\hline
\end{tabular}

(b) $p=3$.

We can drop this case, because $\mathrm{SO}_{6}$ is locally isomorphic to $\mathrm{SL}_{4}$. 
5. $(p, p),(3, q, 3), p=l$.

(a) $p \geq 6$.
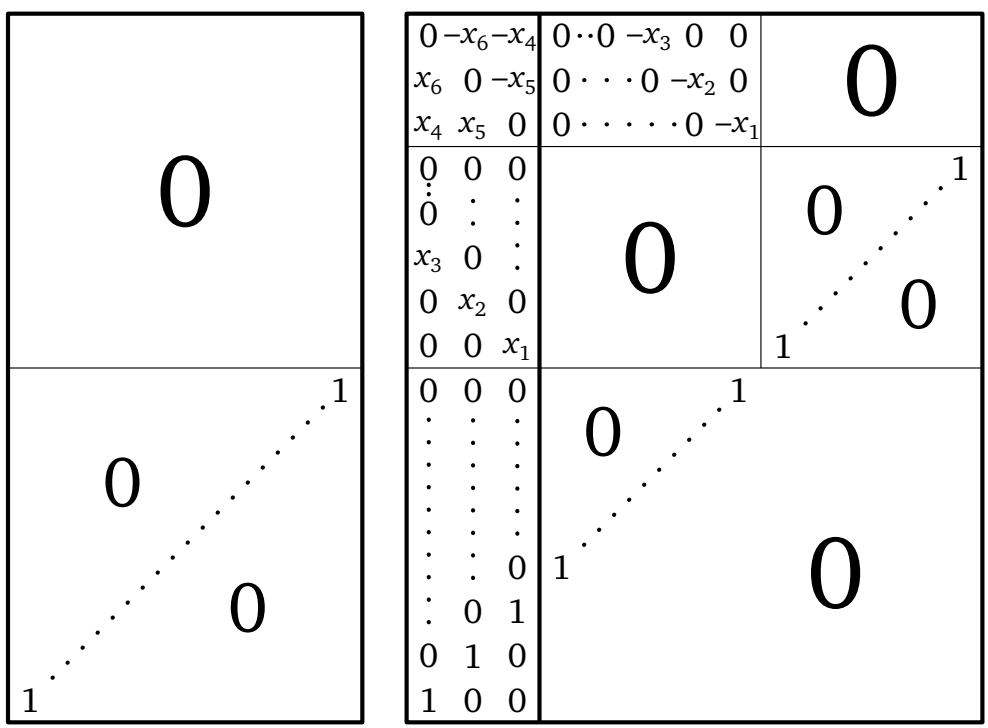

FIGURE 9.5

\begin{tabular}{|l|l|l|l|}
\hline & \multicolumn{1}{|c|}{ deg } & \multicolumn{1}{|c|}{ Weight } & Geometric condition \\
\hline$F_{1}^{2}$ & $(1,2)_{P l}$ & $2 \pi_{2}+\pi_{l}^{\prime}$ & $\left\langle R_{l} \cap E_{n-2}+S_{3} \cap E_{n-2}\right\rangle \cap E_{l}^{\prime} \perp S_{3} \cap E_{n-2}$ \\
$F_{1}$ & $(1,1)$ & $\omega_{2}+\omega_{l-1}$ & \\
\hline$F_{2}$ & $(1,1)_{P l}$ & $\pi_{1}+\pi_{l-2}$ & $\left\langle R_{l} \cap E_{n-1}+S_{3} \cap E_{n-1}\right\rangle \cap E_{l-2} \neq 0$ \\
$F_{2}$ & $(2,1)$ & $\omega_{1}+\omega_{l-2}$ & \\
\hline$F_{3}$ & $(1,1)_{P l}$ & $\pi_{l-3}$ & $\left\langle R_{l}+S_{3}\right\rangle \cap E_{l-3} \neq 0$ \\
$F_{3}$ & $(2,1)$ & $\omega_{l-3}$ & \\
$F_{4}$ & $(1,2)_{P l}$ & $\pi_{2}+\pi_{l-2}$ & $\left\langle R_{l}+S_{3}\right\rangle \cap E_{l-2} \perp S_{3} \cap E_{n-2}$ \\
$F_{4}$ & $(2,2)$ & $\omega_{2}+\omega_{l-2}$ & \\
\hline$F_{5}^{2}$ & $(1,2)_{P l}$ & $2 \pi_{1}+\pi_{l}$ & $\left\langle\left\langle R_{l} \cap E_{n-1}+S_{3} \cap E_{n-1}\right\rangle \cap E_{l}+S_{n-3} \cap E_{l}\right\rangle \cap E_{1} \neq 0$ \\
$F_{5}$ & $(1,1)$ & $\omega_{1}+\omega_{l}$ & \\
\hline$F_{6}^{2}$ & $(1,2)_{P l}$ & $\pi_{l}^{\prime}$ & $\left\langle\left\langle R_{l}+S_{3}\right\rangle \cap E_{l}^{\prime}+S_{n-3} \cap E_{l}^{\prime}\right\rangle \cap E_{0} \neq 0$ \\
$F_{6}$ & $(1,1)$ & $\omega_{l-1}$ & \\
\hline
\end{tabular}

(b) $p=5$. In this case, $f_{4}=F_{3} F_{Q, 3}$. Hence $\operatorname{div} f_{4}$ is not simple, and one should delete $F_{4}$ from the generators.

(c) $p=4$. This is a special case of case 6 . 
6. $(p, p),(q, 2, q), p=l$.
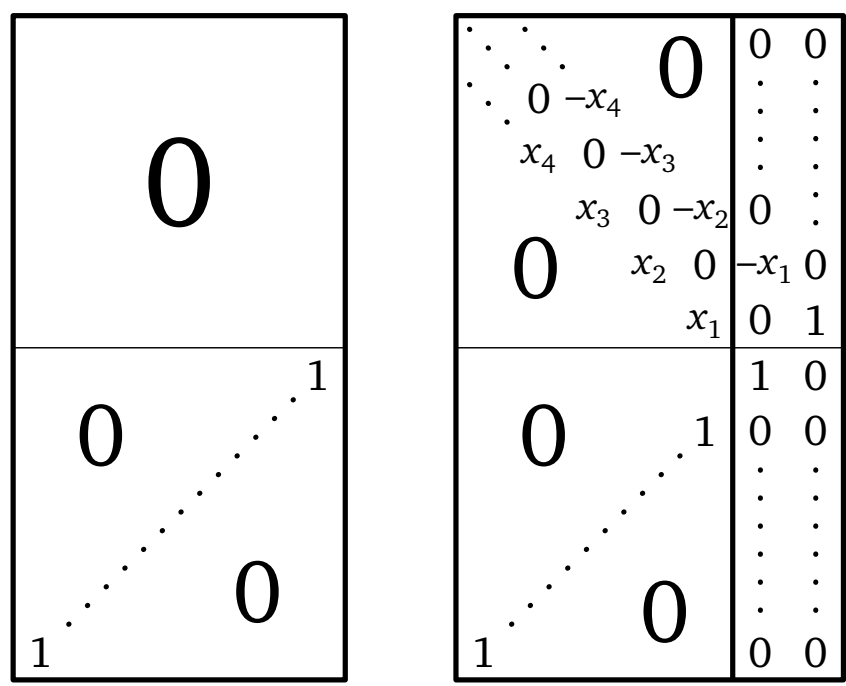

FIGURE 9.6

\begin{tabular}{|l|l|l|r|}
\hline & \multicolumn{1}{|c|}{$\operatorname{deg}$} & \multicolumn{1}{|c|}{ Weight } & \multicolumn{1}{|c|}{ Geometric condition } \\
\hline$F_{2 k-1}^{2}$ & $(1,0,1,0)_{P l}$ & $2 \pi_{l-2 k}$ & $\left\langle R_{l} \cap E_{l+2 k}+S_{l} \cap E_{l+2 k}\right\rangle \cap E_{l-2 k} \neq 0$, \\
$F_{2 k-1}$ & $(1,0,1)$ & $\omega_{l-2 k}$ & $k=1, \ldots,[l / 2]$ \\
\hline$F_{2 k}^{2}$ & $(1,0,0,1)_{P l}$ & $2 \pi_{l-2 k-1}$ & $\left\langle R_{l} \cap E_{l+2 k+1}+S_{l}^{\prime} \cap E_{l+2 k+1}\right\rangle \cap E_{l-2 k-1} \neq 0$, \\
$F_{2 k}$ & $(1,1,0)$ & $\omega_{l-2 k-1}$ & $k=1, \ldots,[(l-1) / 2]$ \\
\hline
\end{tabular}

7. $(p, p),(1, q, q, 1), p=l$.

\begin{tabular}{|c|l|l|r|}
\hline & \multicolumn{1}{|c|}{$\operatorname{deg}$} & \multicolumn{1}{|c|}{ Weight } & Geometric condition \\
\hline$F_{k}^{2}$ & $(1,0,1)_{P l}$ & $2 \pi_{l-2 k}$ & $\left\langle R_{l} \cap E_{l+2 k}+S_{l} \cap E_{l+2 k}\right\rangle \cap E_{l-2 k} \neq 0, k=1, \ldots,[l / 2]$ \\
$F_{k}$ & $(1,0,1)$ & $\omega_{l-2 k}$ & \\
\hline$F_{1}^{\prime 2}$ & $(1,1,0)_{P l}$ & $\pi_{l}^{\prime}$ & $\left\langle R_{l}+S_{1}\right\rangle \cap E_{l}^{\prime} \perp S_{1}$ \\
$F_{1}^{\prime}$ & $(1,1,0)$ & $\omega_{l-1}$ & $k=2, \ldots,[(l-1) / 2]$ \\
\hline$F_{k}^{\prime 2}$ & $(1,2,1)_{P l}$ & $2 \pi_{l-2 k+1}$ & $\left\langle\left\langle R_{l}+S_{1}\right\rangle \cap E_{l+2 k-1}+S_{l} \cap E_{l+2 k-1}\right\rangle \cap E_{l-2 k+1} \perp S_{1}$, \\
$F_{k}^{\prime}$ & $(1,1,1)$ & $\omega_{l-2 k+1}$ & $k=2$ \\
\hline
\end{tabular}



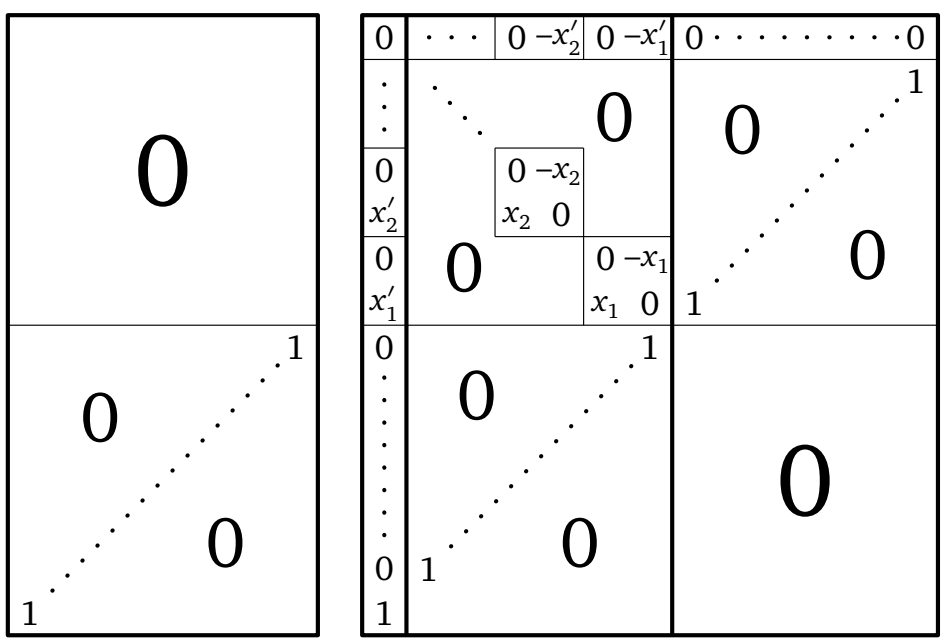

FIGURE 9.7

8. $(p, p),(1, q, q, 1)^{\prime}, p=l$.
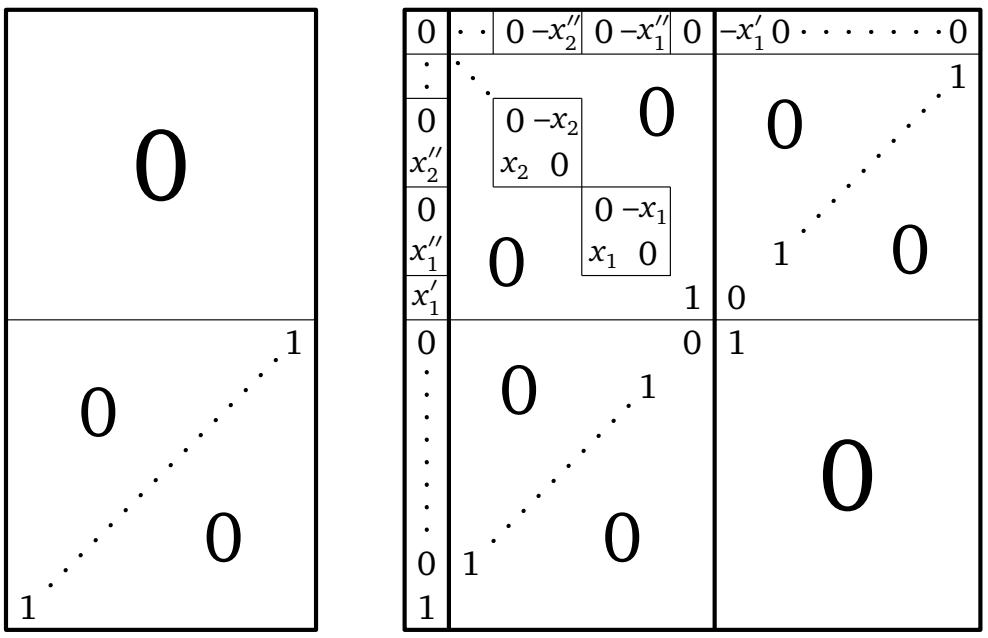

Figure 9.8

\begin{tabular}{|c|l|l|r|}
\hline & \multicolumn{1}{|c|}{$\operatorname{deg}$} & \multicolumn{1}{|c|}{ Weight } & \multicolumn{1}{|c|}{ Geometric condition } \\
\hline$F_{k}^{2}$ & $(1,0,1)_{P l}$ & $2 \pi_{l-1-2 k}$ & $\left\langle R_{l} \cap E_{l+1+2 k}+S_{l}^{\prime} \cap E_{l+1+2 k}\right\rangle \cap E_{l-1-2 k} \neq 0$, \\
$F_{k}$ & $(1,0,1)$ & $\omega_{l-1-2 k}$ & $k=1, \ldots,[(l-1) / 2]$ \\
\hline$F_{1}^{\prime 2}$ & $(1,2,0)_{P l}$ & $\pi_{l}^{\prime}$ & $\left\langle R_{l}+S_{1}\right\rangle \cap E_{l}^{\prime} \perp S_{1}$ \\
$F_{1}^{\prime}$ & $(1,1,0)$ & $\omega_{l-1}$ & \\
\hline$F_{k}^{\prime \prime 2}$ & $(1,2,1)_{P l}$ & $2 \pi_{l-2 k}$ & $\left\langle\left\langle R_{l}+S_{1}\right\rangle \cap E_{l+2 k}+S_{l}^{\prime} \cap E_{l+2 k}\right\rangle \cap E_{l-2 k} \perp S_{1}$, \\
$F_{k}^{\prime \prime}$ & $(1,1,1)$ & $\omega_{l-2 k}$ & $k=1, \ldots,[(l-2) / 2]$ \\
\hline
\end{tabular}


9. $(p, p),(1,1, q, 1,1), p=l$.

We can assume that $p \geq 4$. (The case of $p=3$ can be omitted for the same reason as in case 4 on p. 109.)
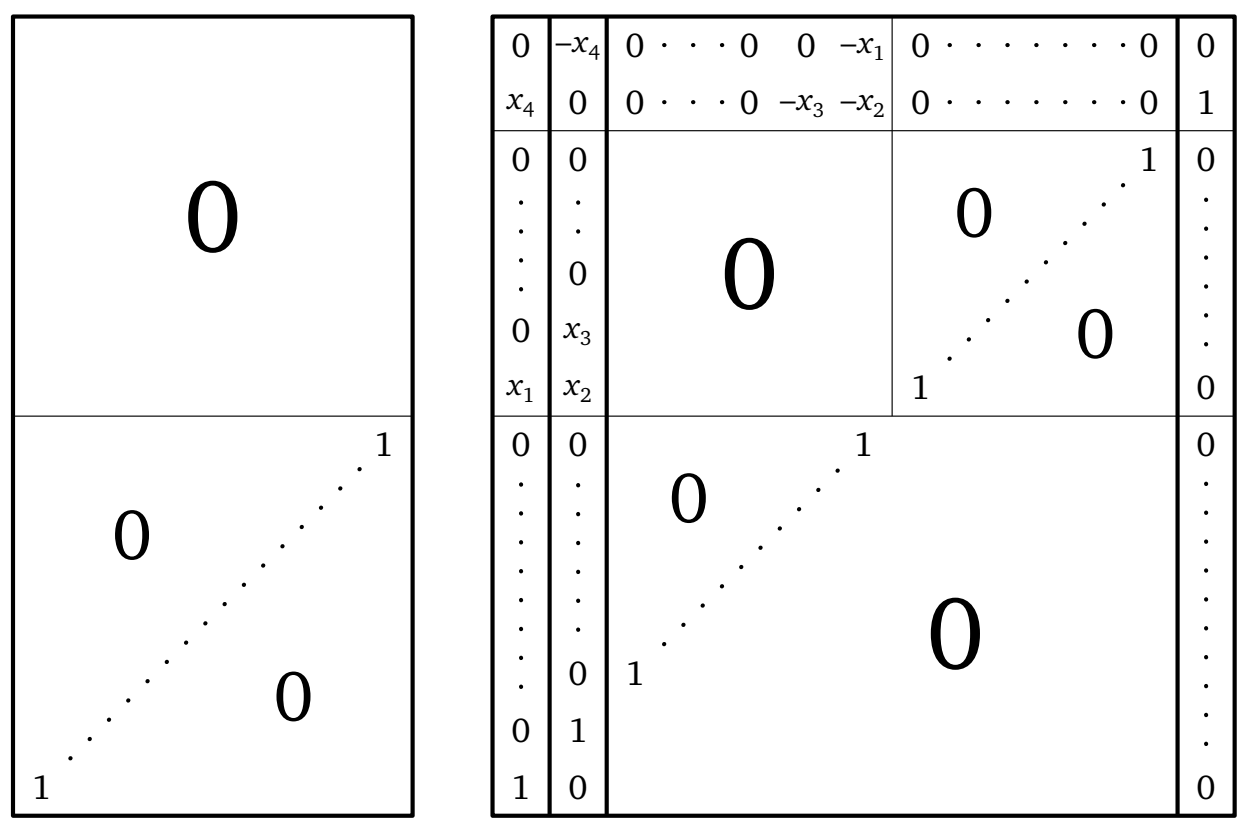

FIGURE 9.9

\begin{tabular}{|l|l|l|c|}
\hline & \multicolumn{1}{|c|}{$\operatorname{deg}$} & Weight & \multicolumn{1}{|c|}{ Geometric condition } \\
\hline$F_{1}^{2}$ & $(1,2,0)_{P l}$ & $\pi_{l}^{\prime}$ & $\left\langle R_{l}+S_{1}\right\rangle \cap E_{l}^{\prime} \perp S_{1}$ \\
$F_{1}$ & $(1,1,0)$ & $\omega_{l-1}$ & \\
\hline$F_{2}^{2}$ & $(1,0,2)_{P l}$ & $2 \pi_{1}+\pi_{l}^{\prime}$ & $\left\langle R_{l} \cap E_{n-1}+S_{2} \cap E_{n-1}\right\rangle \cap E_{l}^{\prime} \perp S_{2} \cap E_{n-1}$ \\
$F_{2}$ & $(1,0,1)$ & $\omega_{1}+\omega_{l-1}$ & \\
\hline$F_{3}$ & $(1,0,1)_{P l}$ & $\pi_{l-2}$ & $\left\langle R_{l}+S_{2}\right\rangle \cap E_{l-2} \neq 0$ \\
$F_{3}$ & $(2,0,1)$ & $\omega_{l-2}$ & \\
\hline$F_{4}^{2}$ & $(1,0,2)_{P l}$ & $\pi_{l}$ & $\left\langle\left\langle R_{l}+S_{2}\right\rangle \cap E_{l}+S_{n-2} \cap E_{l}\right\rangle \cap E_{0} \neq 0$ \\
$F_{4}$ & $(1,0,1)$ & $\omega_{l}$ & \\
\hline
\end{tabular}

10. $(p, 1, p),(p, 1, p), p=l$.

\begin{tabular}{|c|l|l|c|}
\hline & \multicolumn{1}{|c|}{$\operatorname{deg}$} & Weight & Geometric condition \\
\hline$F_{k}^{2}$ & $(1,1)_{P l}$ & $2 \pi_{l-k}$ & $\left\langle R_{l+1} \cap E_{l+k+1}+S_{l} \cap E_{l+k+1}\right\rangle \cap E_{l-k} \neq 0, k=1, \ldots, l$ \\
$F_{k}$ & $(1,1)$ & $\omega_{l-k}$ & \\
\hline
\end{tabular}



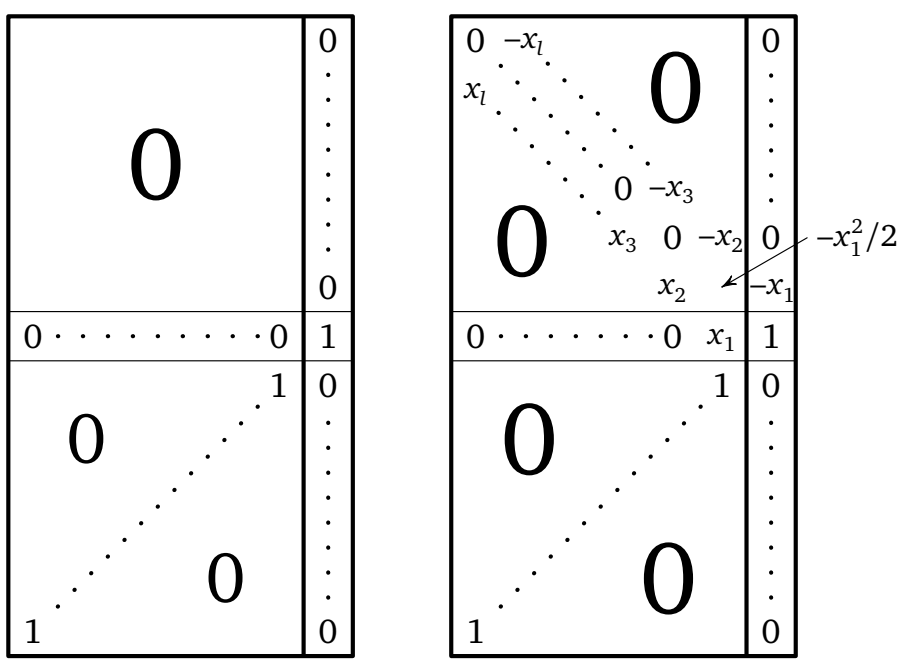

FiguRE 9.10

11. $(1, p, 1),\left(q_{1}, q_{2}, q_{1}\right)$. The cases in which $q_{1}=1$ and $q_{2}=1,2$ can be omitted, because $\mathrm{SO}_{3}$ and $\mathrm{SO}_{4}$ are locally isomorphic to $\mathrm{SL}_{2}$ and $\mathrm{SL}_{2} \times \mathrm{SL}_{2}$, respectively.

(a) $q_{1}>1, q_{2}>2$.
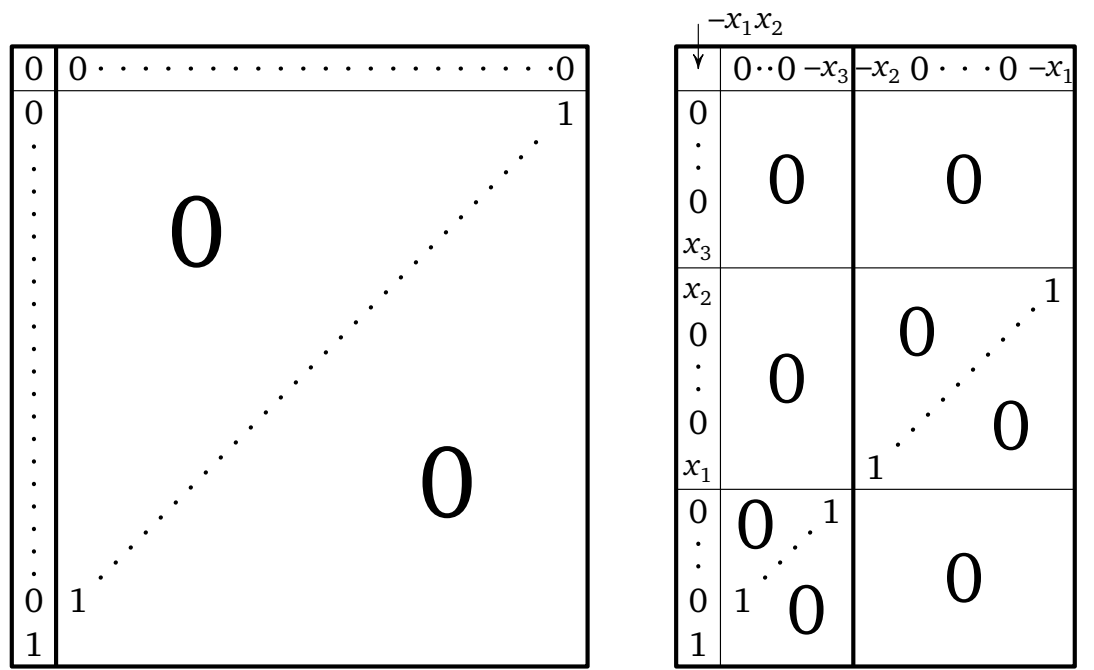

FiguRE $9.11 \mathrm{a}$

\begin{tabular}{|c|c|l|c|}
\hline & deg & Weight & Geometric condition \\
\hline$F_{1}$ & $(1,1)$ & $\pi_{q_{1}+1}$ & $\left\langle R_{1}+S_{q_{1}}\right\rangle \cap E_{n-q_{1}-1} \neq 0$ \\
\hline$F_{2}$ & $(2,1)$ & $\omega_{q_{1}}$ & $\left\langle R_{1}+S_{q_{1}}\right\rangle \cap E_{n-q_{1}} \perp R_{1}$ \\
\hline$F_{3}$ & $(1,1)$ & $\omega_{q_{1}-1}$ & $\left\langle R_{1}+S_{n-q_{1}}\right\rangle \cap E_{q_{1}-1} \neq 0$ \\
\hline
\end{tabular}

(b) $q_{1}=1$ and $q_{2}>2$. In this case, $f_{2}=F_{3} F_{P, 1}$. Hence div $f_{2}$ is not simple, and one should delete $F_{2}$ from the generators. 
(c) $q_{1}>1$ and $q_{2}=1\left(q_{1}=l\right)$.
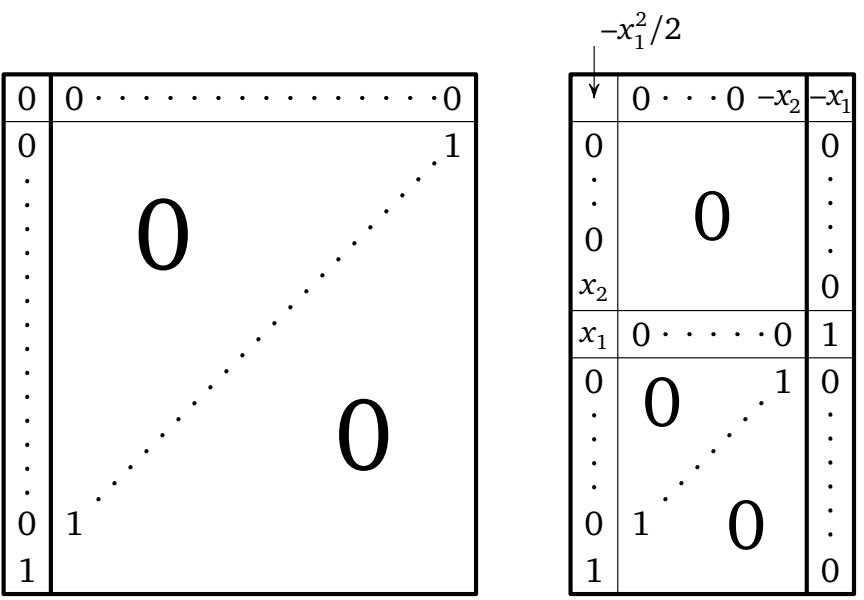

FiguRE $9.11 b$

\begin{tabular}{|c|l|l|c|}
\hline & \multicolumn{1}{|c|}{$\operatorname{deg}$} & Weight & Geometric condition \\
\hline$F_{1}^{2}$ & $(2,1)_{P l}$ & $\pi_{l}$ & $\left\langle R_{1}+S_{l}\right\rangle \cap E_{l+1} \perp R_{1}$ \\
$F_{1}$ & $(1,1)$ & $\omega_{l}$ & \\
\hline$F_{3}$ & $(1,1)_{P l}$ & $\pi_{l-1}$ & $\left\langle R_{1}+S_{l+1}\right\rangle \cap E_{l-1} \neq 0$ \\
$F_{3}$ & $(1,2)$ & $\omega_{l-1}$ & \\
\hline
\end{tabular}

(d) $q_{1}>1$ and $q_{2}=2$. This case coincides with case $12 \mathrm{c}$.

12. $(1, p, 1),\left(q_{1}, q_{2}, q_{2}, q_{1}\right)$. Note that the case in which $q_{1}=1$ and $q_{2}=1$ can be omitted. (a) $q_{1}, q_{2}>1$.

\begin{tabular}{|l|l|l|c|}
\hline & \multicolumn{1}{|c|}{$\operatorname{deg}$} & Weight & \multicolumn{1}{c|}{ Geometric condition } \\
\hline$F_{1}$ & $(1,1,0)_{P l}$ & $\pi_{q_{1}+1}$ & $\left\langle R_{1}+S_{q_{1}}\right\rangle \cap E_{n-q_{1}-1} \neq 0$ \\
$F_{1}$ & $(1,1,0)$ & $\pi_{q_{1}+1}$ & \\
\hline$F_{2}^{2}$ & $(2,0,1)_{P l}$ & $\pi_{l}^{\prime}$ & $\left\langle R_{1}+S_{l}\right\rangle \cap E_{l}^{\prime} \perp R_{1}$ \\
$F_{2}$ & $(1,0,1)$ & $\omega_{l-1}$ & \\
\hline$F_{3}$ & $(1,1,0)_{P l}$ & $\pi_{q_{1}-1}$ & $\left\langle R_{1}+S_{n-q_{1}}\right\rangle \cap E_{q_{1}-1} \neq 0$ \\
$F_{3}$ & $(1,1,0)$ & $\omega_{q_{1}-1}$ & \\
\hline$F_{4}$ & $(2,1,0)_{P l}$ & $\pi_{q_{1}}$ & $\left\langle R_{1}+S_{q_{1}}\right\rangle \cap E_{n-q_{1}} \perp R_{1}$ \\
$F_{4}$ & $(2,1,0)$ & $\omega_{q_{1}}$ & \\
\hline
\end{tabular}



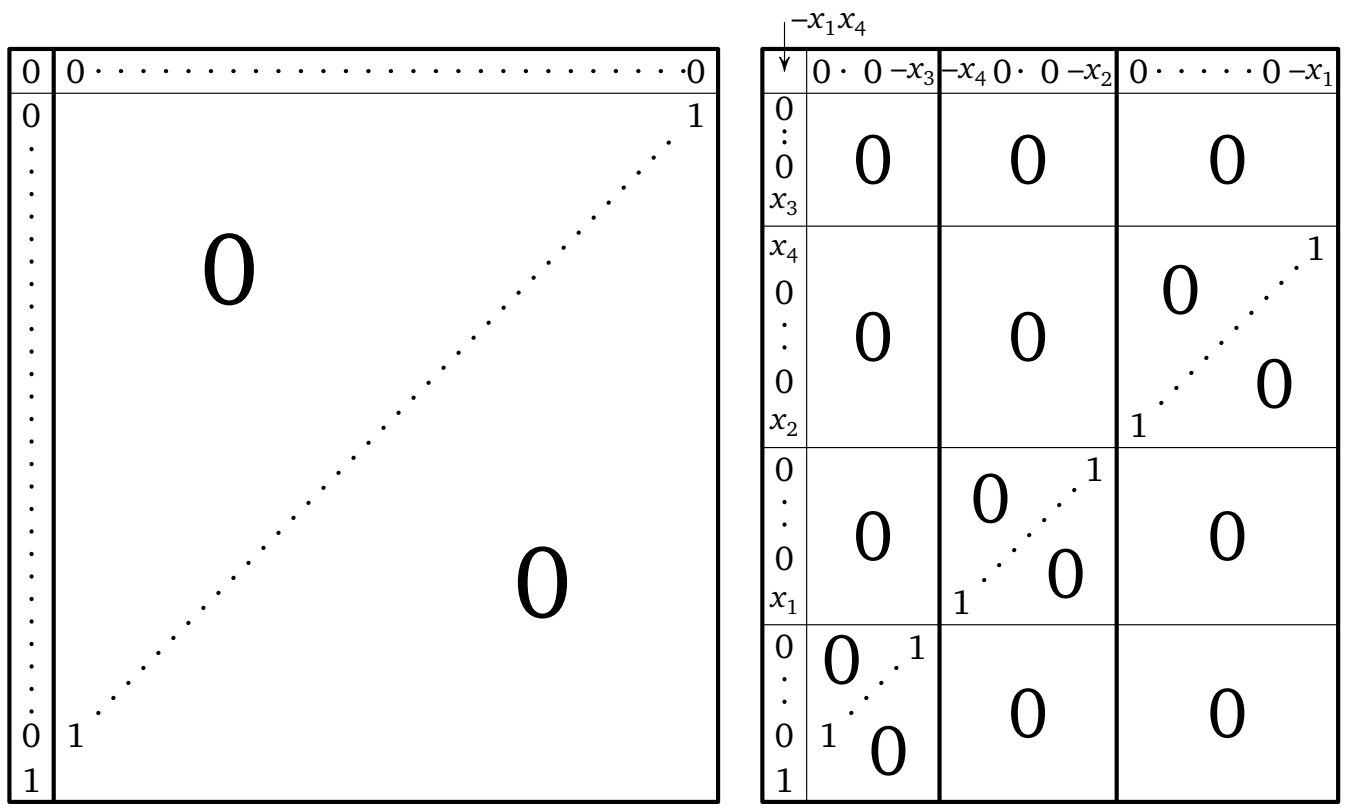

FiguRE 9.12a

(b) $q_{1}=1$ and $q_{2}>1$. In this case, $f_{4}=F_{3} F_{P, 1}$. Hence $\operatorname{div} f_{4}$ is not simple, and one should delete $F_{4}$ from the generators.

(c) $q_{1}>1$ and $q_{2}=1$.
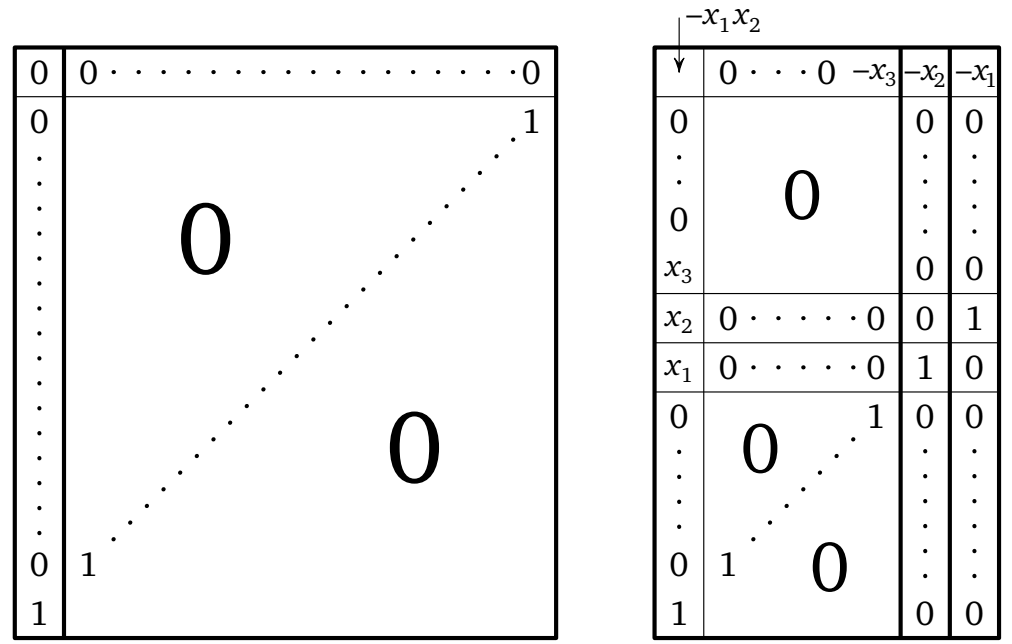

FiguRe 9.12.b 


\begin{tabular}{|c|l|l|c|}
\hline & \multicolumn{1}{|c|}{$\operatorname{deg}$} & Weight & \multicolumn{1}{c|}{ Geometric condition } \\
\hline$F_{1}^{2}$ & $(2,0,0,1)_{P l}$ & $\pi_{l}$ & $\left\langle R_{1}+S_{l}^{\prime}\right\rangle \cap E_{l} \perp R_{1}$ \\
$F_{1}$ & $(1,1,0)$ & $\omega_{l}$ & \\
\hline$F_{2}^{2}$ & $(2,0,1,0)_{P l}$ & $\pi_{l}^{\prime}$ & $\left\langle R_{1}+S_{l}\right\rangle \cap E_{l}^{\prime} \perp R_{1}$ \\
$F_{2}$ & $(1,0,1)$ & $\omega_{l-1}$ & \\
\hline$F_{3}$ & $(1,1,0,0)_{P l}$ & $\pi_{l-2}$ & $\left\langle R_{1}+S_{n-q_{1}}\right\rangle \cap E_{q_{1}-1} \neq 0$ \\
$F_{3}$ & $(1,1,1)$ & $\omega_{l-2}$ & \\
\hline
\end{tabular}

13. $(4,4),(2,2,2,2)^{\prime}$.

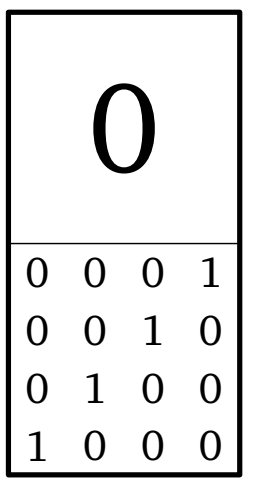

\begin{tabular}{|cc|cc|cc|}
\hline 0 & $-x_{4}$ & $-x_{2}$ & 0 & 0 & 0 \\
$x_{4}$ & 0 & $-x_{3}$ & 0 & $-x_{1}$ & 0 \\
$x_{2}$ & $x_{3}$ & 0 & 0 & 0 & 1 \\
0 & $x_{1}$ & 0 & 1 & 0 & 0 \\
\hline 0 & 0 & 0 & 0 & 1 & 0 \\
0 & 0 & 1 & 0 & 0 & 0 \\
0 & 1 & 0 & 0 & 0 & 0 \\
1 & 0 & 0 & 0 & 0 & 0 \\
\hline
\end{tabular}

Figure 9.13

\begin{tabular}{|l|l|l|l|}
\hline & \multicolumn{1}{|c|}{$\operatorname{deg}$} & Weight & \multicolumn{1}{c|}{ Geometric condition } \\
\hline$F_{1}^{2}$ & $(1,2,0)_{P l}$ & $2 \pi_{1}+\pi_{4}^{\prime}$ & $\left\langle R_{4} \cap E_{7}+S_{2} \cap E_{7}\right\rangle \cap E_{4}^{\prime} \perp S_{2} \cap E_{7}$ \\
$F_{1}$ & $(1,1,0)$ & $\omega_{1}+\omega_{3}$ & \\
\hline$F_{2}$ & $(1,1,0)_{P l}$ & $\pi_{2}$ & $\left\langle R_{4}+S_{2}\right\rangle \cap E_{2} \neq 0$ \\
$F_{2}$ & $(2,1,0)$ & $\omega_{2}$ & \\
\hline$F_{3}^{2}$ & $(1,0,1)_{P l}$ & $2 \pi_{1}$ & $\left\langle R_{4} \cap E_{7}+S_{4} \cap E_{7}\right\rangle \cap E_{1} \neq 0$ \\
$F_{3}$ & $(1,0,1)$ & $\omega_{1}$ & \\
\hline$F_{4}^{2}$ & $(1,2,0)_{P l}$ & $\pi_{4}$ & $\left\langle\left\langle R_{4}+S_{2}\right\rangle \cap E_{4}+S_{6} \cap E_{4}\right\rangle \cap E_{0} \neq 0$ \\
$F_{4}$ & $(1,1,0)$ & $\omega_{4}$ & \\
\hline
\end{tabular}




\subsection{Complexity 1.}

14. $(2, p, 2),(q, 1, q), q=l$.

(a) $p \geq 5$.
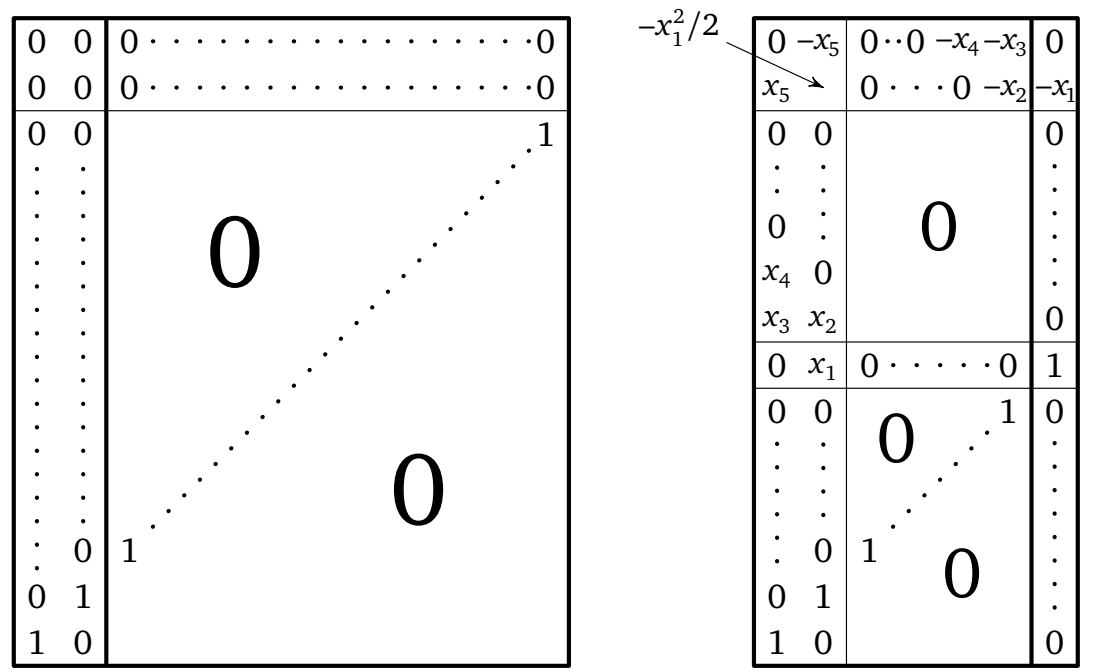

FiguRE 9.14

\begin{tabular}{|c|l|l|c|}
\hline & \multicolumn{1}{|c|}{$\operatorname{deg}$} & \multicolumn{1}{|c|}{ Weight } & \multicolumn{1}{c|}{ Geometric condition } \\
\hline$F_{1}^{2}$ & $(2,1)_{P l}$ & $2 \pi_{1}+\pi_{l}$ & $\left\langle R_{2} \cap E_{n-1}+S_{l} \cap E_{n-1}\right\rangle \cap E_{l+1} \perp R_{2} \cap E_{n-1}$ \\
$F_{1}$ & $(1,1)$ & $\omega_{1}+\omega_{l}$ & \\
\hline$F_{2}$ & $(1,1)_{P l}$ & $\pi_{1}+\pi_{l-1}$ & $\left\langle R_{2} \cap E_{n-1}+S_{l+1} \cap E_{n-1}\right\rangle \cap E_{l-1} \neq 0$ \\
$F_{2}$ & $(1,2)$ & $\omega_{1}+\omega_{l-1}$ & \\
\hline$F_{3}$ & $(1,1)_{P l}$ & $\pi_{l-1}$ & $\left\langle R_{2}+S_{l}\right\rangle \cap E_{l-1} \neq 0$ \\
$F_{3}$ & $(1,2)$ & $\omega_{l-1}$ & $\left\langle R_{2}+S_{l+1}\right\rangle \cap E_{l-2} \neq 0$ \\
\hline$F_{4}$ & $(1,1)_{P l}$ & $\pi_{l-2}$ & \\
$F_{4}$ & $(1,2)$ & $\omega_{l-2}$ & $\left\langle\left\langle R_{2}+S_{l}\right\rangle \cap E_{l+1}+R_{n-2} \cap E_{l+1}\right\rangle \cap E_{0} \neq 0$ \\
\hline$F_{5}^{2}$ & $(2,1)_{P l}$ & $\pi_{l}$ & \\
$F_{5}$ & $(1,1)$ & $\omega_{l}$ & $\left\langle\left\langle R_{2} \cap E_{n-1}+S_{l} \cap E_{n-1}\right\rangle \cap E_{l+2}+R_{n-2} \cap E_{l+2}\right\rangle$ \\
\hline$F_{6}$ & $(2,1)_{P l}$ & $\pi_{1}+\pi_{l-1}$ & \\
$F_{6}$ & $(2,2)$ & $\omega_{1}+\omega_{l-1}$ & \\
\hline$F_{1} F_{3}$ & & & \\
$F_{2} F_{5}$ & $(2,3)$ & $\omega_{1}+\omega_{l-1}+\omega_{l}$ & \\
$F_{6} F_{Q, l}$ & & & \\
\hline
\end{tabular}

(b) $p=3$. If $p=3$, then $f_{6}=F_{4} F_{P, 2}$. Hence $\operatorname{div} f_{6}$ is not simple, and one should delete $F_{6}$ from the generators and replace $F_{6}$ by $F_{4} F_{P, 2}$ in the relation. 
15. $(1, p, 1),\left(q_{1}, q_{2}, q_{3}, q_{2}, q_{1}\right)$.

(a) $q_{1}, q_{2}>1$ and $q_{3}>2$.
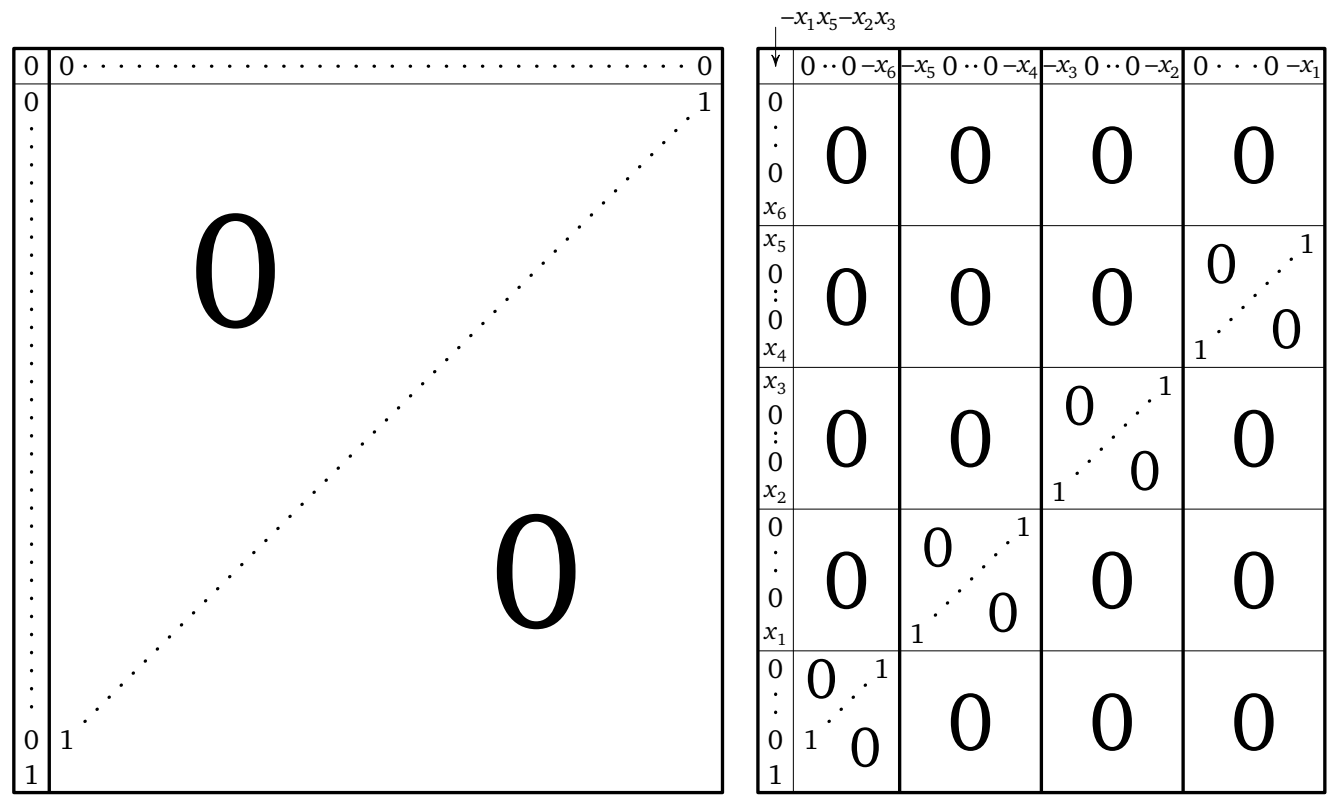

FIGURE 9.15

\begin{tabular}{|c|c|l|l|}
\hline & deg & Weight & \multicolumn{1}{c|}{ Geometric condition } \\
\hline$F_{1}$ & $(1,1,0)$ & $\omega_{q_{1}+1}$ & $\left\langle R_{1}+S_{q_{1}}\right\rangle \cap E_{n-q_{1}-1} \neq 0$ \\
\hline$F_{2}$ & $(1,0,1)$ & $\pi_{q_{1}+q_{2}+1}$ & $\left\langle R_{1}+S_{q_{1}+q_{2}}\right\rangle \cap E_{n-\left(q_{1}+q_{2}\right)-1} \neq 0$ \\
\hline$F_{3}$ & $(2,0,1)$ & $\omega_{q_{1}+q_{2}}$ & $\left\langle R_{1}+S_{q_{1}+q_{2}}\right\rangle \cap E_{n-\left(q_{1}+q_{2}\right)} \perp R_{1}$ \\
\hline$F_{4}$ & $(1,0,1)$ & $\omega_{q_{1}+q_{2}-1}$ & $\left\langle R_{1}+S_{n-\left(q_{1}+q_{2}\right)}\right\rangle \cap E_{q_{1}+q_{2}-1} \neq 0$ \\
\hline$F_{6}$ & $(1,1,0)$ & $\omega_{q_{1}-1}$ & $\left\langle R_{1}+S_{n-q_{1}}\right\rangle \cap E_{q_{1}-1} \neq 0$ \\
\hline$F_{7}$ & $(2,1,0)$ & $\omega_{q_{1}}$ & $\left\langle R_{1}+S_{q_{1}}\right\rangle \cap E_{n-q_{1}} \perp R_{1}$ \\
\hline$F_{3} F_{Q, q_{1}}$ & $(2,1,1)$ & $\omega_{q_{1}}+\omega_{q_{1}+q_{2}}$ & \\
$F_{7} F_{Q, q_{1}+q_{2}}$ & & & \\
\hline
\end{tabular}

The divisor $D_{5}$ given by the condition

$$
\left\langle R_{1}+S_{q_{1}}\right\rangle \cap E_{n-q_{1}} \perp\left\langle R_{1}+S_{n-\left(q_{1}+q_{2}\right)}\right\rangle \cap E_{q_{1}+q_{2}}
$$

is parametric.

Here $R(X)^{U}$ is free.

(b) $q_{1}=1$ or $q_{2}=1 ; q_{3}>2$.

If $q_{1}=1$, then $f_{7}=F_{6} F_{P, 1}$. Hence $\operatorname{div} f_{7}$ is not simple, and one should delete $F_{7}$ from the generators and replace $F_{7}$ by $F_{6} F_{P, 1}$ in the product. 
If $q_{2}=1$, then $f_{5}=F_{4} F_{1}$. Hence there exist three exceptional points, yet another form product $F_{4} F_{1}$ of the same weight and multidegree as $F$ and $F^{\prime}$ occurs, and $R(X)^{U}$ proves to be a hypersurface.

(c) $q_{3}=2$. This is a special case of case 16 on p. 120 .

(d) $q_{1}, q_{2}>1$ and $q_{3}=1$.

\begin{tabular}{|c|l|l|c|}
\hline & \multicolumn{1}{|c|}{ deg } & \multicolumn{1}{|c|}{ Weight } & Geometric condition \\
\hline$F_{1}$ & $(1,1,0)_{P l}$ & $\pi_{q_{1}+1}$ & $\left\langle R_{1}+S_{q_{1}}\right\rangle \cap E_{n-q_{1}-1} \neq 0$ \\
$F_{1}$ & $(1,1,0)$ & $\omega_{q_{1}+1}$ & \\
\hline$F_{2}^{2}$ & $(2,0,1)_{P l}$ & $\pi_{l}$ & $\left\langle R_{1}+S_{l}\right\rangle \cap E_{l+1} \perp R_{1}$ \\
$F_{2}$ & $(1,0,1)$ & $\omega_{l}$ & \\
\hline$F_{4}$ & $(1,0,1)_{P l}$ & $\pi_{l-1}$ & $\left\langle R_{1}+S_{l+1}\right\rangle \cap E_{l-1} \neq 0$ \\
$F_{4}$ & $(1,0,2)$ & $\omega_{l-1}$ & \\
\hline$F_{6}$ & $(1,1,0)_{P l}$ & $\pi_{q_{1}-1}$ & $\left\langle R_{1}+S_{n-q_{1}}\right\rangle \cap E_{q_{1}-1} \neq 0$ \\
$F_{6}$ & $(1,1,0)$ & $\omega_{q_{1}-1}$ & \\
\hline$F_{7}$ & $(2,1,0)_{P l}$ & $\pi_{q_{1}}$ & $\left\langle R_{1}+S_{q_{1}}\right\rangle \cap E_{n-q_{1}} \perp R_{1}$ \\
$F_{7}$ & $(2,1,0)$ & $\omega_{q_{1}}$ & \\
\hline$F_{2}^{2} F_{Q, q_{1}}$ & $(2,1,2)$ & $\omega_{q_{1}}+2 \omega_{l}$ & \\
$F_{7} F_{Q, l}^{2}$ & & & \\
\hline
\end{tabular}

The divisor $D_{5}$ given by the condition $\left\langle R_{1}+S_{q_{1}}\right\rangle \cap E_{n-q_{1}} \perp\left\langle R_{1}+S_{l+1}\right\rangle \cap E_{l}$ is parametric.

Here $R(X)^{U}$ is free.

(e) $q_{1}=1$ or $q_{2}=1 ; q_{3}=1$.

If $q_{1}=1$, then $f_{7}=F_{6} F_{P, 1}$. Hence $\operatorname{div} f_{7}$ is not simple, and one should delete $F_{7}$ from the generators and replace $F_{7}$ by $F_{6} F_{P, 1}$ in the product.

If $q_{2}=1$, then $f_{5}=F_{4} F_{1}$. Hence there exist three exceptional points, yet another form product $F_{4} F_{1}$ of the same weight and multidegree as $F$ and $F^{\prime}$ occurs, and $R(X)^{U}$ proves to be a hypersurface.

16. $(1, p, 1),\left(q_{1}, q_{2}, q_{3}, q_{3}, q_{2}, q_{1}\right)$.

(a) $q_{1}, q_{2}, q_{3}>1$.
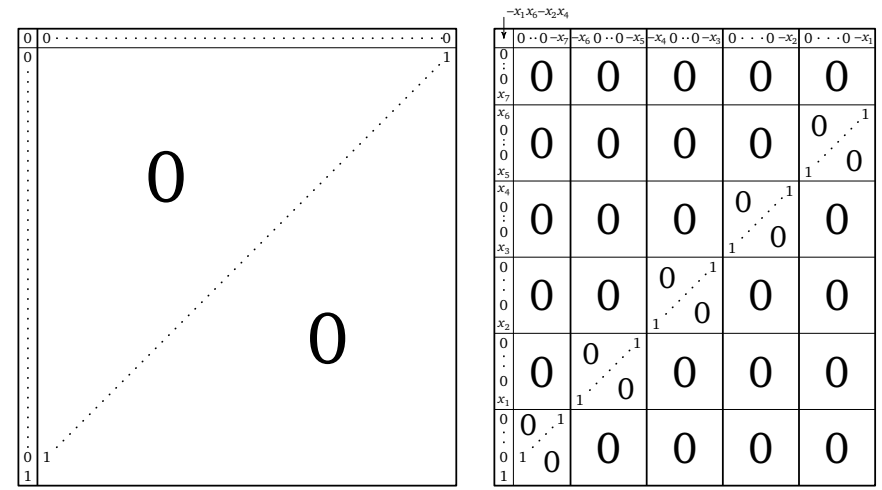

Figure 9.16 


\begin{tabular}{|c|l|l|l|}
\hline & \multicolumn{1}{|c|}{$\operatorname{deg}$} & \multicolumn{1}{c|}{ Weight } & \multicolumn{1}{c|}{ Geometric condition } \\
\hline$F_{1}$ & $(1,1,0,0)_{P l}$ & $\pi_{q_{1}+1}$ & $\left\langle R_{1}+S_{q_{1}}\right\rangle \cap E_{n-q_{1}-1} \neq 0$ \\
$F_{1}$ & $(1,1,0,0)$ & $\omega_{q_{1}+1}$ & \\
\hline$F_{2}$ & $(1,0,1,0)_{P l}$ & $\pi_{q_{1}+q_{2}+1}$ & $\left\langle R_{1}+S_{q_{1}+q_{2}}\right\rangle \cap E_{n-\left(q_{1}+q_{2}\right)-1} \neq 0$ \\
$F_{2}$ & $(1,0,1,0)$ & $\pi_{q_{1}+q_{2}+1}$ & \\
\hline$F_{3}^{2}$ & $(2,0,0,1)_{P l}$ & $\pi_{l}^{\prime}$ & $\left\langle R_{1}+S_{l}\right\rangle \cap E_{l}^{\prime} \perp R_{1}$ \\
$F_{3}$ & $(1,0,0,1)$ & $\omega_{l-1}$ & \\
\hline$F_{4}$ & $(2,0,1,0)_{P l}$ & $\pi_{q_{1}+q_{2}}$ & $\left\langle R_{1}+S_{q_{1}+q_{2}}\right\rangle \cap E_{n-q_{1}-q_{2}} \perp R_{1}$ \\
$F_{4}$ & $(2,0,1,0)$ & $\omega_{q_{1}+q_{2}}$ & \\
$F_{5}$ & $(1,0,1,0)_{P l}$ & $\pi_{q_{1}+q_{2}-1}$ & $\left\langle R_{1}+S_{n-\left(q_{1}+q_{2}\right)}\right\rangle \cap E_{q_{1}+q_{2}-1} \neq 0$ \\
$F_{5}$ & $(1,0,1,0)$ & $\omega_{q_{1}+q_{2}-1}$ & \\
\hline$F_{7}$ & $(1,1,0,0)_{P l}$ & $\pi_{q_{1}-1}$ & $\left\langle R_{1}+S_{n-q_{1}}\right\rangle \cap E_{q_{1}-1} \neq 0$ \\
$F_{7}$ & $(1,1,0,0)$ & $\omega_{q_{1}-1}$ & \\
\hline$F_{8}$ & $(2,1,0,0)_{P l}$ & $\pi_{q_{1}}$ & $\left\langle R_{1}+S_{q_{1}}\right\rangle \cap E_{n-q_{1}} \perp R_{1}$ \\
$F_{8}$ & $(2,1,0,0)$ & $\omega_{q_{1}}$ & \\
\hline$F_{4} F_{Q, q_{1}}$ & $(2,1,1,0)$ & $\omega_{q_{1}}+\omega_{q_{1}+q_{2}}$ & \\
$F_{8} F_{Q, q_{1}+q_{2}}$ & & & \\
\hline
\end{tabular}

The divisor $D_{6}$ given by the condition

$$
\left\langle R_{1}+S_{q_{1}}\right\rangle \cap E_{n-q_{1}} \perp\left\langle R_{1}+S_{n-\left(q_{1}+q_{2}\right)}\right\rangle \cap E_{q_{1}+q_{2}}
$$

is parametric.

Here $R(X)^{U}$ is free.

(b) $q_{1}=1$ or $q_{2}=1 ; q_{3}>1$.

If $q_{1}=1$, then $f_{8}=F_{7} F_{P, 1}$. Hence $\operatorname{div} f_{8}$ is not simple, and one should delete $F_{8}$ from the generators and replace $F_{8}$ by $F_{7} F_{P, 1}$ in the product.

If $q_{2}=1$, then $f_{6}=F_{5} F_{1}$. Hence there exist three exceptional points, yet another form product $F_{5} F_{1}$ of the same weight and multidegree as $F$ and $F^{\prime}$ occurs, and $R(X)^{U}$ proves to be a hypersurface.

(c) $q_{1}, q_{2}>1$ and $q_{3}=1$.

\begin{tabular}{|l|l|l|l|}
\hline & \multicolumn{1}{|c|}{$\operatorname{deg}$} & Weight & \multicolumn{1}{c|}{ Geometric condition } \\
\hline$F_{1}$ & $(1,1,0,0,0)_{P l}$ & $\pi_{q_{1}+1}$ & $\left\langle R_{1}+S_{q_{1}}\right\rangle \cap E_{n-q_{1}-1} \neq 0$ \\
$F_{1}$ & $(1,1,0,0)$ & $\omega_{q_{1}+1}$ & \\
\hline$F_{2}^{2}$ & $(2,0,0,0,1)_{P l}$ & $\pi_{l}$ & $\left\langle R_{1}+S_{l}^{\prime}\right\rangle \cap E_{l} \perp R_{1}$ \\
$F_{2}$ & $(1,0,1,0)$ & $\omega_{l}$ & \\
\hline$F_{3}^{2}$ & $(2,0,0,1,0)_{P l}$ & $\pi_{l}^{\prime}$ & $\left\langle R_{1}+S_{l}\right\rangle \cap E_{l}^{\prime} \perp R_{1}$ \\
$F_{3}$ & $(1,0,0,1)$ & $\omega_{l-1}$ & \\
\hline$F_{5}$ & $(1,0,1,0,0)_{P l}$ & $\pi_{l-2}$ & $\left\langle R_{1}+S_{l+1}\right\rangle \cap E_{l-2} \neq 0$ \\
$F_{5}$ & $(1,0,1,1)$ & $\omega_{l-2}$ & \\
\hline
\end{tabular}




\begin{tabular}{|c|l|l|c|}
\hline & \multicolumn{1}{|c|}{$\operatorname{deg}$} & \multicolumn{1}{c|}{ Weight } & Geometric condition \\
\hline$F_{7}$ & $(1,1,0,0,0)_{P l}$ & $\pi_{q_{1}-1}$ & $\left\langle R_{1}+S_{n-q_{1}}\right\rangle \cap E_{q_{1}-1} \neq 0$ \\
$F_{7}$ & $(1,1,0,0)$ & $\omega_{q_{1}-1}$ & \\
\hline$F_{8}$ & $(2,1,0,0,0)_{P l}$ & $\pi_{q_{1}}$ & $\left\langle R_{1}+S_{q_{1}}\right\rangle \cap E_{n-q_{1}} \perp R_{1}$ \\
$F_{8}$ & $(2,1,0,0)$ & $\omega_{q_{1}}$ & \\
\hline$F_{2} F_{3} F_{Q, q_{1}}$ & $(2,1,1,1)$ & $\omega_{q_{1}}+\omega_{l-1}+\omega_{l}$ & \\
$F_{8} F_{Q, l}^{\prime} F_{Q, l}$ & & & \\
\hline
\end{tabular}

The divisor $D_{6}$ given by the condition

$$
\left\langle R_{1}+S_{q_{1}}\right\rangle \cap E_{n-q_{1}} \perp\left\langle R_{1}+S_{l+1}\right\rangle \cap E_{l-1}
$$

is parametric.

Here $R(X)^{U}$ is free.

(d) $q_{1}=1$ or $q_{2}=1 ; q_{3}=1$.

If $q_{1}=1$, then $f_{8}=F_{7} F_{P, 1}$. Hence $\operatorname{div} f_{8}$ is not simple, and one should delete $F_{8}$ from the generators and replace $F_{8}$ by $F_{7} F_{P, 1}$ in the product.

If $q_{2}=1$, then $f_{6}=F_{5} F_{1}$. Hence there exist three exceptional points, yet another form product $F_{5} F_{1}$ of the same weight and multidegree as $F$ and $F^{\prime}$ occurs, and $R(X)^{U}$ proves to be a hypersurface.

17. $(4,4),(2,2,2,2)$.

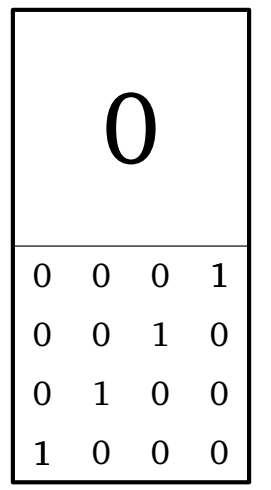

\begin{tabular}{|cc|cc|cc|}
\hline 0 & $-x_{4}$ & $-x_{3}$ & 0 & 0 & 0 \\
$x_{4}$ & 0 & 0 & $-x_{2}$ & 0 & 0 \\
$x_{3}$ & 0 & 0 & $-x_{1}$ & 0 & 1 \\
0 & $x_{2}$ & $x_{1}$ & 0 & 1 & 0 \\
\hline 0 & 0 & 0 & 1 & 0 & 0 \\
0 & 0 & 1 & 0 & 0 & 0 \\
0 & 1 & 0 & 0 & 0 & 0 \\
1 & 0 & 0 & 0 & 0 & 0 \\
\hline
\end{tabular}

FiguRE 9.17

\begin{tabular}{|c|l|l|c|}
\hline & \multicolumn{1}{|c|}{$\operatorname{deg}$} & Weight & Geometric condition \\
\hline$F_{1}^{2}$ & $(1,0,1)_{P l}$ & $2 \pi_{2}$ & $\left\langle R_{4} \cap E_{6}+S_{4} \cap E_{6}\right\rangle \cap E_{2} \neq 0$ \\
$F_{1}$ & $(1,0,1)$ & $\omega_{2}$ & \\
\hline$F_{2}^{2}$ & $(1,2,0)_{P l}$ & $2 \pi_{1}+\pi_{4}^{\prime}$ & $\left\langle R_{4} \cap E_{7}+S_{2} \cap E_{7}\right\rangle \cap E_{4}^{\prime} \perp S_{2} \cap E_{7}$ \\
$F_{2}$ & $(1,1,0)$ & $\omega_{1}+\omega_{3}$ & \\
\hline$F_{3}$ & $(1,1,0)_{P l}$ & $\pi_{2}$ & $\left\langle R_{4}+S_{2}\right\rangle \cap E_{2} \neq 0$ \\
$F_{3}$ & $(2,1,0)$ & $\omega_{2}$ & \\
\hline
\end{tabular}




\begin{tabular}{|c|l|l|c|}
\hline & \multicolumn{1}{|c|}{$\operatorname{deg}$} & Weight & Geometric condition \\
\hline$F_{4}^{2}$ & $(1,2,0)_{P l}$ & $\pi_{4}$ & $\left\langle\left\langle R_{4}+S_{2}\right\rangle \cap E_{4}+S_{6} \cap E_{4}\right\rangle \cap E_{0} \neq 0$ \\
$F_{4}$ & $(1,1,0)$ & $\omega_{4}$ & \\
\hline$F_{5}^{2}$ & $(1,0,1)_{P l}$ & 0 & $\left\langle R_{4}+S_{4}\right\rangle \cap E_{0} \neq 0$ \\
$F_{5}$ & $(1,0,1)$ & 0 & \\
\hline$F_{1} F_{4}$ & & & \\
$F_{3} F_{Q, 4}$ & $(2,1,1)$ & $\omega_{2}+\omega_{4}$ & \\
$F_{5} F_{P, 4} F_{Q, 2}$ & & & \\
\hline
\end{tabular}

18. $(4,4),(1,2,2,2,1)$.

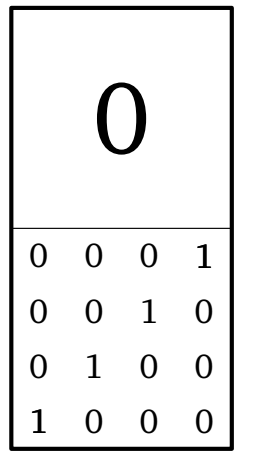

\begin{tabular}{|c|cc|cc|cc|}
\hline 0 & $-x_{5}$ & $-x_{4}$ & $-x_{3}$ & 0 & 0 & 0 \\
$x_{5}$ & 0 & $-x_{2}$ & 0 & 0 & 0 & 1 \\
$x_{4}$ & $x_{2}$ & 0 & $-x_{1}$ & 0 & 1 & 0 \\
$x_{3}$ & 0 & $x_{1}$ & 0 & 1 & 0 & 0 \\
\hline 0 & 0 & 0 & 1 & 0 & 0 & 0 \\
0 & 0 & 1 & 0 & 0 & 0 & 0 \\
0 & 1 & 0 & 0 & 0 & 0 & 0 \\
1 & 0 & 0 & 0 & 0 & 0 & 0 \\
\hline
\end{tabular}

Figure 9.18

\begin{tabular}{|c|l|l|l|}
\hline & \multicolumn{1}{|c|}{ deg } & \multicolumn{1}{|c|}{ Weight } & \multicolumn{1}{c|}{ Geometric condition } \\
\hline$F_{1}^{2}$ & $(1,0,0,1,0)_{P l}$ & $2 \pi_{2}$ & $\left\langle R_{4} \cap E_{6}+S_{4} \cap E_{6}\right\rangle \cap E_{2} \neq 0$ \\
$F_{1}$ & $(1,0,0,1)$ & $\omega_{2}$ & \\
\hline$F_{2}^{2}$ & $(1,0,0,0,1)_{P l}$ & $2 \pi_{1}$ & $\left\langle R_{4} \cap E_{7}+S_{4}^{\prime} \cap E_{7}\right\rangle \cap E_{1} \neq 0$ \\
\hline$F_{2}$ & $(1,0,1,0)$ & $\omega_{1}$ & \\
\hline$F_{3}^{2}$ & $(1,2,0,0,0)_{P l}$ & $\pi_{4}^{\prime}$ & $\left\langle R_{4}+S_{1}\right\rangle \cap E_{4}^{\prime} \perp S_{1}$ \\
$F_{3}$ & $(1,1,0,0)$ & $\omega_{3}$ & $\left\langle\left\langle R_{4}+S_{1}\right\rangle \cap E_{6}+S_{4}^{\prime} \cap E_{6}\right\rangle \cap E_{2} \perp S_{1}$ \\
\hline$F_{4}^{2}$ & $(1,2,0,0,1)_{P l}$ & $\pi_{2}$ & \\
$F_{4}$ & $(1,1,1,0)$ & $\omega_{2}$ & $\left\langle R_{4}+S_{4}\right\rangle \cap E_{0} \neq 0$ \\
\hline$F_{6}^{2}$ & $(1,0,0,1,0)_{P l}$ & 0 & \\
\hline$F_{6}$ & $(1,0,0,1)$ & 0 & \\
\hline$F_{2} F_{3} F_{Q, 4}$ & $(2,1,1,1)$ & $\omega_{1}+\omega_{3}+\omega_{4}$ & \\
$F_{6} F_{P, 4} F_{Q, 1} F_{Q, 4}^{\prime}$ & & & \\
\hline
\end{tabular}

The divisor $D_{5}$ given by the condition $\left\langle R_{4} \cap E_{7}+S_{3} \cap E_{7}\right\rangle \cap E_{3} \perp S_{1}$ is parametric. 
19. $(4,4),(2,1,2,1,2)$.

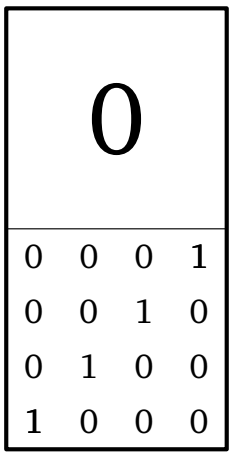

\begin{tabular}{|cc|c|cc|c|}
\hline 0 & $-x_{5}$ & $-x_{3}$ & 0 & 0 & 0 \\
$x_{5}$ & 0 & $-x_{4}$ & $-x_{2}$ & 0 & 0 \\
$x_{3}$ & $x_{4}$ & 0 & $-x_{1}$ & 0 & 1 \\
0 & $x_{2}$ & $x_{1}$ & 0 & 1 & 0 \\
\hline 0 & 0 & 0 & 1 & 0 & 0 \\
0 & 0 & 1 & 0 & 0 & 0 \\
0 & 1 & 0 & 0 & 0 & 0 \\
1 & 0 & 0 & 0 & 0 & 0 \\
\hline
\end{tabular}

FiguRE 9.19

\begin{tabular}{|c|l|l|c|}
\hline & \multicolumn{1}{|c|}{ deg } & Weight & \multicolumn{1}{c|}{ Geometric condition } \\
\hline$F_{1}^{2}$ & $(1,0,0,1,0)_{P l}$ & $2 \pi_{2}$ & $\left\langle R_{4} \cap E_{6}+S_{4} \cap E_{6}\right\rangle \cap E_{2} \neq 0$ \\
$F_{1}$ & $(1,0,0,1)$ & $\omega_{2}$ & \\
\hline$F_{2}^{2}$ & $(1,2,0,0,0)_{P l}$ & $2 \pi_{1}+\pi_{4}^{\prime}$ & $\left\langle R_{4} \cap E_{7}+S_{2} \cap E_{7}\right\rangle \cap E_{4}^{\prime} \perp S_{2} \cap E_{7}$ \\
$F_{2}$ & $(1,1,0,0)$ & $\omega_{1}+\omega_{3}$ & \\
\hline$F_{3}$ & $(1,1,0,0,0)_{P l}$ & $\pi_{2}$ & $\left\langle R_{4}+S_{2}\right\rangle \cap E_{2} \neq 0$ \\
$F_{3}$ & $(2,1,0,0)$ & $\omega_{2}$ & \\
\hline$F_{4}^{2}$ & $(1,0,0,0,1)_{P l}$ & $2 \pi_{1}$ & $\left\langle R_{4} \cap E_{7}+S_{4}^{\prime} \cap E_{7}\right\rangle \cap E_{1} \neq 0$ \\
$F_{4}$ & $(1,0,1,0)$ & $\omega_{1}$ & \\
\hline$F_{5}^{2}$ & $(1,2,0,0,0)_{P l}$ & $\pi_{4}$ & $\left\langle\left\langle R_{4}+S_{2}\right\rangle \cap E_{4}+S_{6} \cap E_{4}\right\rangle \cap E_{0} \neq 0$ \\
$F_{5}$ & $(1,1,0,0)$ & $\omega_{4}$ & \\
\hline$F_{6}^{2}$ & $(1,0,0,1,0)_{P l}$ & 0 & $\left\langle R_{4}+S_{4}\right\rangle \cap E_{0} \neq 0$ \\
$F_{6}$ & $(1,0,0,1)$ & 0 & \\
\hline$F_{1} F_{5}$ & & & \\
$F_{3} F_{Q, 4}$ & $(2,1,0,1)$ & $\omega_{2}+\omega_{4}$ & \\
$F_{6} F_{P, 4} F_{Q, 2}$ & & & \\
\hline
\end{tabular}


20. $(4,4),(1,1,2,2,1,1)$.

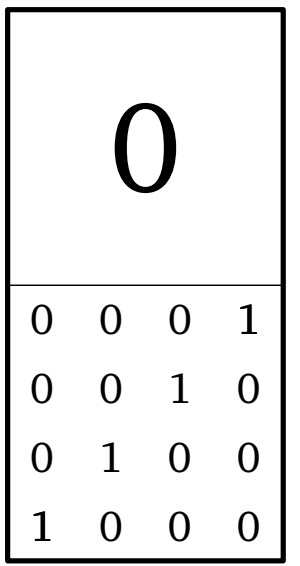

\begin{tabular}{|c|c|cc|cc|c|}
\hline 0 & $-x_{5}$ & $-x_{4}$ & $-x_{3}$ & 0 & 0 & 0 \\
$x_{5}$ & 0 & 0 & $-x_{2}$ & 0 & 0 & 1 \\
$x_{4}$ & 0 & 0 & $-x_{1}$ & 0 & 1 & 0 \\
$x_{3}$ & $x_{2}$ & $x_{1}$ & 0 & 1 & 0 & 0 \\
\hline 0 & 0 & 0 & 1 & 0 & 0 & 0 \\
0 & 0 & 1 & 0 & 0 & 0 & 0 \\
0 & 1 & 0 & 0 & 0 & 0 & 0 \\
1 & 0 & 0 & 0 & 0 & 0 & 0 \\
\hline
\end{tabular}

FiguRe 9.20

\begin{tabular}{|c|l|l|c|}
\hline & \multicolumn{1}{|c|}{ deg } & Weight & Geometric condition \\
\hline$F_{1}^{2}$ & $(1,0,0,1)_{P l}$ & $2 \pi_{2}$ & $\left\langle R_{4} \cap E_{6}+S_{4} \cap E_{6}\right\rangle \cap E_{2} \neq 0$ \\
$F_{1}$ & $(1,0,0,1)$ & $\omega_{2}$ & \\
\hline$F_{2}^{2}$ & $(1,0,2,0)_{P l}$ & $2 \pi_{1}+\pi_{4}^{\prime}$ & $\left\langle R_{4} \cap E_{7}+S_{2} \cap E_{7}\right\rangle \cap E_{4}^{\prime} \perp S_{2} \cap E_{7}$ \\
$F_{2}$ & $(1,0,1,0)$ & $\omega_{1}+\omega_{3}$ & \\
\hline$F_{3}^{2}$ & $(1,2,0,0)_{P l}$ & $\pi_{4}^{\prime}$ & $\left\langle R_{4}+S_{1}\right\rangle \cap E_{4}^{\prime} \perp S_{1}$ \\
$F_{3}$ & $(1,1,0,0)$ & $\omega_{3}$ & \\
\hline$F_{4}$ & $(1,0,1,0)_{P l}$ & $\pi_{2}$ & $\left\langle R_{4}+S_{2}\right\rangle \cap E_{2} \neq 0$ \\
$F_{4}$ & $(2,0,1,0)$ & $\omega_{2}$ & \\
\hline$F_{5}^{2}$ & $(1,0,2,0)_{P l}$ & $\pi_{4}$ & $\left\langle\left\langle R_{4}+S_{2}\right\rangle \cap E_{4}+S_{6} \cap E_{4}\right\rangle \cap E_{0} \neq 0$ \\
$F_{5}$ & $(1,0,1,0)$ & $\omega_{4}$ & \\
\hline$F_{6}^{2}$ & $(1,0,0,1)_{P l}$ & 0 & $\left\langle R_{4}+S_{4}\right\rangle \cap E_{0} \neq 0$ \\
$F_{6}$ & $(1,0,0,1)$ & 0 & \\
\hline$F_{1} F_{5}$ & & & \\
$F_{4} F_{Q, 4}$ & $(2,0,1,1)$ & $\omega_{2}+\omega_{4}$ & \\
$F_{6} F_{P, 4} F_{Q, 2}$ & & & \\
\hline
\end{tabular}


21. $(4,4),(1,1,2,2,1,1)^{\prime}$.

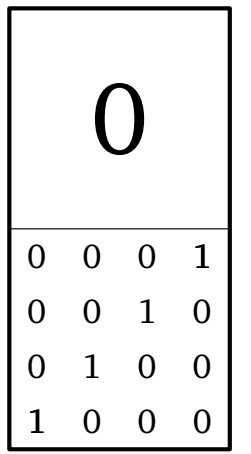

\begin{tabular}{|c|c|cc|cc|c|}
\hline 0 & $-x_{5}$ & $-x_{4}$ & 0 & $-x_{2}$ & 0 & 0 \\
$x_{5}$ & 0 & $-x_{3}$ & 0 & $-x_{1}$ & 0 & 1 \\
$x_{4}$ & $x_{3}$ & 0 & 0 & 0 & 1 & 0 \\
$x_{2}$ & $x_{1}$ & 0 & 1 & 0 & 0 & 0 \\
\hline 0 & 0 & 0 & 0 & 1 & 0 & 0 \\
0 & 0 & 1 & 0 & 0 & 0 & 0 \\
0 & 1 & 0 & 0 & 0 & 0 & 0 \\
1 & 0 & 0 & 0 & 0 & 0 & 0 \\
\hline
\end{tabular}

Figure 9.21

\begin{tabular}{|c|l|l|c|}
\hline & \multicolumn{1}{|c|}{ deg } & \multicolumn{1}{|c|}{ Weight } & \multicolumn{1}{c|}{ Geometric condition } \\
\hline$F_{1}^{2}$ & $(1,0,2,0)_{P l}$ & $2 \pi_{1}+\pi_{4}^{\prime}$ & $\left\langle R_{4} \cap E_{7}+S_{2} \cap E_{7}\right\rangle \cap E_{4}^{\prime} \perp S_{2} \cap E_{7}$ \\
$F_{1}$ & $(1,0,1,0)$ & $\omega_{1}+\omega_{3}$ & \\
\hline$F_{2}^{2}$ & $(1,2,0,0)_{P l}$ & $\pi_{4}^{\prime}$ & $\left\langle R_{4}+S_{1}\right\rangle \cap E_{4}^{\prime} \perp S_{1}$ \\
$F_{2}$ & $(1,1,0,0)$ & $\omega_{3}$ & \\
\hline$F_{3}^{2}$ & $(1,0,0,1)_{P l}$ & $2 \pi_{1}$ & $\left\langle R_{4} \cap E_{7}+S_{4}^{\prime} \cap E_{7}\right\rangle \cap E_{1} \neq 0$ \\
$F_{3}$ & $(1,0,0,1)$ & $\omega_{1}$ & \\
\hline$F_{4}^{2}$ & $(1,2,0,1)_{P l}$ & $2 \pi_{2}$ & $\left\langle\left\langle\left\langle R_{4}+S_{1}\right\rangle \cap E_{6}+S_{4}^{\prime} \cap E_{6}\right\rangle \cap E_{2}+S_{7} \cap E_{2}\right\rangle$ \\
$F_{4}$ & $(1,1,0,1)$ & $\omega_{2}$ & \\
\hline$F_{5}^{2}$ & $(1,0,2,0)_{P l}$ & $\pi_{4}$ & $\left\langle\left\langle R_{4}+S_{2}\right\rangle \cap E_{4}+S_{6} \cap E_{4}\right\rangle \cap E_{0} \neq 0$ \\
$F_{5}$ & $(1,0,1,0)$ & $\omega_{4}$ & \\
\hline$F_{6}$ & $(1,0,1,0)_{P l}$ & $\pi_{2}$ & $\left\langle R_{4}+S_{2}\right\rangle \cap E_{2} \neq 0$ \\
$F_{6}$ & $(2,0,1,0)$ & $\omega_{2}$ & \\
\hline$F_{1} F_{4}$ & & & \\
$F_{2} F_{3} F_{Q, 2}$ & $(2,1,1,1)$ & $\omega_{1}+\omega_{2}+\omega_{3}$ & \\
$F_{6} F_{Q, 1} F_{Q, 4}^{\prime}$ & & & \\
\hline
\end{tabular}


22. $(5,5),(2,3,3,2)$.

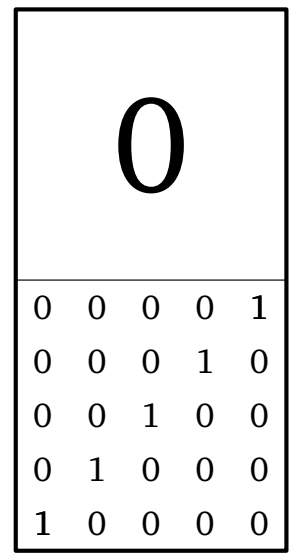

\begin{tabular}{|cc|ccc|ccc|}
\hline 0 & $-x_{6}$ & $-x_{5}$ & $-x_{3}$ & 0 & 0 & 0 & 0 \\
$x_{6}$ & 0 & $-x_{4}$ & 0 & $-x_{2}$ & 0 & 0 & 0 \\
$x_{5}$ & $x_{4}$ & 0 & 0 & 0 & 0 & 0 & 1 \\
$x_{3}$ & 0 & 0 & 0 & $-x_{1}$ & 0 & 1 & 0 \\
0 & $x_{2}$ & 0 & $x_{1}$ & 0 & 1 & 0 & 0 \\
\hline 0 & 0 & 0 & 0 & 1 & 0 & 0 & 0 \\
0 & 0 & 0 & 1 & 0 & 0 & 0 & 0 \\
0 & 0 & 1 & 0 & 0 & 0 & 0 & 0 \\
0 & 1 & 0 & 0 & 0 & 0 & 0 & 0 \\
1 & 0 & 0 & 0 & 0 & 0 & 0 & 0 \\
\hline
\end{tabular}

FigURE 9.22.1

\begin{tabular}{|c|l|l|l|}
\hline & \multicolumn{1}{|c|}{$\operatorname{deg}$} & Weight & \multicolumn{1}{c|}{ Geometric condition } \\
\hline$F_{1}^{2}$ & $(1,0,1)_{P l}$ & $2 \pi_{3}$ & $\left\langle R_{5} \cap E_{7}+S_{5} \cap E_{7}\right\rangle \cap E_{3} \neq 0$ \\
$F_{1}$ & $(1,0,1)$ & $\omega_{3}$ & \\
\hline$F_{2}^{2}$ & $(1,2,0)_{P l}$ & $2 \pi_{1}+\pi_{5}^{\prime}$ & $\left\langle R_{5} \cap E_{9}+S_{2} \cap E_{9}\right\rangle \cap E_{5}^{\prime} \perp S_{2} \cap E_{9}$ \\
$F_{2}$ & $(1,1,0)$ & $\omega_{1}+\omega_{4}$ & \\
\hline$F_{3}$ & $(1,1,0)_{P l}$ & $\pi_{3}$ & $\left\langle R_{5}+S_{2}\right\rangle \cap E_{3} \neq 0$ \\
$F_{3}$ & $(2,1,0)$ & $\omega_{3}$ & \\
\hline$F_{4}^{2}$ & $(1,0,1)_{P l}$ & $2 \pi_{1}$ & $\left\langle R_{5} \cap E_{9}+S_{5} \cap E_{9}\right\rangle \cap E_{1} \neq 0$ \\
$F_{4}$ & $(1,0,1)$ & $\omega_{1}$ & \\
\hline$F_{5} F_{Q, 5}$ & $(1,1,1)_{P l}$ & $\pi_{2}+\pi_{4}$ & $\left\langle\left\langle R_{5} \cap E_{8}+S_{5} \cap E_{8}\right\rangle \cap E_{4}+S_{8} \cap E_{4}\right\rangle \cap E_{0} \neq 0$ \\
$F_{5}$ & $(2,1,1)$ & $\omega_{2}+\omega_{4}$ & \\
\hline$F_{6}^{2}$ & $(1,2,0)_{P l}$ & $\pi_{5}$ & $\left\langle\left\langle R_{5}+S_{2}\right\rangle \cap E_{5}+S_{8} \cap E_{5}\right\rangle \cap E_{0} \neq 0$ \\
$F_{6}$ & $(1,1,0)$ & $\omega_{5}$ & \\
\hline$F_{7}^{2}$ & $(1,2,1)_{P l}$ & $2 \pi_{3}$ & $\left\langle\left\langle\left\langle R_{5}+S_{2}\right\rangle \cap E_{7}+S_{5} \cap E_{7}\right\rangle \cap E_{3}+S_{8} \cap E_{3}\right\rangle \cap E_{0} \neq 0$ \\
$F_{7}$ & $(1,1,1)$ & $\omega_{3}$ & \\
\hline$F_{1} F_{6}$ & & & \\
$F_{3} F_{Q, 5}$ & $(2,1,1)$ & $\omega_{3}+\omega_{5}$ & \\
$F_{7} F_{P, 5}$ & & & \\
\hline
\end{tabular}

Let us explain why the form $f_{5}$ corresponding to the geometric condition $\left\langle\left\langle R_{5} \cap E_{8}+\right.\right.$ $\left.\left.S_{5} \cap E_{8}\right\rangle \cap E_{4}+S_{8} \cap E_{4}\right\rangle \cap E_{0} \neq 0$ specifies the divisor $D_{5}+D_{Q, 5}$. First, the form $F_{5}$ should depend on the Plücker coordinates of all three spaces, because the divisors depending on the coordinates of fewer subspaces are known from cases 1 and 4, with the preimages of the divisors in these cases (except for the Schubert divisors) under 
the canonical projection being $D_{1}, D_{4}$ and $D_{2}, D_{3}, D_{6}$, respectively. Let us show that the form $f_{5}$ is divisible by $F_{Q, 5}$. Then the quotient will be equal to $F_{5}$. Indeed, the multidegree and the weight of the quotient are equal to $(2,1,1)$ and $\omega_{2}+\omega_{4}$, the quotient is not divisible by $F_{P, 5}$ by the weight argument, and the multidegree of $F_{5}$ should not have zero components.

\begin{tabular}{|cc|ccc|ccc|}
\hline$*$ & $*$ & $*$ & $*$ & $*$ & $*$ & $*$ & $*$ \\
$*$ & $*$ & $*$ & $*$ & $*$ & $*$ & $*$ & $*$ \\
$*$ & $*$ & $*$ & $*$ & $*$ & $*$ & $*$ & 1 \\
$*$ & $*$ & $*$ & 0 & 1 & 0 & 0 & 0 \\
$*$ & $*$ & $*$ & 1 & 0 & 0 & 0 & 0 \\
\hline$*$ & $*$ & $*$ & 0 & $-x$ & 0 & 1 & 0 \\
$*$ & $*$ & $*$ & $x$ & 0 & 1 & 0 & 0 \\
$*$ & $*$ & 1 & 0 & 0 & 0 & 0 & 0 \\
0 & 1 & 0 & 0 & 0 & 0 & 0 & 0 \\
1 & 0 & 0 & 0 & 0 & 0 & 0 & 0 \\
\hline
\end{tabular}

Figure 9.22.2

We use the action of the group $H_{1}$ as in Figure $9.1 .2 \mathrm{a}$ in case 1 ; then $R_{5}$ can be reduced to the canonical form. (We assume that the point does not lie in $D_{P, 5}$.) In the chart $s_{10,9,8,5,4} \neq 0$, the subspaces $S_{2}, S_{5}$, and $S_{8}$ can be reduced to the form shown in Figure 9.22.2 The expression $\left\langle R_{5} \cap E_{8}+S_{5} \cap E_{8}\right\rangle \cap E_{4}$ determines the subspace spanned by the first two vectors highlighted in gray. If the point lies in $D_{Q, 5}$, then the expression $S_{8} \cap E_{4}$ determines the subspace spanned by the second and third vectors highlighted in gray. The determinant of the matrix formed by the basis vectors of these two subspaces is zero. It follows that the form is zero at the points of the divisor $D_{Q, 5}$.

23. $(5,5),(2,3,3,2)^{\prime}$.

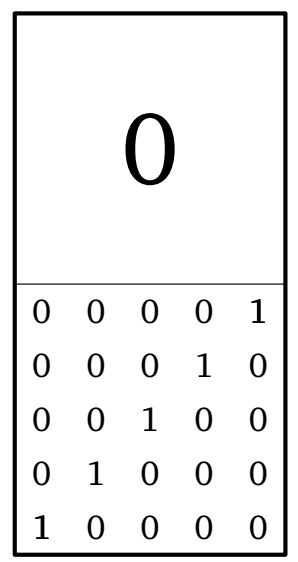

\begin{tabular}{|cc|ccc|ccc|}
\hline 0 & $-x_{6}$ & $-x_{5}$ & $-x_{2}$ & 0 & 0 & 0 & 0 \\
$x_{6}$ & 0 & 0 & $-x_{3}$ & 0 & $-x_{1}$ & 0 & 0 \\
$x_{5}$ & 0 & 0 & $-x_{4}$ & 0 & 0 & 0 & 1 \\
$x_{2}$ & $x_{3}$ & $x_{4}$ & 0 & 0 & 0 & 1 & 0 \\
0 & $x_{1}$ & 0 & 0 & 1 & 0 & 0 & 0 \\
\hline 0 & 0 & 0 & 0 & 0 & 1 & 0 & 0 \\
0 & 0 & 0 & 1 & 0 & 0 & 0 & 0 \\
0 & 0 & 1 & 0 & 0 & 0 & 0 & 0 \\
0 & 1 & 0 & 0 & 0 & 0 & 0 & 0 \\
1 & 0 & 0 & 0 & 0 & 0 & 0 & 0 \\
\hline
\end{tabular}

FiguRe 9.23 


\begin{tabular}{|c|l|l|l|}
\hline & \multicolumn{1}{|c|}{ deg } & \multicolumn{1}{c|}{ Weight } & \multicolumn{1}{|c|}{ Geometric condition } \\
\hline$F_{1}^{2}$ & $(1,2,0)_{P l}$ & $2 \pi_{1}+\pi_{5}^{\prime}$ & $\left\langle R_{5} \cap E_{9}+S_{2} \cap E_{9}\right\rangle \cap E_{5}^{\prime} \perp S_{2} \cap E_{9}$ \\
$F_{1}$ & $(1,1,0)$ & $\omega_{1}+\omega_{4}$ & \\
\hline$F_{2}$ & $(1,1,0)_{P l}$ & $\pi_{3}$ & $\left\langle R_{5}+S_{2}\right\rangle \cap E_{3} \neq 0$ \\
$F_{2}$ & $(2,1,0)$ & $\omega_{3}$ & \\
\hline$F_{3}^{2}$ & $(1,2,1)_{P l}$ & $2 \pi_{1}+2 \pi_{3}$ & $\left\langle\left\langle\left\langle R_{5} \cap E_{9}+S_{2} \cap E_{9}\right\rangle \cap E_{7}\right.\right.$ \\
$F_{3}$ & $(1,1,1)$ & $\omega_{1}+\omega_{3}$ & $\left.\left.+S_{5}^{\prime} \cap E_{7}\right\rangle \cap E_{3}+S_{8} \cap E_{3}\right\rangle \cap E_{1} \neq 0$ \\
\hline$F_{4}^{2}$ & $(1,0,1)_{P l}$ & $2 \pi_{2}$ & $\left\langle R_{5} \cap E_{8}+S_{5}^{\prime} \cap E_{8}\right\rangle \cap E_{2} \neq 0$ \\
$F_{4}$ & $(1,0,1)$ & $\omega_{2}$ & \\
\hline$F_{6}^{2}$ & $(1,2,0)_{P l}$ & $\pi_{5}$ & $\left\langle\left\langle R_{5}+S_{2}\right\rangle \cap E_{5}+S_{8} \cap E_{5}\right\rangle \cap E_{0} \neq 0$ \\
$F_{6}$ & $(1,1,0)$ & $\omega_{5}$ & \\
\hline$F_{7}^{2}$ & $(1,0,1)_{P l}$ & 0 & $\left\langle R_{5}+S_{5}^{\prime}\right\rangle \cap E_{0} \neq 0$ \\
$F_{7}$ & $(1,0,1)$ & 0 & \\
\hline$F_{4} F_{6}$ & $(2,1,1)$ & $\omega_{2}+\omega_{5}$ & \\
$F_{7} F_{P, 5} F_{Q, 2}$ & & & \\
\hline & & & \\
\hline
\end{tabular}

There also exists a parametric divisor $D_{5}$ such that the form $F_{5} F_{Q, 5}^{\prime}$ is given by the geometric condition

$$
\left\langle\left\langle R_{5} \cap E_{8}+S_{5}^{\prime} \cap E_{8}\right\rangle \cap E_{4}+S_{8} \cap E_{4}\right\rangle \cap E_{0} \neq 0 .
$$

The explanation of the fact that this geometric condition specifies the divisor $D_{5}+D_{Q, 5}^{\prime}$ is similar to the preceding case.

24. $(5,5),(3,2,2,3)$.

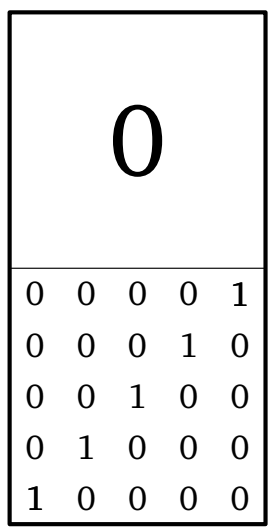

\begin{tabular}{|ccc|cc|cc|}
\hline 0 & $-x_{6}$ & $-x_{5}$ & 0 & 0 & 0 & 0 \\
$x_{6}$ & 0 & $-x_{4}$ & $-x_{3}$ & 0 & 0 & 0 \\
$x_{5}$ & $x_{4}$ & 0 & 0 & $-x_{2}$ & 0 & 0 \\
0 & $x_{3}$ & 0 & 0 & $-x_{1}$ & 0 & 1 \\
0 & 0 & $x_{2}$ & $x_{1}$ & 0 & 1 & 0 \\
\hline 0 & 0 & 0 & 0 & 1 & 0 & 0 \\
0 & 0 & 0 & 1 & 0 & 0 & 0 \\
0 & 0 & 1 & 0 & 0 & 0 & 0 \\
0 & 1 & 0 & 0 & 0 & 0 & 0 \\
1 & 0 & 0 & 0 & 0 & 0 & 0 \\
\hline
\end{tabular}

FiguRE 9.24 


\begin{tabular}{|c|l|l|l|}
\hline & \multicolumn{1}{|c|}{ deg } & \multicolumn{1}{|c|}{ Weight } & \multicolumn{1}{c|}{ Geometric condition } \\
\hline$F_{1}^{2}$ & $(1,0,1)_{P l}$ & $2 \pi_{3}$ & $\left\langle R_{5} \cap E_{7}+S_{5} \cap E_{7}\right\rangle \cap E_{3} \neq 0$ \\
$F_{1}$ & $(1,0,1)$ & $\omega_{3}$ & \\
\hline$F_{2}^{2}$ & $(1,2,0)_{P l}$ & $2 \pi_{2}+\pi_{5}^{\prime}$ & $\left\langle R_{5} \cap E_{8}+S_{3} \cap E_{8}\right\rangle \cap E_{5}^{\prime} \perp S_{3} \cap E_{8}$ \\
$F_{2}$ & $(1,1,0)$ & $\omega_{2}+\omega_{4}$ & \\
\hline$F_{3}$ & $(1,1,0)_{P l}$ & $\pi_{1}+\pi_{3}$ & $\left\langle R_{5} \cap E_{9}+S_{3} \cap E_{9}\right\rangle \cap E_{3} \neq 0$ \\
$F_{3}$ & $(2,1,0)$ & $\omega_{1}+\omega_{3}$ & \\
\hline$F_{4}^{2}$ & $(1,2,0)_{P l}$ & $2 \pi_{1}+\pi_{5}$ & $\left\langle\left\langle R_{5} \cap E_{9}+S_{3} \cap E_{9}\right\rangle \cap E_{5}+S_{7} \cap E_{5}\right\rangle \cap E_{1} \neq 0$ \\
$F_{4}$ & $(1,1,0)$ & $\omega_{1}+\omega_{5}$ & \\
\hline$F_{5}$ & $(1,1,0)_{P l}$ & $\pi_{2}$ & $\left\langle R_{5}+S_{3}\right\rangle \cap E_{2} \neq 0$ \\
$F_{5}$ & $(2,1,0)$ & $\omega_{2}$ & \\
\hline$F_{6}^{2}$ & $(1,2,0)_{P l}$ & $\pi_{5}^{\prime}$ & $\left\langle\left\langle R_{5}+S_{3}\right\rangle \cap E_{5}^{\prime}+S_{7} \cap E_{5}^{\prime}\right\rangle \cap E_{0} \neq 0$ \\
$F_{6}$ & $(1,1,0)$ & $\omega_{4}$ & \\
\hline$F_{7}^{2}$ & $(1,0,1)_{P l}$ & $2 \pi_{1}$ & $\left\langle R_{5} \cap E_{9}+S_{5} \cap E_{9}\right\rangle \cap E_{1} \neq 0$ \\
$F_{7}$ & $(1,0,1)$ & $\omega_{1}$ & \\
\hline$F_{1} F_{4}$ & & & \\
$F_{3} F_{Q, 5}$ & $(2,1,1)$ & $\omega_{1}+\omega_{3}+\omega_{5}$ & \\
$F_{7} F_{Q, 3} F_{P, 5}$ & & & \\
\hline
\end{tabular}


25. $(5,5),(3,2,2,3)^{\prime}$.

\begin{tabular}{|c|l|l|l|}
\hline & \multicolumn{1}{|c|}{ deg } & Weight & \multicolumn{1}{c|}{ Geometric condition } \\
\hline$F_{1}^{2}$ & $(1,2,0)_{P l}$ & $2 \pi_{2}+\pi_{5}^{\prime}$ & $\left\langle R_{5} \cap E_{8}+S_{3} \cap E_{8}\right\rangle \cap E_{5}^{\prime} \perp S_{3} \cap E_{8}$ \\
$F_{1}$ & $(1,1,0)$ & $\omega_{2}+\omega_{4}$ & \\
\hline$F_{2}^{2}$ & $(1,0,1)_{P l}$ & $2 \pi_{2}$ & $\left\langle R_{5} \cap E_{8}+S_{5}^{\prime} \cap E_{8}\right\rangle \cap E_{2} \neq 0$ \\
$F_{2}$ & $(1,0,1)$ & $\omega_{2}$ & \\
\hline$F_{3}$ & $(1,1,0)_{P l}$ & $\pi_{1}+\pi_{3}$ & $\left\langle R_{5} \cap E_{9}+S_{3} \cap E_{9}\right\rangle \cap E_{3} \neq 0$ \\
$F_{3}$ & $(2,1,0)$ & $\omega_{1}+\omega_{3}$ & \\
\hline$F_{4}^{2}$ & $(1,2,0)_{P l}$ & $2 \pi_{1}+\pi_{5}$ & $\left\langle\left\langle R_{5} \cap E_{9}+S_{3} \cap E_{9}\right\rangle \cap E_{5}+S_{7} \cap E_{5}\right\rangle \cap E_{1} \neq 0$ \\
$F_{4}$ & $(1,1,0)$ & $\omega_{1}+\omega_{5}$ & \\
\hline$F_{5}$ & $(1,1,0)_{P l}$ & $\pi_{2}$ & $\left\langle R_{5}+S_{3}\right\rangle \cap E_{2} \neq 0$ \\
\hline$F_{5}$ & $(2,1,0)$ & $\omega_{2}$ & \\
\hline$F_{6}^{2}$ & $(1,2,0)_{P l}$ & $\pi_{5}^{\prime}$ & $\left\langle\left\langle R_{5}+S_{3}\right\rangle \cap E_{5}^{\prime}+S_{7} \cap E_{5}^{\prime}\right\rangle \cap E_{0} \neq 0$ \\
\hline$F_{6}$ & $(1,1,0)$ & $\omega_{4}$ & \\
\hline$F_{7}^{2}$ & $(1,0,1)_{P l}$ & 0 & $\left\langle R_{5}+S_{5}^{\prime}\right\rangle \cap E_{0} \neq 0$ \\
\hline$F_{7}$ & $(1,0,1)$ & 0 & \\
\hline$F_{2} F_{6}$ & $(2,1,1)$ & $\omega_{2}+\omega_{4}$ & \\
\hline$F_{1} F_{7}$ & & & \\
\hline
\end{tabular}

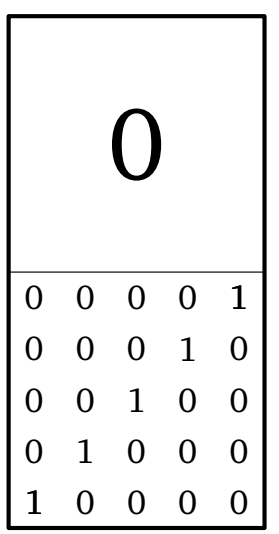

\begin{tabular}{|ccc|cc|cc|}
\hline 0 & $-x_{6}$ & $-x_{5}$ & 0 & 0 & 0 & 0 \\
$x_{6}$ & 0 & $-x_{4}$ & $-x_{3}$ & 0 & 0 & 0 \\
$x_{5}$ & $x_{4}$ & 0 & $-x_{2}$ & 0 & $-x_{1}$ & 0 \\
0 & $x_{3}$ & $x_{2}$ & 0 & 0 & 0 & 1 \\
0 & 0 & $x_{1}$ & 0 & 1 & 0 & 0 \\
\hline 0 & 0 & 0 & 0 & 0 & 1 & 0 \\
0 & 0 & 0 & 1 & 0 & 0 & 0 \\
0 & 0 & 1 & 0 & 0 & 0 & 0 \\
0 & 1 & 0 & 0 & 0 & 0 & 0 \\
1 & 0 & 0 & 0 & 0 & 0 & 0 \\
\hline
\end{tabular}

FiguRe 9.25 
26. $(6,6),(4,4,4)$

\begin{tabular}{|c|l|l|l|}
\hline & \multicolumn{1}{|c|}{ deg } & Weight & \multicolumn{1}{c|}{ Geometric condition } \\
\hline$F_{1}^{2}$ & $(1,2,0)_{P l}$ & $2 \pi_{3}+\pi_{6}^{\prime}$ & $\left\langle R_{6} \cap E_{9}+S_{4} \cap E_{9}\right\rangle \cap E_{6}^{\prime} \perp S_{4} \cap E_{9}$ \\
$F_{1}$ & $(1,1)$ & $\omega_{3}+\omega_{5}$ & \\
\hline$F_{2}$ & $(1,1,0)_{P l}$ & $\pi_{2}+\pi_{4}$ & $\left\langle R_{6} \cap E_{10}+S_{4} \cap E_{10}\right\rangle \cap E_{4} \neq 0$ \\
$F_{2}$ & $(2,1)$ & $\omega_{2}+\omega_{4}$ & \\
\hline$F_{3}^{3}$ & $(1,2,0)_{P l}$ & $2 \pi_{2}+\pi_{6}$ & $\left\langle\left\langle R_{6} \cap E_{10}+S_{4} \cap E_{10}\right\rangle \cap E_{6}+S_{8} \cap E_{6}\right\rangle \cap E_{2} \neq 0$ \\
$F_{3}$ & $(1,1)$ & $\omega_{2}+\omega_{6}$ & \\
\hline$F_{4}$ & $(1,1,0)_{P l}$ & $\pi_{1}+\pi_{3}$ & $\left\langle R_{6} \cap E_{11}+S_{4} \cap E_{11}\right\rangle \cap E_{3} \neq 0$ \\
$F_{4}$ & $(2,1)$ & $\omega_{1}+\omega_{3}$ & \\
\hline$F_{5}$ & $(1,1,0)_{P l}$ & $\pi_{2}$ & $\left\langle R_{6}+S_{4}\right\rangle \cap E_{2} \neq 0$ \\
$F_{5}$ & $(2,1)$ & $\omega_{2}$ & \\
\hline$F_{6}^{2}$ & $(1,2,0)_{P l}$ & $2 \pi_{1}+\pi_{6}^{\prime}$ & $\left\langle\left\langle R_{6} \cap E_{11}+S_{4} \cap E_{11}\right\rangle \cap E_{6}^{\prime}+S_{8} \cap E_{6}^{\prime}\right\rangle \cap E_{1} \neq 0$ \\
$F_{6}$ & $(1,1)$ & $\omega_{1}+\omega_{5}$ & \\
\hline$F_{7}^{2}$ & $(2,2,0)_{P l}$ & $2 \pi_{4}$ & $\left\langle\left\langle R_{6}+S_{4}\right\rangle \cap E_{4}+\left\langle R_{6} \cap E_{8}+S_{8} \cap E_{8}\right\rangle \cap E_{4}\right\rangle \cap E_{0} \neq 0$ \\
$F_{7}$ & $(2,1)$ & $\omega_{4}$ & \\
\hline$F_{8}^{2}$ & $(1,2,0)_{P l}$ & $\pi_{6}$ & $\left\langle\left\langle R_{6}+S_{4}\right\rangle \cap E_{6}+S_{8} \cap E_{6}\right\rangle \cap E_{0} \neq 0$ \\
$F_{8}$ & $(1,1)$ & $\omega_{6}$ & \\
\hline$F_{3} F_{7}$ & & & \\
$F_{5} F_{P, 6} F_{Q, 4}$ & $(3,2)$ & $\omega_{2}+\omega_{4}$ & \\
$F_{2} F_{8}$ & & & \\
\hline
\end{tabular}

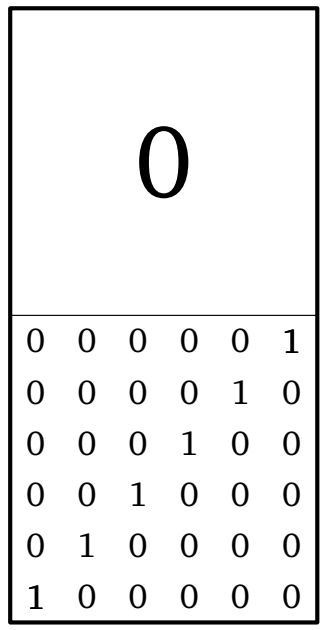

\begin{tabular}{|cccc|cccc|}
\hline 0 & $-x_{7}$ & $-x_{5}$ & 0 & 0 & 0 & 0 & 0 \\
$x_{7}$ & 0 & $-x_{6}$ & $-x_{4}$ & 0 & 0 & 0 & 0 \\
$x_{5}$ & $x_{6}$ & 0 & $-x_{3}$ & $-x_{2}$ & 0 & 0 & 0 \\
0 & $x_{4}$ & $x_{3}$ & 0 & 0 & $-x_{1}$ & 0 & 0 \\
0 & 0 & $x_{2}$ & 0 & 0 & 0 & 0 & 1 \\
0 & 0 & 0 & $x_{1}$ & 0 & 0 & 1 & 0 \\
\hline 0 & 0 & 0 & 0 & 0 & 1 & 0 & 0 \\
0 & 0 & 0 & 0 & 1 & 0 & 0 & 0 \\
0 & 0 & 0 & 1 & 0 & 0 & 0 & 0 \\
0 & 0 & 1 & 0 & 0 & 0 & 0 & 0 \\
0 & 1 & 0 & 0 & 0 & 0 & 0 & 0 \\
1 & 0 & 0 & 0 & 0 & 0 & 0 & 0 \\
\hline
\end{tabular}

Figure 9.26 
The remaining cases can be omitted, because $\mathrm{SO}_{6}$ and $\mathrm{SO}_{5}$ are locally isomorphic to $\mathrm{SL}_{4}$ and $\mathrm{Sp}_{4}$, respectively.

\section{REFERENCES}

[1] É. B. Vinberg and A. L. Onishchik, A seminar on Lie groups and algebraic groups, Nauka, Moscow, 1988. (Russian) MR 1090326(92i:22014)

[2] J. Hausen, Three lectures on Cox rings, LMS Lecture Note Series 405 (2013), 3-60. MR.3077165

[3] P. Littelmann, On spherical double cones, J. Algebra 166 (1994), no. 1, 142-157. MR1276821 (95c:14066)

[4] D. I. Panyushev, Complexity and rank of actions in invariant theory, J. Math. Sci. 95 (1999), no. 1, 1925-1985. MR.1708594 (2000h:14041)

[5] E. V. Ponomareva, Classification of double flag varieties of complexity 0 and 1, Izv. Ross. Akad. Nauk Ser. Mat. 77 (2013), no. 5, 155-178; English transl., Izv. Math. 77 (2013), no. 5, 998-1020. MR 3137198

[6] J. Stembridge, Multiplicity-free products and restrictions of Weyl characters, Represent. Theory. 7 (2003), 404-439. MR2017064 (2004j:17013)

[7] D. A. Timashev, Homogeneous spaces and equivariant embeddings, Encyclopedia of Math. Sci., vol. 138, Springer, 2011. MR2797018(2012e:14100)

Faculty of Mechanics and Mathematics, Lomonosov Moscow State University, Moscow, Russia

E-mail address: lizaveta@yandex.ru

Translated by V. E. NAZAIKINSKII

Originally published in Russian 